Published in final edited form as:

Curr Probl Surg. 2018 June ; 55(6): 198-246. doi:10.1067/j.cpsurg.2018.07.001.

\title{
Robotic gastrointestinal surgery
}

Seth I. Felder, MD\#a, Rajesh Ramanathan, MD\#b, Ashley E. Russo, MD" ${ }^{\# c}$, Rosa Maria Jimenez-Rodriguez, MD, PhD' ${ }^{\mathrm{c}}$, Melissa E. Hogg, MD, MS ${ }^{\mathrm{b}}$, Amer H. Zureikat, MD ${ }^{\mathrm{b}}$, Vivian E. Strong, $\mathbf{M D}^{\mathrm{c}}$, Herbert J. Zeh, $\mathbf{M D}^{\mathrm{b}}$, and Martin R. Weiser, $\mathbf{M D}^{\mathrm{c},{ }^{*}}$

aDepartment of Gastrointestinal Surgery, Moffitt Cancer Center, Tampa, Florida

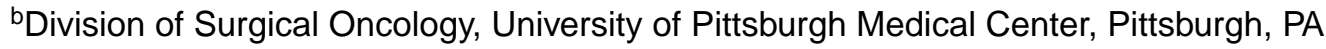

'Department of Surgery, Memorial Sloan Kettering Cancer Center, New York, NY

\# These authors contributed equally to this work.

\section{Robotic colorectal surgery}

Seth I. Felder, Rosa Maria Jimenez-Rodriguez, and Martin R. Weiser

Robotic surgery builds on the innovations of laparoscopy, which has been increasingly adopted for colon and rectal cancer surgery over the last 2 decades following numerous welldesigned trials that demonstrated equivalent oncological outcomes to those of open surgery. These trials also showed that laparoscopic approaches shorten recovery and reduce complication rates, and favorable results have remained valid in long-term follow-up. Another advantage of minimally invasive techniques for resecting colon and rectal cancer is improved visualization of the surgical field, translating into greater operative exposure, exploiting 1 of surgery's most fundamental tenets.

Wider adoption of laparoscopic colorectal cancer resection has been hindered by its technological constraints and the advanced skill set it requires. Because exposure, tension, and dissection are translated through long, rigid, and nonarticulating instruments, dexterity is reduced due to the fulcrum effect, involuntary motions are magnified, and ergonomics are suboptimal. These mechanical limitations are particularly problematic for rectal cancer, where the pelvis presents anatomical constraints. Further, laparoscopy compromises operative control, as the surgeon must choose between operating with 2 instruments while an assistant positions the camera, or manipulating the camera and using only 1 laparoscopic instrument, which also requires expert assistance.

Advantages of robotics over laparoscopy-Robotic surgery resolves many of the mechanical and optical disadvantages of laparoscopy. It further magnifies the view of the operative field, refines the instrumentation to achieve exposure and retraction, and allows the primary surgeon enhanced control by reducing reliance on a secondary expert surgeon or assistant.

*Corresponding author: Martin R. Weiser, MD, Department of Surgery, Memorial Sloan Kettering Cancer Center, 1275 York Avenue, New York, NY 10065., weiser1@ mskcc.org (M.R. Weiser). 
Robotic surgical equipment allows flexible, wristed movement capabilities, self-assistance through a third robotic arm, and high-definition 3-dimensional views from a mounted, stabilized surgeon-controlled camera. ${ }^{1,2}$ The superior ergonomics and surgical dexterity provided by the robot result from the instruments' $7^{\circ}$ of freedom, $90^{\circ}$ articulation, and $540^{\circ}$ rotation, permitting manipulation within small spaces, which is particularly advantageous in the narrow, bony pelvis. ${ }^{3-5}$ Compared to laparoscopy, the robot has been shown to enhance dexterity by $65 \%$, reduce skill-based errors by $93 \%$, and shorten the time needed to complete a task by $40 \% .{ }^{6}$ Robotic technology also provides motion scaling and tremor filtering, facilitating precise dissection and suturing, which is particularly valuable in dissecting along the origins of the mesenteric vessels during complete mesocolic excision or in performing total mesorectal excision (TME) within the pelvis. ${ }^{7}$ Additionally, the robotic platform enables an integrated and supervised teaching environment without compromising operative or long-term outcomes. ${ }^{4}$

Adoption of robotics for colorectal surgery-Robotic colorectal surgery was first described in 2001, and the first robotic TME was reported in 2006, ${ }^{4,8-11}$ but adoption of robotics into the colon and rectal surgical armamentarium has been relatively slow; it is used in only $2.8 \%$ of minimally invasive colorectal surgery. ${ }^{12,13}$ A major reason for this low rate is the expense of robotic surgical systems, which require an initial investment ranging from $\$ 1$ to $\$ 2.5$ million for each unit, ${ }^{14-16}$ as well as ongoing maintenance and repair, costly consumables, and additional team training.

Robotics has been found to increase the cost of colorectal cancer surgery by approximately $\$ 2000$ to 5000 per procedure compared to laparoscopy. ${ }^{17-23}$ However, robotic surgery can be cost-effective when performed by high-volume surgeons at high-volume centers, ${ }^{24}$ although the number of resections that must be performed at an institution to make robotic costs manageable has yet to be determined. ${ }^{4}$ The ROLARR (Robotic versus Laparoscopic Resection for Rectal Cancer) trial found an additional cost of only $\$ 1100$ despite using the older da Vinci Si system, which had limited multiquadrant technologic capabilities, resulting in a large proportion of hybrid laparoscopic-robotic operations due to learning-curve challenges. This suggests that costs will likely decrease with newer robotic iterations that reduce docking and positioning challenges.

Another contributor to slow adoption of robotics for colorectal surgery is the scarcity of high-level evidence of its superiority over laparoscopy. A large randomized controlled study, ROLARR, ${ }^{25,26}$ demonstrated their equivalence for rectal cancer surgery, and 2 other randomized studies, RLOAPR ${ }^{27}$ and COLRAR (Comparison of Laparoscopic vs. Robotassisted Surgery for Rectal Cancer), ${ }^{28}$ are in progress. The majority of reports on robotic surgery outcomes derive from single-institution studies or database audits, ${ }^{29-35}$ which also show that oncologic and recovery outcomes are at least comparable to those of laparoscopic colorectal procedures. One study suggests that the robotic approach may prove superior to laparoscopy in conversion rates and, conceivably, oncologic outcomes. ${ }^{36}$

Further limiting the widespread use of robotics for colorectal procedures is the extended learning curve. Perhaps in part because of the lack of haptic feedback and/or tactile sensation, training an individual surgeon requires more than 70 cases. However, this time has 
been reported to shorten once a program and its infrastructure has been established at a particular institution (which requires approximately 25 cases). This acceleration is attributable to standard positioning of ports, patients, and the robotic system, as well as a trained surgical team with clearly established roles (nurses, bedside assistants, equipment managers, and scrub technicians). ${ }^{37}$

The relationship between increasing surgeon volume and better outcomes of robotic surgery has been well documented. A recent study evaluating 17,749 patients undergoing colon cancer resection between 2008 and 2011 showed that, compared to low-volume surgeons ( $<5$ procedures per year), colectomy performed by high-volume surgeons $(>11$ procedures per year) cost $\$ 1500$ less, with 25\% fewer complications and a 30\% lower reoperation rate. ${ }^{38}$ Similar improvements in outcomes and reductions in cost with increasing procedure volume for both surgeons and hospitals have been demonstrated for robotic colorectal surgery. ${ }^{2,22,39,40}$ For comparative context, more than 20 laparoscopic proctectomy procedures are required to gain proficiency in that procedure, as surgeon skill (measured by margin positivity rate) increased over the course of the CLASICC (Conventional versus Laparoscopic-assisted Surgery in Colorectal Cancer) trial (20 past cases was the minimum for surgeon participation). ${ }^{41}$

Evidence supporting the use of robotic colectomy-The appeal of robotics for colectomy is the potential to enhance the use of minimally invasive techniques for these procedures. Despite validation of the advantages of laparoscopy for colectomy, its use remains fairly low, at approximately 55\% of colectomies performed in the United States. 42-44 When applying the principles of TME to colon cancer surgery, with meticulous dissection in the mesocolic plane and central mesenteric vessel ligation, the technical difficulties of laparoscopy are compounded..$^{45-47}$ The most likely explanation for this limited use is surgeons' lack of technical proficiency, or lack of confidence; many surgeons consider open colectomy as more likely to produce an oncologically sound outcome. ${ }^{43}$

Wide adoption of robotic colectomy requires evidence of its safety, feasibility, and equivalence or superiority to existing approaches in terms of outcomes, and numerous such reports have been published in support of robotic right colectomy. ${ }^{35,48-56}$ The strongest of these is a randomized controlled trial comparing robotic and laparoscopic right hemicolectomy. Park and colleagues reported no significant difference in estimated blood loss, conversion rate, length of stay, surgical complications, postoperative pain, resection margins, or lymph node clearance among the 70 patients studied ${ }^{53}$ However, the robotic approach was associated with a significantly longer operative time (195 vs 130 minutes; $P<$ 0.001 ), which may relate to the greater frequency of intracorporeal anastomosis in those procedures, and an approximately $16 \%$ higher total hospital cost $(\$ 12,235$ vs $\$ 10,319 ; P=$ 0.013). Similarly, a retrospective study of right colectomy at a single institution by deSouza and colleagues found no difference in conversion rate, resection margin positivity, lymph node yield, length of stay, or morbidity between robotic $(n=40)$ and laparoscopic $(n=135)$ procedures. ${ }^{51,57}$ The robotic approach was associated with significantly longer operative times (mean, 159 vs 118 minutes; $P<0.001$ ) and significantly higher total cost (median, $\$ 15,192$ vs $\$ 12,362 ; P=0.003)$. 
Other studies have found slight advantages for robotics in right colectomy. In a casematched comparison of robotic and open surgery for right-sided colon cancers, Luca and colleagues found that robotic surgery was associated with shorter hospital stays ( 5 vs 8 days; $P=0.001)$ and resection of at least 15 lymph nodes $(100 \%$ vs $88.2 \% ; P=0.038) .56,57$ The mean operative time was significantly longer in the robotic group (192 vs 136 minutes; $P<$ 0.001 ). More conclusively, a meta-analysis of 7 studies (including the randomized trial by Park and colleagues $),{ }^{48}$ found that in right colectomy, use of robotics was associated with lower estimated blood loss $(P=0.0002)$, fewer postoperative complications $(P=0.02)$, and significantly faster recovery of bowel function $(P<0.00001)$ compared with laparoscopy. There were no differences in the length of hospital stay $(P=0.12)$, rate of conversion to open surgery $(P=0.48)$, anastomotic leak $(P=0.28)$, or bleeding $(P=0.95)$, although operative time was longer $(P<0.00001)$.

There are additional, more recent, data to indicate that robotic surgery is associated with fewer conversions from minimally invasive to open colorectal surgery than traditional laparoscopy. As conversion increases wound infection and complication rates and length of stay, and may impact cancer-specific outcomes, ${ }^{58-61}$ this lower rate represents a key advantage. An analysis of the Michigan Surgical Quality Collaborative Database found conversion rates of $9 \%$ and $16.9 \%$ for robotic and laparoscopic colectomy, respectively $(P=$ 0.06), corresponding to a 1.6- to 1.8-day shorter stay for robotic surgery. ${ }^{39}$ For rectal resections, the difference in conversion rates reached statistical significance $(21.2 \%$ vs $7.8 \%$, respectively; $P<0.001)$. Using the National Inpatient Database, a matched analysis found significantly lower conversion rates for robotic compared with laparoscopic colectomy (5.7\% vs 9.9\%, respectively; $P=0.05) .{ }^{18}$ A recent meta-analysis found that 10 of 22 studies with laparoscopy as a control group reported lower conversion rates from robotic to open surgery, with 3 studies reporting statistically significant differences. ${ }^{62}$

Because robotics simplifies complex surgical maneuvers, it would likely facilitate intracorporeal anastomoses which may have advantages in short-term and long-term recovery. By limiting visceral stretch and trauma associated with extraction and extracorporeal anastomosis, intracorporeal anastomosis may accelerate recovery. ${ }^{63-65}$ Intracorporeal anastomoses are also associated with a lower rate of surgical site infections and incisional hernias than those created extracorporeally. ${ }^{4,66}$ By expanding possible sites of specimen extraction, intracorporeal anastomosis allows the surgeon to extract the specimen through a Pfannenstiel incision. Lee and colleagues reported an incisional hernia incidence of $29 \%$ compared to $0 \%$ when a Pfannenstiel extraction site was used. ${ }^{67}$ Similarly, the group at Memorial Sloan Kettering Cancer Center reported incisional hernia rates of 22\% and 17\% for laparoscopic and robotic midline right colectomy specimen extraction, respectively. No incisional hernia in the Pfannenstiel incision group was diagnosed, although that group included only 6 patients. ${ }^{68}$ The combined data suggest a potential advantage of off-midline incision, making intracorporeal anastomosis appealing.

Meta-analyses comparing intracorporeal and extracorporeal anastomoses have demonstrated similar leak rates but fewer wound infections and shorter length of stay for intracorporeal anastomosis. ${ }^{63,64,69}$ Although intracorporeal anastomosis is technically more challenging and may potentially lengthen operating time, the robotic platform facilitates exposure and 
suturing. ${ }^{70}$ Indeed, a retrospective study by Trastulli and colleagues found that robotic colectomy with intracorporeal anastomosis resulted in a shorter length of stay than laparoscopic right hemicolectomy with extracorporeal anastomosis. ${ }^{35}$ Both groups had similar patient demographics, pathology characteristics, and postoperative complication rates.

Multiple reports have also demonstrated that robotic left colectomy and sigmoid resection are technically feasible and safe. ${ }^{49,55,71}$ Although short-term oncological and postoperative outcomes of robotic colon resection have been reported in numerous studies, well-designed, prospective comparative studies are needed to determine long-term oncologic outcomes. ${ }^{4}$

On the basis of the limited available data, it appears that robotic colectomy is both feasible and safe but is associated with increased costs and longer operative times compared with laparoscopic or open approaches. It is not clear how the learning curve, type of anastomosis, and version of robotic platform affects these factors. In addition, hospital cost is subject to large variation depending on the surgeon's operative preferences and the logistics of individual health-care systems. Experienced robotic surgeons argue that operative times are not longer than those for conventional laparoscopy, as docking times can be shorter than 5 minutes, ${ }^{72}$ and the cost of robotic instruments can be comparable to that of the expensive energy devices routinely used in laparoscopic surgery. The main benefits arising from robotic colectomy compared to laparoscopy are likely to be oncologic, particularly when a complete mesocolic excision is performed, due to the improved accuracy of dissection. However, these differences will be subtle and will not be apparent in small series with only short-term follow-up. ${ }^{57}$

Contraindications to robotics-There are no absolute contraindications to robotic colon and rectal cancer surgery, and its application is limited primarily by the surgeon's experience and expertise. Relative contraindications, depending on the surgeon's judgment, are locally invasive tumors and recurrent disease, which often obscure normal anatomic planes.

Robotic right colectomy technique-Several techniques have been described for robotic right colectomy. The principal differences relate to port placement, operative approach (medial to lateral or lateral to medial), and whether an extracorporeal or intracorporeal anastomosis is undertaken. A total robotic approach maximizes the benefits of the technology.

The patient is placed supine on the operating table. The arms are secured by the patient's side, pressure points are protected with padding, and the patient is confirmed to be secure on the table. A Veress needle inserted below the left subcostal margin in the midclavicular line is the preferred method of establishing pneumoperitoneum because it allows rapid access and is appropriate for all patients. The first $8 \mathrm{~mm}$ robotic trocar is placed superior to the umbilicus. Three additional robotic $8 \mathrm{~mm}$ ports are placed: left upper quadrant, midline subumbilical, and right lower quadrant. An assistant port for pneumoperitoneum or AirSeal device is placed in the left lateral position (Fig. 1). The patient is then placed in a slight Trendelenburg position, with an $8^{\circ}-12^{\circ}$ left-sided downward tilt. The peritoneal cavity is 
inspected for metastatic disease to confirm the feasibility of resection, and the omentum is then displaced cephalad to allow retraction of the small bowel into the left upper quadrant. The robotic cart is positioned on the same side as the pathology. The $0^{\circ}$ robotic camera is inserted into the supraumbilical port, a monopolar scissor is inserted in the left upper quadrant port, a bipolar fenestrated grasper is inserted in the infraumbilical port, and a Cadiere is inserted in the right lower quadrant port.

We routinely perform medial-to-lateral dissection, beginning along the superior mesenteric vein to locate the origin of the ileocolic pedicles, consistent with the principles of complete mesocolic excision. In the right lower quadrant, arm 4 provides lateral and anterior traction to the cecum and terminal ileum to generate tension for lifting the ileocolic vessels. The retroperitoneum is incised along the path of the superior mesenteric vein, and all nodal tissue above the vessel is cleared with the specimen. The ileocolic pedicle is identified, the retroperitoneal space immediately below the vessels is entered, and a retro-mesenteric dissection is developed. The duodenum and the head of the pancreas are displaced posteriorly, and the ileocolic artery and vein are ligated and divided using the vessel sealer at their origins (Fig. 2). The dissection continues cephalad along the superior mesenteric artery and vein to expose the middle colic vessels and gastrocolic trunk using an infracolic approach. The improved visualization, articulating instruments, and retraction of the robotic platform are particularly useful for ensuring dissection of all nodal disease at the base of the middle colic pedicle (for proximal and mid transverse colon lesions) or right branch of the middle colic pedicle (for cecal and ascending colon lesions; Fig. 3).

Dissection then continues both laterally and cephalad over Gerota's fascia toward the lateral congenital parietal attachments and underside of the hepatic flexure. The hepatic flexure is next addressed; the assistant retracts the transverse colon caudally using the right lower quadrant and subumbilical arms and the surgeon dissects using the left upper quadrant arm (Fig. 4). The gastrocolic ligament is separated from the transverse colon, beginning at the falciform ligament, entering into the lesser sac, continuing proximally toward the hepatic flexure, and separating the omentum off the superior border of the proximal and mid transverse colon.

For extracorporeal anastomosis, the mid and distal transverse colon should be mobilized to ensure sufficient laxity to allow exteriorization of the bowel. The mesentery of the terminal ileum is released from the retroperitoneum, and the congenital peritoneal attachments are incised while holding the inferior pole of the cecum and appendix, continuing dissection up the right paracolic gutter to complete the medialization of the right colon. The terminal ileal mesentery should be fully mobilized to the duodenum to ensure a tension-free anastomosis, often facilitated by increasing Trendelenburg positioning.

For extracorporeal anastomosis, the robot is undocked after a grasper has been placed on the ileocecal junction. The umbilical incision is lengthened to accommodate a wound protector, and the colon is then delivered through the wound. The resection and anastomosis are completed in the usual fashion, and the colon is then returned into the abdomen. ${ }^{57,73}$ 
Intracorporeal anastomosis is especially preferred for patients with elevated body-mass index (BMI), shortened transverse colon mesentery, and proximal transverse colon lesions, since transverse colon mobilization is not required and the specimen can be removed via a Pfannenstiel incision. The stapler is generally introduced via a midleft lateral port, and an isoperistaltic anastomosis is created with suture closure of the common enterotomy and/or colotomy (Fig. 5).

Evidence supporting the use of robotic proctectomy-As with colectomy, robotics could reduce reliance on open approaches for rectal cancer surgery. Adoption of laparoscopy for proctectomy has been even slower, likely because of its technical demands. ${ }^{13,74-76} \mathrm{TME}$ requires a high degree of precision, which can be challenging using long, nonarticulating instruments available in laparoscopy. Laparoscopic TME is especially difficult in males, patients with very low tumors, and obese patients, as surgical access and exposure are markedly restricted. ${ }^{77}$ These factors explain the procedure's high learning curve; as many as 50-150 cases are required to achieve consistent results. ${ }^{36,78}$ Thus, while laparoscopic TME for rectal cancer is oncologically equivalent to and offers short-term advantages over the open approach, it has not gained widespread acceptance among rectal cancer surgeons. 13,16,41,44,79-85 The superior precision and dexterity of the robotic system gives it a clear advantage over conventional laparoscopy in rectal cancer surgery. ${ }^{16,86-88}$ The use of 4 operating arms provides improved retraction, making reliance on a highly skilled assistant less critical, and the robotic instruments' precise articulation enables the surgeon to follow the contours of the rectum with greater ease. ${ }^{1,16}$ In addition, the high-definition 3dimensional camera provides ideal visualization of the pelvic structures.

Although the quality of evidence for robotic TME remains limited, the data accumulated thus far suggests that robotic TME is equivalent to laparoscopic TME in terms of outcomes (Table 1). In the ROLARR trial, which included 466 patients randomized to either procedure, circumferential resection margin (CRM) positivity rates were similar (5.1\% and $6.2 \%$ for the robotic and laparoscopic groups, respectively). These rates were lower than those in previous trials comparing open and laparoscopic proctectomy (COLOR II, CLASICC, ALaCaRT, and ACOSOG Z6051). The odds of achieving the highest-standard plane of surgery (mesorectal plane) did not differ between groups. Large retrospective series reported similarly low rates of CRM positivity for robotic and laparoscopic TME, ranging from $0 \%$ to $8 \%$ and from $1 \%$ to $12 \%$, respectively. ${ }^{89-94}$ No series has found a statistically lower rate of CRM positivity for robotic surgery, consistent with pooled results from metaanalyses, ${ }^{95-101}$ as well as analyses of the National Cancer Database (NCDB) of the American College of Surgeons. ${ }^{36,102-104}$ Robotic surgery has been associated with higher rates of complete or nearly complete TME, though only in a single small study. ${ }^{105}$

Survival and recurrence rates following robotic proctectomy appear similar or slightly better than those after laparoscopic or open proctectomy. The NCDB analyses found comparable overall survival at 3 years, ${ }^{102-104}$ and 2 large case series from Korea found comparable rates of overall survival, disease-free survival, and local recurrence at 3 and 5 years for the 2 techniques. ${ }^{89,92}$ A propensity score-matched study with a median follow-up of 40.3 months determined the robotic approach to be a significant prognostic factor for overall $(P=0.004$; hazard ratio, 0.33$)$ and cancer-specific survival $(P=0.016$, hazard ratio, 0.37$) .{ }^{106} \mathrm{In}$ a 
comparison with open TME, the local recurrence rate was significantly lower with robotic TME at 5 years $(3.4 \%$ and $16.1 \%$, respectively; $P=0.024)$, although margin positivity and cancer-specific outcomes were comparable. ${ }^{107}$

Complications following robotic surgery for rectal cancer also appear similar to those of laparoscopic procedures. Published meta-analyses and examination of NCDB data all found that length of stay and the rates of complications, anastomotic leak, and reoperation are comparable for robotic and laparoscopic surgery for rectal cancer. ${ }^{95,96,98,100-103}$ ROLARR reported no significant difference in 30-day complication rates $33.1 \%$ in the robotic arm, $31.7 \%$ in the laparoscopic arm), including the anastomotic leak rate (12.2\% robotic, $9.9 \%$ laparoscopic). Only the RLOAPR trial found a significantly lower postoperative complication rate $(10.4 \%)$ for robotic than for laparoscopic TME $(18.8 \% ; P=0.027)$. Among patients without complications, robotic TME was also associated with faster recovery of bowel function and shorter median length of stay (5 days in the robotic group vs 7 days in the laparoscopic and open groups; $P<0.001$ ).

Rates of conversion to open surgery are consistently lower for robotic (1.1\%-3\%) compared with laparoscopic $(6 \%-7.5 \%)$ surgery, suggesting that robotic platforms may enhance surgeons' ability to complete more challenging cases. ${ }^{95,96,98,100-103}$ The ROLARR trial also found a lower conversion rate with robotic surgery ( $8.1 \%$ vs $12.2 \%$ in the laparoscopic arm), though the difference did not reach significance. Surgeons who had completed approximately 28 robotic procedures had the same odds of conversion as laparoscopic surgeons with triple the case volume experience (91 cases), suggesting that fewer robotic cases are needed to achieve reliable results than are required to reach expert level in laparoscopy. Conversion rates were even lower in RLOAPR: 0\% for robotic and $2.4 \%$ for laparoscopic surgery $(P=0.123)$, possibly related to surgeon experience.

Only a few studies have evaluated autonomic nerve and urogenital outcomes after robotic TME. ${ }^{90,92,108-110}$ A propensity case-matched retrospective study of 556 rectal cancer patients with long-term follow-up averaging 51.8 months found that robotic TME was associated with significantly lower incidence of late voiding dysfunction (as measured by urodynamics or the international prostate symptom score) compared to laparoscopic TME $(0.7 \%$ vs $4.3 \% ; P=0.01) .{ }^{92}$ Similarly, TME has been reported to be associated with earlier recovery of voiding and sexual function compared to laparoscopic TME. ${ }^{108}$ Preservation of sexual function also appears better with robotic TME, at $100 \%$ of sexually active male patients 1 year after surgery compared to $43 \%$ for laparoscopic TME $(P=0.045) \cdot{ }^{90}$ In the ROLARR trial, the 2 treatment arms did not differ significantly in bladder or sexual function 6 months after surgery. ${ }^{26}$

Although numerous publications suggest that oncologic outcomes after robotic rectal resections are comparable to those after open and laparoscopic procedures, final data from large-scale randomized controlled trials evaluating robotic TME are not yet available. ${ }^{16,111}$ Because of the technical challenges of rectal dissection, most of these procedures are still performed using an open or hybrid laparoscopic and/or open approach in the United States. 12,111 Robotic platforms may facilitate safe completion of TME, as suggested by the lower rate of conversion to open surgery. With proper patient selection and sufficient practice, 
robotic TME offers an effective treatment modality in the hands of an experienced rectal surgeon, although these advantages come at a higher financial cost. ${ }^{16,62,74,97,98,105,112-118}$ If current trends continue, the costs associated with robotic surgery will likely decrease, owing to economies of scale, improved instrument design, and increased proficiency of the operating teams. ${ }^{119}$

Robotic rectal TME technique (low anterior resection)-The primary goal of surgical intervention for rectal cancer is obtaining oncologic cure while preserving function. Removing all local tumor cells requires high ligation of the vascular supply at its origin, as they spread cranially along the superior rectal vessels. Because tumor rarely spreads distally beyond $1 \mathrm{~cm}$, distal margins of that length are adequate for control of disease and sphincter preservation. ${ }^{120-122}$ For a double-stapled anastomosis after TME, the surgical distance between the lower edge of the tumor and the anorectal ring should ideally be at least 1 to 2 $\mathrm{cm}$. Adjacent nerves must be spared to avoid genitourinary dysfunction; these include the sympathetic nerves originating from L1 and L3 that extend as the hypogastric nerves along the sacrum and the parasympathetic nervi erigentes, which originate from S2 to S4 and join the hypogastric nerves anterior and lateral to the rectum.

Rectal resections can be divided into 2 major stages: (1) an abdominal stage, which involves mobilization of the left colon and splenic flexure and division of both the inferior mesenteric artery (IMA) and the inferior mesenteric vein (IMV), and (2) a pelvic stage, during which rectal dissection and TME are performed.

Dissection during the abdominal stage can be accomplished in a number of ways, including medial-to-lateral, lateral-to-medial, and IMV first. A medial-to-lateral approach allows easy visualization and control of the mesenteric vasculature early in the procedure, immediate delineation of the plane between the mesentery and the retroperitoneum, preservation of the autonomic nerves, early identification of the left ureter and other retroperitoneal structures, and quick access to the splenic flexure.

Certain steps in the robotic TME procedure require particular attention to avoid complications. High ligation of the IMA and division of the IMV near the ligament of Treitz facilitate colonic mobilization and creation of a tension free anastomosis. Vascular division should be performed after the left ureter is identified, as this structure travels lateral to and in very close proximity to the IMA. Avoiding damage to autonomic nerves requires special attention in the following anatomic areas: (1) the superior hypogastric plexus during dissection of the IMA, (2) the hypogastric nerves at the sacral promontory during entry into the retrorectal space, (3) the pelvic plexus during lateral mobilization of the rectum, and (4) below the peritoneal reflection during anterior dissection of the rectum.

The patient is placed in the modified lithotomy position, with the buttocks slightly over the end of the table. The thighs are abducted and aligned with the contralateral shoulder. The hips, particularly on the left side, should be fully extended, and the knees should be flexed at $45^{\circ}$ so that the legs are not in the way when the robot is docked at the left hip. Both legs should be gently rotated internally to avoid lateral pressure on the peroneal nerve. The patient's arms are placed alongside the body to lessen the possibility of shoulder injury and 
to provide sufficient space for the surgeon and assistant as well as the robotic platform.

Pressure points and bony prominences are padded, and the body is secured to the operating table. Before proceeding, the patient's secure positioning is confirmed by testing the table in the Trendelenburg position and in a left-sided tilt.

We prefer to obtain pneumoperitoneum using a Veress needle below the left costal margin in the midclavicular line, as it is safe and technically easy, even in morbidly obese patients. The camera port is placed just above the umbilicus, and $8 \mathrm{~mm}$ ports are placed in the right upper quadrant, right lower quadrant, and left mid abdomen. A $12 \mathrm{~mm}$ trocar is placed roughly halfway between the umbilicus and right anterior superior iliac spine, which corresponds to the midclavicular line, and often can be used as an aperture for a diverting loop ileostomy. An accessory port is also placed lateral in the right mid abdomen (Fig. 6).

For pedicle ligation and splenic flexure mobilization, ports $1 \mathrm{a}, 2$, and 3 are utilized, with the patient in a slight Trendelenburg position with a $12^{\circ}$ to $15^{\circ}$ tilt. The small bowel is swept laterally, and the omentum is placed over the liver, exposing the IMA and IMV. A bipolar fenestrated grasper is used in the right upper quadrant port, the monopolar scissor is used in the right lower abdominal port, and the Cadiere grasper is used in the lowest right quadrant port. With the Cadiere holding tension on the sigmoid mesentery, the peritoneum at the sacral promontory is incised, the autonomic nerve is swept into the retroperitoneum, and the IMA is identified. In a medial-to-lateral dissection, the sigmoid mesentery is dissected off the retroperitoneum in an avascular plane with identification of the gonadal vessels laterally and the ureter medially. Next, the IMV is isolated and divided at the level of the ligament of Treitz and just inferior to the pancreas (Fig. 7). The left colon is mobilized off the retroperitoneum medially to laterally in an avascular plane.

In very obese patients, starting the dissection at the level of the IMV has the advantage of entering the avascular plane between the retroperitoneum and the mesocolon early. The IMV is a reliable landmark whose position varies little among individuals, so starting the dissection here is very reproducible.

The splenic flexure is then mobilized by further elevating the left colon mesentery off the retroperitoneum and then off the pancreas with entry into the lesser sac (Fig. 8). Next, the omentum is dissected off the distal transverse colon, and the left colon lateral wall attachments are divided along with the remaining retroperitoneal attachments of splenic flexure colon.

After the IMV is divided and the left colon is mobilized, the IMA should take on the characteristic T-shaped structure (Fig. 9). The position of the ureter and gonadal vessels in the retroperitoneal plane is reconfirmed and the IMA is ligated with the Vessel Sealer. The medial-to-lateral dissection continues toward the abdominal wall using blunt dissection to gain entry into the previously developed avascular plane beneath the IMV and advance toward the sacral promontory. As the gonadal vessels and the ureter are encountered, they should be swept posteriorly toward the retroperitoneum. Visualization of the psoas muscle usually indicates that the dissection is in an incorrect and deep plane. 
Following division of both the IMA and the IMV, the lateral attachments of the sigmoid and descending colon are divided along the line of Toldt. This dissection, starting at the left lower quadrant, is facilitated by retracting the colon medially and anteriorly. Division of the line of Toldt reveals the medial dissection plane; as dissection progresses toward the left upper quadrant, any omental attachments to the colon should be divided, leaving attachments between the omentum and the abdominal wall in place, unless visualization is impaired.

The entire left colon should now be medialized and mobilized so that attention can be directed at the proposed site of mesenteric ligation and colon transection for future anastomoses. The mesentery of the descending colon is then divided from the stump of the IMA toward the colon to the point of the future division of the bowel, usually at the junction of the descending and sigmoid colon. The mesentery is divided with a Vessel Sealer with care to divide the marginal artery to avoid tearing the vessels during extraction.

After colonic mobilization, pelvic dissection can begin. The robotic arms are detached from the trocars, and the patient is leveled and placed in a significant Trendelenburg (20 $0^{\circ}$ to $25^{\circ}$ ) position to keep the small intestines out of the pelvis. The robotic system should be redocked at the patient's left hip, permitting access to the anus and perineum (Fig 6). As the assistant elevates the rectosigmoid junction (or grasps the divided mesenteric pedicle of the superior rectal artery), dissection begins posteriorly at the sacral promontory, entering the avascular plane between the visceral and parietal layers of the endopelvic fascia (Fig. 10). ${ }^{75}$

At the beginning of dissection, the hypogastric nerves should be gently pushed away from the plane of dissection. As dissection continues distally, the surgeon must keep in mind that the rectum curves upward and anteriorly as the anorectal junction is approached. Just above the levator ani muscles, the endopelvic fascia fuses with the mesorectal fascia. To avoid bleeding and injury to the fascia, the dissection uses monopolar cautery, and the mesorectum is manipulated using a gauze tie rather than robotic graspers. The TME proceeds along the areolar plane down to the rectococcygeal ligament.

Anteriorly, the peritoneal reflection is incised, and the dissection is continued along the rectovaginal septum in women or over the rectovesical or rectal prostatic fascia (Denonvilliers fascia) in men (Fig. 11). Arm 3 is used to retract the bladder and other anterior structures as dissection proceeds distally. The articulation of the robotic scissor tips enables the surgeon to perform the dissection using ideal approach angles: as distally as possible in the posterior plane, which facilitates identification of the lateral stalks and dissection in the anterolateral areas. In most cases, the surgeon alternates between the posterior, lateral, and anterior planes to achieve complete circumferential dissection.

Laterally, dissection proceeds along the sidewalls medial to both ureters, contouring along the curving mesorectal plane. Injury to the autonomic plexus and generation of excess medial traction on the sidewall, which jeopardize transection of the nervi erigentes, should be avoided. As the lateral stalks are divided, care should be taken to preserve the hypogastric plexus and the pelvic sidewall, lateral to the seminal vesicles in men and the cardinal 
ligaments in women. The lateral stalks are controlled with bipolar cautery or monopolar cautery using the scissors and divided.

Anteriorly, the peritoneum between the rectum and seminal vesicles or upper vagina is dissected under direct vision by simultaneous retraction of the anterior structures toward the pubis, and the rectum toward the sacrum. During this dissection, the planes are less distinct, and the fat on the anterior mesorectum can be thin, so the anterior pelvic structures are elevated off the anterior rectal wall. The dissection continues through Denonvilliers fascia, which is separated from the anterior structures and kept with the specimen. The distal point of this dissection matches that of bowel transection, which depends on the level of the tumor. Middle and distal rectal tumors require removal of the entire mesorectum, while an upper rectal tumor requires transection of the rectum and mesorectum $5 \mathrm{~cm}$ below the level of the tumor. ${ }^{120}$

Dissection continues down to the pelvic floor, separating the fatty mesorectum from the levator muscle. The rectum is lifted off the muscle and cleared circumferentially for transection. This mobilization of the rectum increases the distance of the tumor from the dentate line, allowing an adequate distal margin and preservation of the sphincters. Continuing dissection further down allows the surgeon to access the intersphincteric plane when performing ultralow anterior resection with intersphincteric dissection. In preparation for rectal division, the rectum is examined digitally and by flexible endoscopy to ascertain the level of the tumor. Using the $12 \mathrm{~mm}$ trocar port, a $45 \mathrm{~mm}$ robotic stapler is used to divide the rectum, after which the robotic cart can be undocked. We routinely extract the specimen by enlarging the right lower quadrant port site (later used as the site for diverting ileostomy) or umbilical port site to $3-5 \mathrm{~cm}$ to permit a wound protector. Alternatively, a Pfannenstiel incision can be utilized. The proximal bowel is divided, and an anvil is secured to the proximal colon with a purse-string suture. The descending colon conduit is returned to the peritoneal cavity, the wound protector is occluded, and pneumoperitoneum is reestablished. The circular stapler is introduced through the anus and an end-to-end anastomosis is constructed under robotic vision (Fig. 12).

Summary-Robotics is a natural extension of laparoscopy and has broadened the application of minimally invasive surgery to colorectal oncology. Controversy remains as robotic systems are expensive, and investigations will continue to determine how best to utilize this new technology.

\section{Robotic pancreatic surgery}

\section{Rajesh Ramanathan, Melissa E. Hogg, Amer H. Zureikat, and Herbert J. Zeh}

Pancreatic operations are technically challenging endeavors due to the central location of the pancreas within the retroperitoneum, intimate association with key vascular structures, soft texture, and intolerance of technical errors. Robotic assistance in pancreas surgery offers several advantages. These include improved 3-dimensional retroperitoneal visualization at the level of the pancreatic neck and uncinate process, optical magnification around key vascular structures, technical stabilization of tremors, and near- $540^{\circ}$ articulation of the robotic instruments. ${ }^{123}$ The technical advantages offered by robotics in pancreas surgery are 
reflected in the decreased conversion to open rates as compared to conventional laparoscopy. 124

Here, we review the data and technique for several robotic pancreas operations and discuss standardized training and the learning curve in robotic pancreas surgery.

Proficiency and learning curve-As robotic surgery becomes increasingly common across the surgical disciplines, it remains important to continually assess learning curves, proficiency, outcomes, and comparative effectiveness. Robotic pancreatic surgery has a unique learning curve associated with platform-familiarity, visual cues as a substitute for the absence of tactile feedback, and navigation within a tight working space. Additionally, the paradigmatic challenges of training and certifying surgeons on the robotic platform must be confronted. In this section, we discuss standardized training curricula, learning curves, and intraoperative evaluation in robotic pancreas surgery.

The need for a global, standardized training curriculum has been described. ${ }^{125}$ The Fundamentals of Robotic Surgery is a proposed, international, consensus curriculum emphasizing 3 essential components: cognitive skills, psychomotor skills, and team training and communication skills. ${ }^{126}$ Results from implementation of that curriculum and outcomes are pending.

The University of Pittsburgh Medical Center (UPMC) has developed a robust, proficiencybased, training program for robotics with similar objectives, placing an emphasis on pancreas surgery. The curriculum includes mastery-based virtual reality simulation, an inanimate biotissue curriculum, video library training, intraoperative evaluation, and skill maintenance that have previously been described in detail. ${ }^{125,127}$ Results from this program show that surgical fellows completing the curriculum subjectively find value and objectively demonstrate improvements in technical errors, technical skill, and speed. ${ }^{128}$

UPMC is fortunate to have 1 of the world's largest experiences in robotic pancreatectomy, enabling granular quantification of the learning curve and outcomes for robotic pancreaticoduodenectomy (RPD) and robotic distal pancreatectomy (RDP). ${ }^{129-131}$ For RPD, analysis of 200 consecutive operations at UPMC demonstrated that after 20 cases, statistically significant improvements were noted in blood loss and conversion to open surgery, while after 40 cases, rates of postoperative pancreatic fistulae (POPF) were cut nearly in half $(27.5 \%-14.4 \%) .{ }^{129}$ Notably, reduction in operative time had the longest learning curve, up to 80 cases to decrease from 581 to 417 minutes. Napoli and colleagues have also published their learning curve for RPD, reporting 33 cases to decrease operative time from 563 to 484 minutes, and 40 cases to decrease readmissions from $20 \%$ to $3.3 \%$. ${ }^{132}$ Perhaps commensurate with the complexity of the operation, the learning curve to reduce operative time for RDP was 40 cases in the UPMC series, and 10 cases in the series by Napoli and colleagues. ${ }^{130,133}$ These learning curves emphasize the need for standardized mastery-based curricula for trainees and new entrants to robotic pancreas surgery. ${ }^{134}$

Another important component is intraoperative evaluation and continued maintenance. Our data show that robotic technical proficiency is an independent predictor of outcomes, 
whereby blinded grading of the duct-to-mucosa pancreaticojejunostomy (PJ) during RPD correlates with the incidence of POPF. ${ }^{135}$ Similar ongoing initiatives aim to find new ways to monitor intraoperative performance and provide technical feedback to surgeons.

RPD—Pancreaticoduodenectomy (PD) is among the most challenging gastrointestinal operations, with well-documented surgeon and institutional learning curves for open operations. ${ }^{136,137}$ RPD has thus served as a barometer for assessing technical proficiency of individual surgeons, while simultaneously evaluating the value of robotics in major gastrointestinal surgery. The evidence to date suggests that RPD performed by high-volume surgeons past their learning curve achieves comparable oncologic outcomes, complication rates, and possibly faster recovery. ${ }^{138,139}$

There is global interest in RPD, with a range of published outcomes. From China, Chen and colleagues analyzed outcomes in a series of 60 RPD matched with 120 open PD. They report longer operative time for RPD (410 minutes vs 323 minutes), but significantly less intraoperative blood loss, better recovery of postoperative protein status, faster return to ambulatory activity and bowel function, and shorter hospital stay (20 days vs 25 days), with no difference in complication rate. ${ }^{140}$ Oncologically, they had similar R0 resection rates, lymph node yield, and disease-free survival for adenocarcinoma. Higher operative costs were partially offset by lower postoperative costs. Another report from China had a similar decrease in hospital stay and blood loss when compared to an open cohort. ${ }^{141}$

North American series have uniformly reported shorter hospital stays. Buchs and colleagues analyzed 83 consecutive patients undergoing open and robotic PD, reporting shorter operative time, less blood loss, increase lymph node yield, and otherwise no difference in oncologic outcomes with RPD. ${ }^{142}$ A smaller series from Cleveland Clinic reported outcomes of 30 matched robotic and open PD, reporting longer operative times and shorter hospital stays with RPD. ${ }^{143}$ In our experience at UPMC, we have reported outcomes before and after our learning curve. ${ }^{129}$ Past the learning curve, our outcomes include 417 minutes mean operative time, mean blood loss of $250 \mathrm{~mL}$, conversion rate of $3.3 \%$, median length of stay of 9 days, $6.9 \%$ clinically significant grade B or C POPF, and 90-day mortality of $3.3 \%$. In a propensity-matched analysis, we found no difference in POPF or other postoperative outcomes in RPD as compared to open PD. ${ }^{144}$ Notably, we found that RPD may offer unique benefits over open PD in morbidly obese individuals, whereby RPD serves to decrease operative time, blood loss, and wound infection as compared to open PD. ${ }^{145}$

\section{RPD technique}

Patient selection: The indications for RPD do not vary significantly from the selection criteria for open PD. Specifically, we do not consider BMI or medical comorbidities as exclusionary criteria. ${ }^{146}$ The robotic approach has been feasible, safe, and oncologically equivalent among patients requiring vein resections, and among those with anomalous arterial anatomy. ${ }^{147}$

In the setting of pancreatic ductal adenocarcinoma, we routinely refer patients with borderline resectable or local advanced disease for neoadjuvant chemotherapy, with or without stereotactic body radiation therapy. ${ }^{148}$ Radiographic and biochemical response is 
followed while on neoadjuvant therapy, and RPD is offered for select patients with locally advanced disease 27-31,149-153

Instruments and positioning: The platform currently used at UPMC for RPD is the da Vinci Si (Intuitive Surgical, Sunnyvale, California). RPD is a 4-handed operation, requiring a skilled bedside laparoscopist in addition to the console surgeon. Our practice utilizes the monopolar robotic hook for dissection, the robotic bipolar for hemostasis, laparoscopic LigaSure Blunt Tip (Covidien, Medtronic, Dublin, Ireland) for dividing vascularized tissue, and an articulating laparoscopic linear stapler (Covidien, Medtronic, Dublin, Ireland) for division of vascular and visceral structures.

The patient is positioned supine in a split-leg configuration with the right arm tucked to accommodate the arms of the robot. Following intubation, the bed is rotated approximately $45^{\circ}$ to accommodate the robot which enters in a craniocaudal axis over the patient's head (the bedside laparoscopist ultimately occupies the space between the legs). A wide surgical preparation is used to accommodate the lateral robotic arm and the liver retractor.

Peritoneal access and port placement: Typical port placements are shown in Fig. 13. Our practice is to gain peritoneal access using a $5 \mathrm{~mm}$ optical separator in the left subcostal region. A $10 \mathrm{~mm}$ camera port is placed approximately $2-3 \mathrm{~cm}$ to the right of the umbilicus. The $8 \mathrm{~mm}$ robotic ports are placed $2-3 \mathrm{~cm}$ cephalad to the camera port with 2 right-sided and 1 left-side port lateral to the midclavicular line. The laparoscopic assistant ports are placed infraumbilical about 5-6 cm caudal to the camera port with 1 right sided $5 \mathrm{~mm}$ port, and a second $12 \mathrm{~mm}$ port that also functions as the specimen extraction port. A left lateral $5 \mathrm{~mm}$ port serves as the access for the liver retractor in the anterior axillary line.

Colonic mobilization and extended duodenal Kocher: The patient is placed in steep reverse Trendelenburg position and the robot is docked. Once the liver retractor has been positioned, the first part of the operation involves mobilization of the right colon, and dissection of the third and fourth portions of the duodenum to deliver these structures to the right upper quadrant.

The gastrocolic ligament is divided to enter the lesser sac lateral to medial, identifying the right gastroepiploic pedicle. The hepatic flexure of the right colon is mobilized inferiorly to the terminal ileum to allow for a complete right medial visceral rotation. This allows visualization of the superior mesenteric vein (SMV) at the root of the mesentery. A complete mobilization of the duodenum and head of pancreas is performed with a generous Kocher maneuver. The Kocher maneuver is extended to divide the ligament of Treitz and deliver the proximal jejunum to the right upper quadrant. This facilitates division of the jejunum approximately $10 \mathrm{~cm}$ distal to the ligament of Treitz with the linear stapler.

Lesser sac entry and division of the stomach and/or duodenum: Dissection of the lesser sac is completed to free the posterior stomach from the anterior pancreas. The distal stomach is cleared of mesentery along the lesser and greater curvatures and divided with a linear stapler. This provides improved exposure of the superior aspect of the pancreas. In pylorus- 
preserving situations, the duodenum is divided with a linear stapler distal to the pylorus with a $2 \mathrm{~cm}$ cuff.

Dissection of the porta hepatis: The Pars flaccida is entered and the loose areolar tissue overlying the porta hepatis is dissected with hook cautery to identify the common hepatic artery (CHA) and the Station VIIIa CHA lymph node (Fig. 14). The VIIIa lymph node is removed for permanent pathology. The right gastric artery is dissected, and divided with a linear vascular stapler or hemoclips near its origin. The gastroduodenal artery (GDA) is identified and encircled with a vessel loop. A test-clamp of the GDA is used to confirm pulsatile flow in the proper hepatic artery, using laparoscopic ultrasound as an adjunct if required. The GDA is then divided with a vascular stapler.

The common bile duct (CBD) is identified and the lateral CBD nodes are cleared. The CBD is dissected medially to expose the medial wall of the portal vein (PV). The CBD is divided distally with either a linear stapler, or with cautery scissors and proximal bulldog placement. The anterior surface of the $\mathrm{PV}$ is dissected proximally to the superior border of the pancreatic neck.

SMV dissection and pancreatic division: The right gastroepiploic vein is traced to its origin to identify the SMV and middle colic vein. The inferior border of the pancreas is carefully dissected to expose the plane between the posterior pancreas neck and the SMV. The retropancreatic avascular plane is dissected in a cephalad dissection to meet with the superior plane anterior to the portal vein at the superior border of the pancreatic neck (Fig. 14). The robotic platform provides the distinct advantage of improved visualization of the entire retropancreatic tunnel. A moistened umbilical tape is passed through the tunnel to aid in the division of the pancreatic neck parenchyma. The pancreas is divided with the hook cautery with use of the bipolar cautery for advanced hemostasis. The pancreatic duct is cut sharply with scissors, and a duct margin is submitted for frozen section analysis in cases of malignancy.

Uncinate dissection: Following division of the neck, the head of the pancreas is mobilized from the lateral border of porto-splenic confluence in a cephalad direction (Fig. 14) Small perforating branches are dividing using the LigaSure device and bipolar cautery for additional hemostasis. The superior pancreaticoduodenal vein is divided with either a vascular stapler clips or LigaSure depending on its size. The posterior SMA plane of Leriche is similarly divided using cautery and the other energy devices to free the specimen (Fig. 14).

Cholecystectomy and specimen extraction: The gallbladder is freed in standard fashion after delineation of the critical view of safety. The gallbladder is removed with use of an Endo Catch bag.

The $12 \mathrm{~mm}$ assistant port skin and fascia are extended laterally and a gel port appliance is used to facilitate extraction of the specimen with an Endo Catch bag. Following removal of the specimen, the $12 \mathrm{~mm}$ port is placed through the gel hand port and insufflation is achieved again for the reconstruction phase of the operation. 
Reconstruction: The pancreaticobiliary limb is brought behind the mesenteric vessels to lay in the right upper quadrant. A modified Blumgart 2-layer, end-to-side, duct-to-mucosa pancreaticojejunostomy $(\mathrm{PJ})$ anastomosis is fashioned. Transpancreatic, horizontal mattress sutures using 2-0 silk sutures are affixed to the seromuscular layer of the jejunum to form the outer layer of the 2-layer anastomosis. A small enterotomy is made with the cautery scissors and an interrupted duct to mucosa anastomosis is performed with 5-0 Maxon suture (Covidien, Medtronic, Dublin, Ireland) (Fig. 15). It is our practice to use a pancreatic duct stent (4-7 French, $7 \mathrm{~cm}$ Zimmon pancreatic stent, Cook Medical, Bloomington, Indiana).

Approximately $10-15 \mathrm{~cm}$ distal to the PJ, the hepaticojejunostomy (HJ) is fashioned. For ducts greater than $5 \mathrm{~cm}$, a single-layer, running anastomosis with 2 4-0 V-Loc barbed sutures (Covidien, Medtronic, Dublin, Ireland) is performed (Fig. 15). For smaller ducts, an interrupted, single-layer anastomosis with 5-0 PDS suture is performed with selective stenting.

Approximately $40-50 \mathrm{~cm}$ distal to the $\mathrm{HJ}$, the jejunum is brought up to the stomach in an antecolic configuration to the right of the middle colic vein. A robotically hand-sewn, 2layer, Hoffmeister end-to-side gastrojejunostomy is performed. An approximately $6 \mathrm{~cm}$ gastrojejunal anastomosis is fashioned using an inner layer of 2 running 3-0 V-Loc barbed sutures, and 3-0 silk Lembert sutures for the outer layer.

Drains and closure: After ensuring hemostasis, a single 19 French round surgical drain is placed across the pancreatic and biliary anastomoses. The extraction port and $10 \mathrm{~mm}$ camera port fascia are closed and the skin sites are all closed with subcuticular monofilament suture.

Patients are taken to recovery and admitted to a monitored unit and do not require routine admission to the intensive care unit. ${ }^{154}$

RDP-Minimally invasive laparoscopic DP (LDP) has been extensively studied in benign and malignant disease, and is considered the preferred operative approach over open DP. $11,155,156$ With regards to RDP, many smaller series with experiences of less than 50 cases have reported equivalence between LDP and RDP. ${ }^{157-159}$ The learning curve for RDP, especially in operative time, has been reported by Napoli at 10 cases, and by our group at 40 cases. ${ }^{130,133}$ It is important to note that this is the learning curve following extensive institutional experience with RPD at both centers. In fact, larger series, with surgeons presumably past their learning curve, have shown comparative advantages with RDP over LDP. $^{160-162}$

In a matched comparison of $69 \mathrm{RDP}$ and $50 \mathrm{LDP}$, Chen and colleagues demonstrated greater rates of splenic preservation, shorter operative times, less blood loss, and shorter hospital stays. ${ }^{160}$ At UPMC, we have reported lower rates of conversion to open surgery at $2 \%$, improved rates of negative margin resection, and higher lymph node yield. ${ }^{162}$ The lower conversion rate and increased rates of splenic preservation are likely functions of the $540^{\circ}$ articulation of the robotic instruments, which enables more nuanced splenic artery and vein dissection. 


\section{RDP technique}

Patient selection: There are no absolute contraindications to a robotic approach for DP.

Relative contraindications include locally advanced malignancies, multivisceral involvement, vascular invasion, and prior abdominal operations. Celiac involvement is not an absolute contraindication, and will be discussed in subsequent sections.

Positioning and port placements: Similar to RPD, the da Vinci Si platform is used with the monopolar robotic hook, the robotic bipolar, laparoscopic LigaSure, and the articulating laparoscopic linear stapler.

The patient is placed supine in a split leg position, and intraperitoneal access and insufflation is gained using an optical separator in the left subcostal, midclavicular line. A $12 \mathrm{~mm}$ trocar is placed supraumbilical to the right of the umbilicus. Robotic $8 \mathrm{~mm}$ trocars are placed in the left paraxiphoid, right subcostal, and left supraumbilical areas (see Fig. 13b).

Lesser sac access and mobilization of the splenic flexure: The robot is docked, and the gastrocolic ligament is opened widely to enter the lesser sac. The splenic flexure is then mobilized, and reflected caudally to fully expose the anterior surface of the pancreas. The transverse mesocolon is dissected away to exposure the inferior border of the pancreas.

Pancreatic division: Laparoscopic ultrasound is a useful adjunct for masses that are not grossly visible, and to identify the proximal transection plane. The superior border of the pancreas is dissected at the chosen transection point, and a careful retropancreatic tunnel is created. The robot offers improved ability to visualize and avoid injury to the splenic vessels if splenic preservation is planned. Passage of a moistened umbilical tape allows upward traction and division with a linear stapler (Fig. 16).

For formal distal pancreatectomies, the avascular tunnel above the SMV at the level of the neck is developed under direct visualization similar to RPD. If concurrent splenectomy is planned, the splenic artery can be identified at its takeoff from the celiac trunk and divided with a vascular linear stapler (Fig. 16).

Medial to lateral dissection: The posterior retroperitoneal attachments of the pancreas are dissected in a medial to lateral direction to achieve complete mobilization of the specimen. For malignancies requiring formal lymphadenectomy, a complete lymphadenectomy is similarly performed medial to lateral starting at the lateral border of the superior mesenteric artery (SMA). Removal of the posterior pancreatic fascia en bloc with a complete celiac lymphadenectomy provides excellent lymph node yield, and enables a sound oncologic resection (Fig. 16). For splenectomy, the spleen is mobilized in a lateral to medial fashion.

Specimen extraction: The specimen is removed through an Endo Catch bag at the $12 \mathrm{~mm}$ port site. It is our routine practice to leave a single 19 French round surgical drain in the pancreatic bed. The extraction port and $10 \mathrm{~mm}$ camera port fascia are closed and the skin sites are all closed with subcuticular monofilament suture.

Curr Probl Surg. Author manuscript; available in PMC 2019 August 08. 
RDPCAR-Distal pancreatectomy with celiac axis resection (DPCAR), also known as the modified-Appleby procedure, is indicated for select locally advanced tumors of the pancreatic body that involve the celiac axis, but spare the SMA and GDA. Reports of DPCAR detail the procedure's safety, high rate of R0 resections, and improved survival and quality of life. ${ }^{163-165}$ At UPMC, we retrospectively compared 19 open DPCAR to 11 RDPCAR and reported decreased operative time, reduced blood loss, and no need for conversion with the robotic approach. ${ }^{166}$ There was no difference in $\mathrm{R} 0$ resection and morbidity and mortality.

RDPCAR technique-Patient position, instruments, and port placements are similar to RPD. RDPCAR has been described in detail previously, and is summarized below. ${ }^{166}$

Medial dissection: After entry into the lesser sac, the CHA is dissected at the superior border of the pancreas and traced distally to identify the GDA. The CHA is then testclamped with use of laparoscopic ultrasound to ensure adequate collateral flow through the GDA to the hepatic artery proper. The retropancreatic tunnel is created at the level of the neck, and the pancreas is divided. The CHA is then transected with a linear vascular stapler, taking care to preserve the GDA (Fig. 17).

Typically, the splenic artery is encased by tumor; therefore, we next identify the splenic artery near the splenic hilum to ligate the inflow prior to venous transection to prevent engorgement and back-bleeding. Following division of the CHA, the dissection proceeds deep and posterior to the pancreas. The splenic vein is divided with a linear stapler. Dissection continues to identify the SMA, which is traced to its origin at the aorta. This facilitates identification of the celiac axis and allows for the lymphadenectomy and delineation of the inferior aspect of the celiac axis.

Anterior dissection: The divided CHA is traced to its origin at the celiac trunk. The encountered left gastric pedicle is divided distal to its takeoff, and a complete lymphadenectomy is performed along the superior-medial aspect of the celiac trunk.

Lateral dissection: The splenic flexure is mobilized and reflected caudally. The spleen and pancreatic tail are mobilized from the retroperitoneum in a lateral to medial fashion. The retroperitoneal fascia is carried medially toward the aorta, thereby completing the lateral aspect of the celiac lymphadenectomy.

Celiac trunk transection: The celiac is also approached cranially by dissecting the right crus to the aorta which allows for a well-visualized circumferential dissection. Having achieved circumferential dissection and lymphadenectomy at the root of the celiac trunk, a linear vascular stapler is used to divide the celiac artery (Fig. 17). The specimen is removed through the $12 \mathrm{~mm}$ port and a 19 French round surgical drain is placed in the bed.

$\mathbf{R C P}-$ Central pancreatectomy $(\mathrm{CP})$ is an infrequently performed procedure indicated for benign or indolent lesions of the pancreatic neck that do not require DP. CP may decrease the risks of postpancreatectomy diabetes and exocrine insufficiency. ${ }^{167,168}$ There are few reports of RCP, in large part due to the rarity of the operation itself. 
Initial experiences of RCP consisted of small single-center series. In South Korea, Kang and colleagues reported a series of 5 patients, 4 of whom had a solid pseudopapillary tumor, and a fifth with a neuroendocrine tumor. ${ }^{169}$ One patient had a grade B POPF. Retrospective comparison with open $\mathrm{CP}$ at their institution found longer operative time with RCP, but less intraoperative blood loss. Giulianotti and colleagues also published a series of 3 patients with cystadenomas who underwent RPD with pancreaticogastrostomy (PG) and pancreaticoenterostomy. ${ }^{156}$ The published experience with RCP at UPMC consists of 13 cases. ${ }^{170}$ We reported median blood loss of approximately $200 \mathrm{~mL}$ and POPF in 12 of the 13 cases. Of those fistula, 9 were grade $\mathrm{B}$ requiring drainage with 1 grade $\mathrm{C}$ POPF. Two cases required conversion to open.

The largest institutional report to date is a randomized control trial from China comparing RCP to open CP in 100 patients. RCP was associated with shorter length of stay (15.6 vs 21.7 days), shorter median operative time, less blood loss, and lower rate of POPF (18\% vs $36 \%) .{ }^{171} \mathrm{~A}$ summary of the literature on CP demonstrated that across multiple studies, minimally invasive CP had a lower rate of mean morbidity (37\% vs $43 \%$ ) than open CP. ${ }^{172}$ The overall fistula rate was $36.6 \%$, however the rate of clinically significant grade B and C fistulas was $17 \%$. Development of postoperative diabetes was $1.5 \%$ and there were no reports of exocrine insufficiency.

RCP technique-Our technique for RCP, including supplementary video, has been previously described, and will be summarized below. ${ }^{173}$

Patient positioning, instruments and port placement are similar to RPD. The lesser sac is opened to expose the anterior surface of pancreas. Laparoscopic ultrasound can be used to identify the tumor, if necessary.

The inferior pancreatic border is dissected to identify the SMV as it passes behind the neck of the pancreas. The CHA is dissected along the superior border with sampling of the CHA node. The PV is identified and the retropancreatic tunnel is created with passage of the umbilical tape behind the neck of the pancreas. The proximal transection is performed with a vascular linear stapler or with cautery depending on the gland texture. When not using the stapler, the parenchyma is divided with cautery and oversewn with 2-0 silk horizontal mattress suture, and the duct is ligated with 4-0 polydioxanone suture. The central pancreas is then lifted and dissected medial to lateral away from the splenic vein. The distal margin is identified and the pancreas is divided with cautery. The specimen is removed.

For the reconstruction a PG and PJ (identical to RPD) have both been performed depending on surgeon preference. For the PG, the greater curve of the stomach is mobilized sufficiently to perform PG. The anterior surface of the pancreas remnant is anchored to the posterior stomach and a modified Blumgart 2-layer duct-to-mucosa anastomosis is performed with a pancreatic stent similar to the PJ described in the RPD.

RTP-Total pancreatectomy (TP) can be performed without or with islet autotransplantation (TP-IAT). The indications for TP alone include synchronous tumors along the head and body and/or tail, persistently positive margins after PD or DP, intraductal papillary mucinous 
neoplasm with field defects affecting the entire length of the gland, and refractory chronic pancreatitis. ${ }^{174}$

TP-IAT is a specialized operation for early chronic pancreatitis aimed at relieving the endocrine deficiency by reimplantation of isolated, washed, autogenous islets. Unique technical considerations in TP-IAT include minimization of warm ischemia time and parenchymal injury to maximize islet yield.

Galvani and colleagues published a case series of 6 patients with chronic pancreatitis that underwent RTP-IAT with $630 \mathrm{~mL}$ mean blood loss, no complications or conversions, and evidence of successful islet function. ${ }^{175}$ Our institution has reported a series of 9 RTP and 1 RTP-IAT with similar operative time and blood loss. There was 1 conversion to laparotomy. 176

RTP and RTP-IAT technique-The RTP, including IAT, technique has been previously described with video supplementary material, and will be summarized below. ${ }^{176}$

RTP: Port placements are similar to the setup for RPD. Similar to the RPD, the CHA, GDA, $\mathrm{PV}$, and SMV are exposed. The GDA is ligated, and the stomach and CBD are divided. The retropancreatic tunnel is developed and the pancreatic neck is divided. Dissection is then carried out along the inferior border of the pancreatic body in a medial to lateral direction with ligation of the splenic vessels and the IMV. The splenic flexure and splenic attachments are released, allowing a lateral to medial mobilization of the spleen and pancreatic tail. The pancreatic head and uncinate are released similar to RPD and the specimen is removed. Reconstruction with HJ and gastrojejunostomy occurs similar to RPD.

RTP-IAT: For RTP with IAT, there are certain key differences. The pancreatic neck is not divided to maximize islet yield. The retropancreatic umbilical tape helps to elevate the specimen to facilitate dissection of the vasculature. To minimize warm ischemia, the GDA, IMA, and splenic vessels are not ligated until complete mobilization of the specimen (Fig. 18). At that point, heparin is administered intravenously at $50 \mathrm{IU} / \mathrm{kg}$, and the splenic artery, GDA, and splenic vein are divided in that order. A long stump of splenic vein is left for eventual IAT, whereby a 14-gauge angiocath is robotically advanced into the splenic vein stump (Fig. 18).

RCG-Walled-off necrosis (WON) of the pancreas is a common occurrence after acute necrotizing pancreatitis. Endoscopic treatments are considered first-line in many cases. However, although endoscopic cyst gastrostomy is associated with great success in simple pseudocysts, they are complicated by need for repeat interventions in WON due to the viscosity and particulate nature of necrotic pancreata. ${ }^{177}$ In such situations, surgical debridement may be of benefit.

Open necrosectomy is a morbid procedure, and laparoscopic approaches are generally preferred. ${ }^{178}$ RCG and necrosectomy has been described as a safe alternative, with improved ergonomics facilitating retroperitoneal access, pancreatic debridement, and gastrotomy closure. ${ }^{179,180}$ At UPMC, we retrospectively compared 14 robotic, 6 laparoscopic, and 20 
endoscopic cyst gastrostomies. ${ }^{180}$ Minimally invasive surgical approaches and endoscopic approaches were comparable with respect to mortality, complications, and failure rates. Surgery was associated with a lower reintervention and readmission rate, but longer hospital stay. Total costs (index procedure, hospitalization, readmissions, reinterventions) were similar for both groups.

RCG technique-Initial set up and port placements are similar to RDP, except for not requiring the liver retractor and the $12 \mathrm{~mm}$ assistant port is in the right lower quadrant and the $5 \mathrm{~mm}$ assistant port is in the left lower quadrant. A $5 \mathrm{~cm}$ anterior gastrotomy is made with hook cautery, and laparoscopic ultrasound is used against the posterior wall to localize the WON. Overlying the WON, a $1 \mathrm{~cm}$ posterior gastrotomy is made and the cavity is drained with suction.

Cyst gastrostomy (CG) is performed using 1 of 2 techniques. A linear stapler is used to create the CG and expose the base of the cavity, or alternatively the cavity is opened with cautery and a CG is sewn robotically after debridement (Fig. 19). For the latter approach, the posterior gastrotomy is extended, and 3-0 V-Loc suture is used to create a running CG. Pancreatic debridement is performed through the CG and collected in an Endo Catch bag (Fig. 19). The anterior gastrotomy is closed either with a linear stapler, or with a 2-layer suture closure with inner layer running 3-0 V-Loc suture and outer layer Lembert sutures with 3-0 silk. A cholecystectomy is performed using the same configuration, if indicated.

Summary-Minimally invasive pancreatic surgery presents a technical challenge due to its location and characteristics. The improved ergonomics and optics offered by the robotic platform provide surgeons with additional tools for maximizing the benefits of minimally invasive surgery, while still adhering to the tenets of open surgery. The data to date demonstrate noninferiority of the robotic approach with respect to oncologic and in-hospital outcomes. The data also indicate the presence of a unique learning curve for robotic surgery, emphasizing the need for standardized training curricula and continued assessment of technical performance.

\section{Robotic gastrectomy for gastric adenocarcinoma}

\section{Ashley E. Russo and Vivian E. Strong}

There are nearly 1 million new cases of gastric cancer each year and it remains the third leading cause of cancer related deaths worldwide. ${ }^{181}$ There is wide geographical variation in the incidence of gastric cancer, with the highest incidence of disease seen in Eastern Asia, Eastern Europe, and some Latin American countries. In Western countries, like the United States, the incidence of gastric cancer is much lower, with approximately 25,000 new cases diagnosed annually. Over the last several years, there has been a particular increase in gastroesophageal junction and gastric cardia tumors, likely due to the obesity epidemic and prevalence of gastroesophageal reflux disease, ${ }^{182,183}$ as well as a $70 \%$ increase in noncardia gastric cancer in Caucasian patients in the 25- to 39-year-old age group in the United States. ${ }^{184}$ Although presentation with earlier stage disease is a well-established observation in the East, likely due to higher incidence of disease and more robust screening protocols, the incidence of earlier stage presentation is increasing in the United States as well. Our high- 
volume institution in the United States has observed nearly a doubling in the presentation of early stage disease, from $20 \%$ to $40 \%$, since $1985 .{ }^{185}$ This increase in early stage presentation has given way to the more widespread application and utilization of minimally invasive techniques to treat gastric cancer, as surgery remains the only potentially curative option for patients with gastric cancer.

The use of minimally invasive gastrectomy has been studied in both Eastern and Western countries. The generalizability of these studies, however, is limited by the differences in presentation of gastric cancer in the East and West. Tumors located in the proximal third of the stomach are more common in Western countries and patients typically present with more advanced stage disease often due to lack of government supported screening programs. 182,186-191 Despite geographic differences in presentation, multiple retrospective studies, prospective studies, and meta-analyses from both the East and the West have shown oncologic equivalency between minimally invasive and open gastrectomy for gastric cancer. 192 Many of these studies have highlighted the benefits of minimally invasive procedures which include decreased estimated blood loss, shorter length of hospital stay, more expeditious return of bowel function, lower analgesic requirements, more rapid recovery time, and improved overall quality of life in patients who undergo minimally invasive gastrectomy compared to open gastrectomy. Perhaps the most important observation in the United States has been the more rapid postoperative recovery after minimally invasive gastrectomy, allowing a higher proportion of patients to go on to receive indicated adjuvant systemic therapy rather than being limited by the morbidity of an open operation. ${ }^{193}$

Despite a fairly robust body of data supporting minimally invasive techniques for appropriately selected patients, factors such as learning curves associated with advanced minimally invasive techniques and patient selection particularly early in a surgeon's experience are still extremely important considerations when selecting surgical approach. Since gastric cancer is still rarely seen in the United States outside of high-volume centers, the widespread acceptance of minimally invasive approaches for gastric cancer has been limited by the learning curve associated with both laparoscopic and robotic gastrectomy and the technical skills required to achieve acceptable oncologic outcomes.

Robotic-assisted gastrectomy-The use of the robotic platform for gastric adenocarcinoma was first described in 2003, 194,195 and then later in 2007 in the United States. ${ }^{196}$ Since that time, multiple retrospective series of robotic gastrectomy for gastric cancer have been published. ${ }^{194,197-207}$ The conclusions that can be drawn from these retrospective studies are limited due to great variability in inclusion criteria, surgeon experience, type of reconstruction performed, and the outcomes evaluated. The robotic surgery platform does, however, offer several widely accepted technical advantages over laparoscopy.

In this section, we highlight important considerations regarding patient selection and learning curve, and describe in detail the technical aspects of RG for gastric cancer. Lastly, the current literature on RG for gastric cancer is summarized with a focus on perioperative and long-term outcomes. 
Patient selection-Patient selection, especially early in a surgeon's experience, is paramount. Due to longer operative times compared to both laparoscopic and open resections, surgeons should be especially careful when selecting patients early in their learning curve. ${ }^{194}$ The ideal candidates for robotic-assisted gastrectomy early on are those with minimal medical comorbidities, low or normal BMI, small tumors, distal tumors, and those who have not received neoadjuvant chemotherapy. Patients with a CDH1 mutation are excellent candidates for a robotic approach. $\mathrm{CDH} 1$ positive patients have an approximately $70 \%$ lifetime risk of developing gastric adenocarcinoma, therefore it is recommended that these patients undergo a prophylactic total gastrectomy. ${ }^{204}$ The critical element of a prophylactic total gastrectomy is ensuring that the proximal and distal margins are free of gastric mucosa on frozen section. Although there are no absolute contraindications for robotic-assisted gastrectomy, relative contraindications may include patients with significant intra-abdominal adhesions, diffuse histology, large tumor size, or invasion into adjacent organs. Similar to principles observed with any minimally invasive surgery techniques, dense intra-abdominal adhesions can prevent safe visualization of important structures and therefore compromise the technical performance of the operation.

In patients with diffuse histology, the proximal and distal extents of the tumor cannot always be predicted preoperatively and palpation is typically utilized to determine appropriate resection margins. For these tumors, haptic feedback is essential for determining the proximal and distal extent of the tumor. Although margins are sent for frozen section to confirm cancer-free margins, the inability to confirm the presence of grossly normal tissue via palpation makes robotic resection of diffuse histology more difficult. Robotic surgery also requires institutional support, as successful performance requires coordinated teamwork within the operating room with all team members having familiarity not only with the procedure being performed, but also with the robotic platform. Inclusion criteria may be expanded as surgeon experience with the robotic technique increases.

The learning curve-It is hypothesized that the learning curve for robotic gastrectomy is less than that for laparoscopic gastrectomy due to the ergonomic and technical advantages provided by the robotic platform, especially for surgeons who have experience in advanced laparoscopy. Some of the main advantages of the robotic platform over laparoscopy are that the camera provides 3-dimensional, high definition, stable, and magnified views of the operative field. The robotic instruments are able to articulate and provide $7^{\circ}$ of freedom. Due to enhanced articulation and added degrees of freedom of the robotic instruments, suturing and difficult dissections can be done with relative ease as compared to laparoscopy. Robotic suturing is an effective method for primarily closing a defect of any size or location and is fairly straightforward given the added degrees of freedom provided by the articulating robotic instruments.

Some authors have suggested 20-25 cases for learning robotic gastrectomy by advanced laparoscopic surgeons. ${ }^{198,205}$ A study from 2009 evaluated 60 subtotal gastrectomies with gastro-duodenostomy performed by a single surgeon. ${ }^{206}$ The cases were broken into 3 groups of 20 and represented early laparoscopic, early robotic, and late laparoscopic experience. The study revealed that early robotic cases achieved nearly equivalent outcomes to late laparoscopic cases and that the robotic cases were associated with less blood loss, 
increased lymph node retrieval, shorter hospital stay, and earlier initiation of diet compared to early laparoscopic cases, suggesting that even early on in robotic experience, surgeons with advanced laparoscopic skills can skillfully perform robotic gastrectomies. Kim and colleagues performed a more statistically rigorous and comprehensive multidimensional analysis of the learning curve for 481 laparoscopic vs 172 robotic distal gastrectomy performed by a single surgeon from May 2003 to April 2009. ${ }^{201}$ The authors found that 95 cases were required for learning with robotic gastrectomy, and 270 cases were required for laparoscopic gastrectomy. This study again suggested that experience with laparoscopic surgery may enhance the learning process for robotic gastrectomy since the surgeon had completed 148 laparoscopic gastrectomies before the first robotic case was attempted. More recently, a study evaluated the first 20 consecutive robot-assisted distal gastrectomies performed by 3 experienced laparoscopic surgeons and suggested that amount of laparoscopic experience affected both time to operative time stabilization and reduction in overall operative time and that surgeons with adequate laparoscopic experience can quickly overcome the learning curve for robotic gastrectomy. 207

There have been no studies to date prospectively evaluating learning curves from initial surgeon experience in RG vs LG. It has been suggested that experienced open surgeons can transition directly to the robotic platform without an intermediate laparoscopic step, ${ }^{208}$ but formal simulation training with the robotic platform with both dry and wet labs is imperative. Further-more, one should at least be familiar with laparoscopic exposure of relevant anatomy and with laparoscopic tissue handling while still having haptic feedback, which is lost with the robotic platform.

\section{Technical aspects of robotic gastrectomy}

Patient positioning and port placement: Robotic gastrectomy is performed with the patient in the supine position on a split-leg table (Fig. 20). The patient's arms can either be tucked or positioned on arm boards with adequate padding of elbows and hands to avoid pressure points. The patient is secured to the table at the shoulders using foam blocks and heavy-duty adhesive tape applied circumferentially around the blocks and the table. Fixation is also applied at the hips with a safety belt and circumferentially at the knees. Footboards may also be applied at the feet as a way to avoid sliding during the steep reverse Trendelenburg of at least $45^{\circ}$ necessary for robotic gastrectomy. Once patient positioning is completed, it is important to place the patient in steep reverse Trendelenburg as a test to assure stability prior to starting the operation. Final patient positioning must be achieved prior to docking the robot as further changes in position cannot be made once the robotic arms are in place.

Port placement for RG follows the same principles as port placement for any laparoscopic or robotic procedure, with the placement of the camera port at a distance of $15-20 \mathrm{~cm}$ from the target anatomy, placement of robot ports at least $8 \mathrm{~cm}$ apart from each other, and an assistant port at least $5 \mathrm{~cm}$ from adjacent robotic ports. Although multiple variations of port placement have been described for robotic gastrectomy, the placement illustrated in Fig. 21 is recommended. 
Pneumoperitoneum is established with a Veress needle in the left upper quadrant, just off of the left costal margin. A 10 or $12 \mathrm{~mm}$ trocar is then placed in the midline above or below the umbilicus depending on the patient's body habitus, with a goal of port placement $15-20 \mathrm{~cm}$ from the target anatomy. In the majority of cases, the infraumbilical position is best as it is caudal enough for the omentectomy and construction of the jejunojejunal anastomosis and close enough to the esophageal hiatus for construction of the esophagojejunostomy. Under direct visualization, $28 \mathrm{~mm}$ da Vinci ports are then placed in the left midclavicular line and left anterior axillary line at least $8 \mathrm{~cm}$ from each other and slightly off-set from the plane of the camera port. An additional 10 or $12 \mathrm{~mm}$ port is then placed in the right midclavicular line and a $5 \mathrm{~mm}$ assistant port is placed in the right anterior axillary line, both slightly off-set from the plane of the camera port. A liver retractor is also frequently placed in the left upper quadrant via a small subxiphoid stab incision. This retractor facilitates significant retraction of the left lateral lobe of the liver and exposure of the esophageal hiatus.

After satisfactory placement of all ports, the abdomen is explored for adhesions and evidence of peritoneal disease that would preclude the ability to perform a curative intent resection. During initial abdominal exploration, an attempt it made to identify the location of the tumor from the extraluminal surface of the stomach. If the location of the tumor cannot be determined in this fashion, especially in the case of tumors of the gastroesophageal junction or if a distal subtotal gastrectomy is to be performed, endoscopy should be performed for intraluminal tumor localization. A silk stitch is placed laparoscopically to mark the level of transection of the stomach that will be most likely to achieve a negative proximal margin. After tumor localization occurs and the decision has been made to proceed with robotic resection, the patient is placed in steep reverse Trendelenburg of at least $45^{\circ}$ and the robot is docked. Robot arms 1 and 3 are attached to the 2 left-sided ports and arm 2 is attached to the large 10 or $12 \mathrm{~mm}$ right-sided port in the right midclavicular line. A fenestrated bipolar grasper is placed in arm 2 and a harmonic scalpel or monopolar scissor is placed in arm 1. A grasping forcep, preferably a Cadiere, is placed in arm 3.

\section{Procedural steps}

Omentectomy: The first step of a gastrectomy is to perform an omentectomy. This is accomplished by cephalad retraction of the greater omentum and identification of the transverse colon. The avascular plane is entered between the greater omentum and the transverse colon and the omentum is carefully taken off of the colon using electrocautery, proceeding in the direction of the splenic flexure. Visualization of the posterior wall of the stomach confirms entry into the lesser sac. The posterior wall of the stomach is then grasped by the bedside assistant and is retracted anteriorly and to the patient's right side (Fig. 22). The omentectomy is carried up toward the spleen. In a distal subtotal gastrectomy, the omentectomy is stopped at the edge of the stomach just prior to reaching the short gastric vessels. In a total gastrectomy, the omentectomy is carried up to the esophageal hiatus, and the short gastric vessels are divided under direct visualization using the energy sealant device in arm 1. Once this has been completed, the posterior wall of the stomach is grasped with arm 3 of the robot and is retracted toward the patient's left shoulder. The omentectomy continues toward the hepatic flexure of the colon. Once the omentectomy is complete and 
the omentum is completely removed from its colonic attachments, it can be placed in the left upper quadrant for removal later in the operation.

Dissection of the greater curve of the stomach: Attention is then turned to division of the posterior attachments between the stomach and pancreas. Using either sharp dissection or an energy sealant device, the posterior attachments are divided in the direction of the pylorus. The right gastroepiploic vessels are identified and dissected circumferentially at the level of the anterior border of the pancreas (Fig. 23). The vessels are then divided at their origin from the gastroduodenal vessels with a linear stapler using a vascular load. The stapler is introduced by removing robot arm 2 and the associated port from the $10-12 \mathrm{~mm}$ port site in the right midclavicular line and allowing the bedside assistant to insert the linear stapler into the peritoneal cavity under direct visualization. Alternatively, the right gastroepiploic vessels may be divided using clips instead of a stapler.

Division of the proximal duodenum: Next the pylorus is identified by the vein of Mayo and/or white line, and attention is turned toward the suprapyloric region. The gastrohepatic omentum is incised with hook monopolar electrocautery or a harmonic scalpel in arm 1 . The right gastric artery is identified. Using the harmonic scalpel in arm 1, the right gastric artery is ligated at its base off of the proper hepatic artery. The lymphatic tissue along the proper and common hepatic arteries is swept medially toward the stomach, and a window is created at the level of the pylorus (Fig. 24). Using a combination of blunt dissection and the harmonic scalpel, the posterior aspect of the pylorus and proximal duodenum is elevated off of the retroperitoneum. A linear stapler is once again introduced through the 10-12 mm right midclavicular port site. Loaded with both a blue (bowel) load and bioabsorbable stapler line reinforcement, the proximal duodenum is stapled and divided just distal to the pylorus (Fig. 25).

Lymphadenectomy: After division of the proximal duodenum, the distal stomach is then retracted toward the patient's left shoulder utilizing robot arm 3 in order to prepare for completion of a D2 lymphadenectomy. The lymphadenectomy that was previously started along the proper and common hepatic arteries is then continued along the common hepatic artery toward the celiac axis and proximal splenic artery. During this dissection, the left gastric artery is identified at the celiac axis and is divided at its base with a vascular load of the linear stapler or surgical clips.

Division of the stomach or distal esophagus: Using the harmonic scalpel in arm 1, the gastrohepatic omentum is further incised up to the level of the esophageal hiatus. During a distal subtotal gastrectomy, the level 1 and 2 lymph nodes are peeled off of the proximal stomach down to the level where the stomach will be divided with a linear stapler. During a total gastrectomy, level 1 and 3 lymph nodes are dissected with the proximal stomach up to the right crux of the diaphragm. The peritoneal fat and any fat overlying the esophagus are opened and the distal esophagus is dissected circumferentially. Finally, the distal esophagus is divided with linear stapler loaded with a blue (bowel) load.

Specimen retrieval: Following division of the proximal extent of the gastrectomy specimen, the robotic camera is moved to the right sided 10-12 mm midclavicular port and a specimen 
bag is introduced into the peritoneum via the umbilical port site. The entire specimen is placed in the specimen retrieval bag and is removed via the umbilical port site, which generally needs to be enlarged slightly for specimen retrieval. The proximal margin is marked with a stitch and routinely sent for frozen section margin analysis. During a total gastrectomy, we do not routinely send the distal margin for frozen section analysis unless the operation is a prophylactic total gastrectomy for hereditary diffuse gastric cancer. In the case of hereditary diffuse gastric cancer, the frozen section must reveal normal esophageal mucosa at the proximal margin and normal duodenal mucosa at the distal margin. In distal gastrectomies with tumors located near the pylorus, the surgeon may choose to send frozen section analysis of the distal margin. Following specimen retrieval, the camera is returned to its normal position at the umbilical port site and attention is then turned to the reconstruction.

Gastrointestinal reconstruction: For a distal subtotal gastrectomy in which no more than one half of the stomach was removed, our preferred method of reconstruction is an antecolic, Billroth II reconstruction. If more than one half of the stomach is removed or if a total gastrectomy is performed, our preferred method of reconstruction is Roux-Y gastrojejunostomy or esophagojejunostomy, respectively. The first step of all reconstructions is cephalad retraction of the colon in order to identify the ligament of Treitz. A mobile piece of jejunum approximately $30-40 \mathrm{~cm}$ downstream from the ligament of Treitz is identified and divided with a linear stapler. For a Billroth II or Roux-Y gastrojejunostomy, a side-toside stapled gastrojejunostomy is created with a $60 \mathrm{~mm}$ laparoscopic stapler. The enterotomy is closed with a running 3-0 silk suture using needle drivers in robot arms 1 and 2 (Fig. 26).

For an esophagojejunostomy, an end-to-side anastomosis is created between the esophagus and the roux limb using a circular stapler or alternatively, a linear stapled anastomosis. In order to create the circular stapled anastomosis, a transoral anvil (OrVil, Covidien; Fig. $27)^{209}$ is passed on a nasogastric tube by the anesthesiologist and passed until the tip of the nasogastric tube reaches the stapled end of the esophagus. A small esophagotomy is made using electrocautery to allow for the tube to be gently pulled through the distal esophagus into the peritoneal cavity. The nasogastric tube is then gently detached from the anvil and the tube is removed through the $10-12 \mathrm{~mm}$ port in the right midclavicular line. Using the harmonic scalpel in arm 1, the staple line of the roux limb is removed and the other end of the circular stapler is inserted into the proximal end of the Roux limb. The circular stapler is fired. Visual inspection and the confirmation of 2 intact donuts confirm a successful anastomosis. The open end of the Roux limb is closed with a linear stapler (Fig. 28). The donuts are sent to pathology for examination, as they represent the true final esophageal margin. For Roux-en-Y reconstructions, a side-to-side stapled jejunojejunostomy is then created approximately $60-70 \mathrm{~cm}$ downstream from the proximal anastomosis, and the common enterotomy is closed with a running 2-0 silk suture. Mesenteric defects from the jejunojejunostomy and Petersen's space are closed with running 3-0 Vicryl sutures. ${ }^{210}$ After careful inspection of the abdomen for adequate hemostasis, the robot is undocked, the patient is flattened out, all port sites are closed, and the procedure is completed. 
Perioperative outcomes-There have been multiple nonrandomized retrospective studies that have been done to evaluate the perioperative outcomes and long-term oncologic outcomes associated with robotic gastrectomy as compared to both open gastrectomy and laparoscopic gastrectomy. ${ }^{194-196,200,211-216}$ Findings from many of the major studies are summarized in Table 2 and focus primarily on short-term outcomes, namely operative time, estimated blood loss, lymph node retrieval, conversion rate, morbidity rate, and mortality rate between the different operative approaches.

Short term outcomes: The largest single-center study to date was done by Kim and colleagues in 2012 and included 5839 patients who underwent open $(\mathrm{n}=4542)$, laparoscopic $(n=861)$, or robotic $(n=436)$ gastrectomy for gastric adenocarcinoma. ${ }^{202}$ The study found no differences in overall morbidity or mortality among the groups. There were, however, differences found in the type of complications associated with the different operative approaches. The open approach was associated with more postoperative bowel obstruction, ileus, and abscess formation, while the laparoscopic and robotic approaches were associated with a higher incidence of anastomotic leak. A 2013 meta-analysis compared 7 studies which included 1967 patients who underwent either robotic $(n=404)$, open $(n=718)$, or laparoscopic $(\mathrm{n}=845)$ gastrectomy and compared short-term outcomes between the various operative approaches. ${ }^{217}$ Robotic gastrectomy was associated with shorter hospital stay compared to open gastrectomy and significantly less blood loss compared to laparoscopic gastrectomy, however operative time was longer in the robotic group compared to both the open and laparoscopic groups. There were no significant differences in lymph node retrieval or overall complication rates.

Several meta-analyses have also compared differences in short-term outcomes between robotic gastrectomy and laparoscopic gastrectomy alone. A meta-analysis by Liao and colleagues included 2235 patients with gastric cancer, 1473 patients in the laparoscopic group and 762 patients in the robotic group. ${ }^{218}$ The robotic approach was associated with longer operative time but less blood loss. There were no significant differences in length of hospital stay, postoperative complication rate, proximal or distal margin positivity rates, lymph node retrieval, postoperative complications, or mortality. Another meta-analysis from 2012 included 918 patients, 268 in the robotic group and 650 in the laparoscopic group. ${ }^{219}$ Robotic gastrectomy was associated with longer operative time but less blood loss. There were no differences in lymph node retrieval, overall morbidity, perioperative mortality rates, or length of hospital stay. Finally, Xiong and colleagues compared short-term outcomes in 2495 patients, 736 in the robotic group, and 1759 in the laparoscopic group. ${ }^{220}$ The study found less intraoperative blood loss and earlier initiation of oral intake with robotic gastrectomy, but did show longer operative time and shorter distal resection margin. There were no differences in lymph node retrieval, proximal resection margin, conversion rates, length of hospital stay, morbidity, or mortality.

Long-term outcomes: Although most studies to date have focused on the short-term outcomes as presented above, some limited data on long-term outcomes has begun to emerge more recently in the literature as adequate follow-up time is reached on patients who have undergone robotic gastrectomy in the past. A single-center study from 2016 compared 
long-term outcomes of robotic gastrectomy compared to laparoscopic gastrectomy for gastric cancer. ${ }^{221}$ This study evaluated 521 patients who underwent gastrectomy from 2009 to 2012. The long-term outcomes evaluated were 3-year overall survival and 3-year recurrence free survival. The study found no significant differences in 3-year overall survival (RG, $86.9 \%$ vs $88.8 \%, P=0.636$ ) or 3 -year recurrence free survival (RG, $86.9 \%$ vs LG, $86.3 \% ; P=0.905)$. The authors also compared short-term and long-term outcomes between surgeons with extensive minimally invasive surgery experience and those without and found that long-term oncologic outcomes and short-term complications were equivalent between the 2 groups.

Summary-Although minimally invasive techniques are widely accepted in the treatment of gastric adenocarcinoma, robotic gastrectomy is now emerging as a preferred surgical approach over laparoscopy in some centers. Patient selection is of paramount importance when it comes to deciding which patients are appropriate for robotic gastrectomy, particularly early in a surgeon's learning curve. Studies have suggested, however, that surgeons with experience in advanced laparoscopy may be able to overcome the learning curve more rapidly. The technical advantages conferred by the robotic platform allow for more precise dissection, enhanced lymph node retrieval, better 3D visualization, and less blood loss as compared to traditional laparoscopy, although these advantages do come at the cost of longer operative times. From a patient perspective, robotic gastrectomy has been associated with earlier initiation of diet and shortened length of hospital stay as compared to open approaches. Morbidity and mortality rates have been shown to be equivalent in the literature thus far, owning to the apparent surgical and oncologic adequacy of the robotic approach. Long-term outcome data are limited but suggest acceptable survival and recurrence rates at this time. Additional studies on long-term outcomes are needed to fully appreciate the clinical benefit of the robotic approach, particularly as they relate to any benefits seen in recurrence free survival as a result of the ability to perform a more precise lymphadenectomy. As surgeons gain more experience with the robotic platform, it is likely that we will see expanded inclusion criteria with respect to patient selection, including patients with more advanced disease and higher BMI, and the role of robotic gastrectomy in the treatment of gastric adenocarcinoma in these situations is yet to be determined.

\section{Biography}

Seth Felder, MD, is Assistant Attending Surgeon in the Department of Gastrointestinal Surgery at Moffitt Cancer Center. After receiving his medical degree at the University of South Florida, he completed a general surgery residency at Cedars-Sinai Medical Center, a colorectal surgery fellowship at the University of Minnesota, and a colorectal surgical oncology fellowship at Memorial Sloan Kettering Cancer Center.

Rajesh Ramanathan, MD, is a clinical fellow in complex general surgical oncology at the University of Pittsburgh Medical Center. He attended Dartmouth Medical School. He completed his general surgery residency and research fellowship at Virginia Commonwealth University Medical Center. He has a clinical interest in gastrointestinal and hepatobiliary surgery. 
Ashley Russo, MD, is a surgical outcomes research fellow at Memorial Sloan Kettering Cancer Center. She received her medical degree from the University of Vermont College of Medicine. She began her residency in general surgery at the University of Massachusetts Medical Center, where she will return to finish residency following her 2-year dedicated research fellowship, with plans to pursue a surgical oncology fellowship after residency. Her research interests include clinical and translational research in gastroesophageal junction, gastric, and adrenal cancer.

Rosa Maria Jimenez-Rodriguez, MD, PhD, is a Research Fellow in the Colorectal Service, Department of Surgery at Memorial Sloan Kettering Cancer Center. She received her medical degree and completed a residency in general surgery at University Hospital Virgen del Rocio in Sevilla, Spain where she also received her PhD at the University of Sevilla, Spain.

Melissa Hogg, MD, MS, is Assistant Professor in the Division of Surgical Oncology at the University of Pittsburgh Medical Center. She completed her medical degree and general surgery residency at Northwestern University. She then joined University of Pittsburgh for a fellowship in complex general surgical oncology and master's degrees in clinical research and medical education. Her clinical interests include pancreaticobiliary surgery and minimally invasive and robotic surgery. Her research interests include surgical education and clinical outcomes research.

Amer Zureikat, MD, is Assistant Professor in the Division of Surgical Oncology, and CoDirector of the UPMC Pancreatic Cancer Center at the University of Pittsburgh Medical Center. He completed his medical degree at the Royal College of Surgeons, and general surgery residency at the University of Chicago. He joined the University of Pittsburgh for a fellowship in complex general surgical oncology. His clinical interests include pancreaticobiliary surgery and minimally invasive and robotic surgery. His research interests include clinical outcomes research and biomarkers in pancreatic cancer.

Vivian Strong, MD, is an attending surgeon at Memorial Sloan Kettering Cancer Center and Associate Professor in Surgery at Weill Cornell Medical College. She received her medical degree from Weill Cornell Medical College, where she also completed her general surgery residency. She completed her advanced laparoscopic fellowship at Cornell/Columbia. She specializes in minimally invasive and open resections for patients with gastric and adrenal tumors. Her research focuses on clinical and translational research in gastric and adrenal tumors and minimally invasive approaches.

Herbert Zeh, III, MD, is Professor and Chair in the Division of GI Surgical Oncology at the University of Pittsburgh Medical Center, as well as Associate Director of UPMC Hillman Cancer Center, and Senior Director of UPMC Surgical Services. He completed his medical degree at the University of Pittsburgh and a Medical Staff Fellowship at the National Cancer Institute Surgery Branch. He joined the Johns Hopkins Hospital for general surgery residency and surgical oncology fellowship. His clinical interests include pancreatic surgery and robotic, minimally invasive surgery. His research interests include the role of 
damage-associated molecular pattern molecules (DAMPs) and advanced glycation end product-specific receptor (RAGE) in pancreatic cancer.

Martin R. Weiser, MD, is Stuart Quan Chair in Colorectal Surgery and Vice Chair for Education and Faculty Development in the Department of Surgery at Memorial Sloan Kettering Cancer Center. He received his medical degree from the University of Chicago, completed a residency in general surgery at Brigham and Women's Hospital, a fellowship in colon and rectal surgery at Mount Sinai Medical Center, and a fellowship in surgical oncology at Memorial Sloan Kettering.

\section{References}

1. Peterson CY, Weiser MR. Robotic colorectal surgery. J Gastrointest Surg. 2014;18:398-403. [PubMed: 23949476]

2. Smith JJ, Weiser MR. Outcomes in non-metastatic colorectal cancer. J Surg Oncol. 2014;110:518526. [PubMed: 24962603]

3. Intuitive Surgical Inc. Intuitive Surgical's EndoWrist Instruments http://www.intuitivesurgicalcom/ products/instruments, Date Accessed October 1, 2017.

4. Pappou EP, Weiser MR. Robotic colonic resection. J Surg Oncol. 2015;112:315-320. [PubMed: 26179217]

5. Baek SJ, Kim CH, Cho MS, et al. Robotic surgery for rectal cancer can overcome difficulties associated with pelvic anatomy. Surg Endosc. 2015;29:1419-1424. [PubMed: 25159651]

6. Moorthy K, Munz Y, Dosis A, et al. Dexterity enhancement with robotic surgery. Surg Endosc. 2004;18:790-795. [PubMed: 15216862]

7. Jafari M, Rivadeneira DE, Pigazzi A. Robotic Surgery Minimally Invasive Approaches to Colon and Rectal Disease: Technique and Best Practices. New York: Springer; 2015:273-282.

8. Weber PA, Merola S, Wasielewski A, Ballantyne GH. Telerobotic-assisted laparoscopic right and sigmoid colectomies for benign disease. Dis Colon Rectum. 2002;45:1689-1694 discussion 95-6. [PubMed: 12473897]

9. Delaney CP, Lynch AC, Senagore AJ, Fazio VW. Comparison of robotically performed and traditional laparoscopic colorectal surgery. Dis Colon Rectum. 2003;46:1633-1639. [PubMed: 14668588]

10. Pigazzi A, Ellenhorn JD, Ballantyne GH, Paz IB. Robotic-assisted laparoscopic low anterior resection with total mesorectal excision for rectal cancer. Surg Endosc. 2006;20:1521-1525. [PubMed: 16897284]

11. Cadiere GB, Himpens J, Germay O, et al. Feasibility of robotic laparoscopic surgery: 146 cases. World J Surg. 2001;25:1467-1477. [PubMed: 11760751]

12. Halabi WJ, Kang CY, Jafari MD, et al. Robotic-assisted colorectal surgery in the United States: a nationwide analysis of trends and outcomes. World J Surg. 2013;37:2782-2790. [PubMed: 23564216]

13. Midura EF, Hanseman DJ, Hoehn RS, et al. The effect of surgical approach on short-term oncologic outcomes in rectal cancer surgery. Surgery. 2015;158:453-459. [PubMed: 25999253]

14. Barbash GI, Glied SA. New technology and health care costs-the case of robot-assisted surgery. N Engl J Med. 2010;363:701-704. [PubMed: 20818872]

15. Turchetti G, Palla I, Pierotti F, Cuschieri A. Economic evaluation of da Vinci-assisted robotic surgery: a systematic review. Surg Endosc. 2012;26:598-606. [PubMed: 21993935]

16. Smith JJ, Feo L, Garcia-Aguilar J. Totally robotic low anterior resection In: Kim J, Garcia-Aguilar Julio, eds. Surgery for Cancers of the Gastrointestinal Tract: A Step-by-Step Approach. New York: Springer; 2015:237-245.

17. Tyler JA, Fox JP, Desai MM, Perry WB, Glasgow SC. Outcomes and costs associated with robotic colectomy in the minimally invasive era. Dis Colon Rectum. 2013;56:458-466. [PubMed: 23478613] 
18. Juo YY, Hyder O, Haider AH, Camp M, Lidor A, Ahuja N. Is minimally invasive colon resection better than traditional approaches? First comprehensive national examination with propensity score matching. JAMA Surg. 2014;149:177-184. [PubMed: 24352653]

19. Wormer BA, Dacey KT, Williams KB, et al. The first nationwide evaluation of robotic general surgery: a regionalized, small but safe start. Surg Endosc. 2014;28:767-776. [PubMed: 24196549]

20. Davis BR, Yoo AC, Moore M, Gunnarsson C. Robotic-assisted versus laparoscopic colectomy: cost and clinical outcomes. JSLS. 2014;18:211-224. [PubMed: 24960484]

21. Delaney CP, Senagore AJ, Ponsky L. Robot-assisted surgery and health care costs. N Engl J Med. 2010;363:2175 author reply 6.

22. Keller DS, Hashemi L, Lu M, Delaney CP. Short-term outcomes for robotic colorectal surgery by provider volume. J Am Coll Surg. 2013;217:1063-1069 e1. [PubMed: 24041555]

23. Villamere J, Gebhart A, Vu S, Nguyen NT. Utilization and outcome of laparoscopic versus robotic general and bariatric surgical procedures at Academic Medical Centers. Surg Endosc. 2015;29:1729-1736. [PubMed: 25318362]

24. Liberman D, Trinh QD, Jeldres C, Zorn KC. Is robotic surgery cost-effective: yes. Curr Opin Urol. 2012;22: 61-65 [PubMed: 22037320]

25. Collinson FJ, Jayne DG, Pigazzi A, et al. An international, multicentre, prospective, randomised, controlled, unblinded, parallel-group trial of robotic-assisted versus standard laparoscopic surgery for the curative treatment of rectal cancer. Int J Colorectal Dis. 2012;27:233-241. [PubMed: 21912876]

26. Jayne D, Pigazzi A, Marshall H, et al. Effect of robotic-assisted vs conventional laparoscopic surgery on risk of conversion to open laparotomy among patients undergoing resection for rectal cancer: the ROLARR randomized clinical trial. JAMA. 2017;318:1569-1580. [PubMed: 29067426]

27. Xu J, Wei Y, Ren L, Feng Q, Chen J, Zhu D. Robot-assisted vs. laparoscopic vs. open abdominoperineal resections for low rectal cancer: short-term outcomes of a single-center prospective randomized controlled trial. Eur Soc Med Oncol Congr. 2017:482PD.

28. A Trial to Assess Robot-assisted Surgery and Laparoscopy-assisted Surgery in Patients with Mid or Low Rectal Cancer (COLRAR). Kyungpook National University; 2011.

29. D'Annibale A OC. Fiscon V, Trevisan P. et al. Videolaparoscopic surgery in the treatment of colorectal disease: our experience with 200 patients. Chir Ital. 2002;54:777-783. [PubMed: 12613325]

30. Braumann C, Jacobi CA, Menenakos C, Borchert U, Rueckert JC, Mueller JM. Computer-assisted laparoscopic colon resection with the Da Vinci system: our first experiences. Dis Colon Rectum. 2005;48:1820-1827. [PubMed: 16142433]

31. Rawlings AL, Woodland JH, Crawford DL. Telerobotic surgery for right and sigmoid colectomies: 30 consecutive cases. Surg Endosc. 2006;20:1713-1718. [PubMed: 17008953]

32. Hellan M AC, Ellenhorn JD, Paz B, Pigazzi A. Short-term outcomes after robotic-assisted total mesorectal excision for rectal cancer. Ann Surg Oncol. 2007;14:3168-3173. [PubMed: 17763911]

33. Spinoglio G, Summa M, Priora F, Quarati R, Testa S. Robotic colorectal surgery: first 50 cases experience. Dis Colon Rectum. 2008;51:1627-1632. [PubMed: 18484134]

34. Baik SH, Kim NK, Lee KY, et al. Factors influencing pathologic results after total mesorectal excision for rectal cancer: analysis of consecutive 100 cases. Ann Surg Oncol. 2008;15:721-728. [PubMed: 18058183]

35. Trastulli S, Coratti A, Guarino S, et al. Robotic right colectomy with intracorporeal anastomosis compared with laparoscopic right colectomy with extracorporeal and intracorporeal anastomosis: a retrospective multicentre study. Surg Endosc. 2015;29:1512-1521. [PubMed: 25303905]

36. Sujatha-Bhaskar S, Jafari MD, et al. Defining the role of minimally invasive proctectomy for locally advanced rectal adenocarcinoma. Ann Surg. 2017;266:574-581. [PubMed: 28650357]

37. Barrie J, Jayne DG, Wright J, Murray CJ, Collinson FJ, Pavitt SH. Attaining surgical competency and its implications in surgical clinical trial design: a systematic review of the learning curve in laparoscopic and robot-assisted laparoscopic colorectal cancer surgery. Ann Surg Oncol. 2014;21:829-840. [PubMed: 24217787] 
38. Damle RN, Macomber CW, Flahive JM, et al. Surgeon volume and elective resection for colon cancer: an analysis of outcomes and use of laparoscopy. J Am Coll Surg. 2014;218:1223-1230. [PubMed: 24768291]

39. Tam MS, Kaoutzanis C, Mullard AJ, et al. A population-based study comparing laparoscopic and robotic outcomes in colorectal surgery. Surg Endosc. 2016;30:455-463. [PubMed: 25894448]

40. Kuhry E, Bonjer HJ, Haglind E, et al. Impact of hospital case volume on short-term outcome after laparoscopic operation for colonic cancer. Surg Endosc. 2005;19:687-692. [PubMed: 15798899]

41. Guillou PJ, Quirke P, Thorpe H, et al. Short-term endpoints of conventional versus laparoscopicassisted surgery in patients with colorectal cancer (MRC CLASICC trial): multicentre, randomised controlled trial. Lancet. 2005;365:1718-1726. [PubMed: 15894098]

42. Moghadamyeghaneh Z, Carmichael JC, Mills S, Pigazzi A, Nguyen NT, Stamos MJ. Variations in laparoscopic colectomy utilization in the United States. Dis Colon Rectum. 2015;58:950-956. [PubMed: 26347967]

43. Yeo H, Niland J, Milne D, et al. Incidence of minimally invasive colorectal cancer surgery at National Comprehensive Cancer Network centers. J Natl Cancer Inst. 2015;107:362. [PubMed: 25527640]

44. Yeo HL, Isaacs AJ, Abelson JS, Milsom JW, Sedrakyan A. Comparison of open, laparoscopic, and robotic colectomies using a large national database: outcomes and trends related to surgery center volume. Dis Colon Rectum. 2016;59:535-542. [PubMed: 27145311]

45. Bertelsen CA. Complete mesocolic excision an assessment of feasibility and outcome. Dan Med J. 2017;64:1-24.

46. Hohenberger W, Weber K, Matzel K, Papadopoulos T, Merkel S. Standardized surgery for colonic cancer: complete mesocolic excision and central ligation-technical notes and outcome. Colorectal Dis. 2009;11:354-364 discussion 64-5. [PubMed: 19016817]

47. West NP, Hohenberger W, Finan PJ, Quirke P. Mesocolic plane surgery: an old but forgotten technique? Colorectal Dis. 2009;11:988-989. [PubMed: 19558592]

48. Xu H, Li J, Sun Y, et al. Robotic versus laparoscopic right colectomy: a meta-analysis. World J Surg Oncol. 2014;12:274. [PubMed: 25169141]

49. Rawlings AL, Woodland JH, Vegunta RK, Crawford DL. Robotic versus laparoscopic colectomy. Surg Endosc. 2007;21:1701-1708. [PubMed: 17353988]

50. Morpurgo E, Contardo T, Molaro R, Zerbinati A, Orsini C, D’Annibale A. Robotic-assisted intracorporeal anastomosis versus extracorporeal anastomosis in laparoscopic right hemicolectomy for cancer: a case control study. J Laparoendosc Adv Surg Tech A. 2013;23:414-417. [PubMed: 23627922]

51. deSouza AL, Prasad LM, Park JJ, Marecik SJ, Blumetti J, Abcarian H. Robotic assistance in right hemicolectomy: is there a role? Dis Colon Rectum. 2010;53:1000-1006. [PubMed: 20551751]

52. Deutsch GB, Sathyanarayana SA, Gunabushanam V, et al. Robotic vs. laparoscopic colorectal surgery: an institutional experience. Surg Endosc. 2012;26:956-963. [PubMed: 22044968]

53. Park JS, Choi GS, Park SY, Kim HJ, Ryuk JP. Randomized clinical trial of robot-assisted versus standard laparoscopic right colectomy. Br J Surg. 2012;99:1219-1226. [PubMed: 22864881]

54. Lujan HJ, Maciel VH, Romero R, Plasencia G. Laparoscopic versus robotic right colectomy: a single surgeon's experience. J Robot Surg. 2013;7:95-102. [PubMed: 27000901]

55. Casillas MA, Jr, Leichtle SW, Wahl WL, et al. Improved perioperative and short-term outcomes of robotic versus conventional laparoscopic colorectal operations. Am J Surg. 2014;208:33-40. [PubMed: 24239530]

56. Luca F, Ghezzi TL, Valvo M, et al. Surgical and pathological outcomes after right hemicolectomy: case-matched study comparing robotic and open surgery. Int J Med Robot, 2011;7:298-303. [PubMed: 21563286]

57. Gossedge G, Jayne D, Robotic technique for right colectomy In: Kim J, Garcia-Aguilar J, eds. Surgery for Cancers of the Gastrointestinal Tract: A Step-by-Step Approach, New York: Springer; 2015:187-194

58. Agha A, Furst A, Iesalnieks I, et al. Conversion rate in 300 laparoscopic rectal resections and its influence on morbidity and oncological outcome. Int J Colorectal Dis. 2008;23:409-417.

[PubMed: 18185938] 
59. Yamamoto S, Fukunaga M, Miyajima N, et al. Impact of conversion on surgical outcomes after laparoscopic operation for rectal carcinoma: a retrospective study of 1,073 patients. J Am Coll Surg. 2009;208: 383-389. [PubMed: 19318000]

60. Rottoli M, Stocchi L, Geisler DP, Kiran RP. Laparoscopic colorectal resection for cancer: effects of conversion on long-term oncologic outcomes. Surg Endosc. 2012;26:1971-1976. [PubMed: 22237758]

61. Allaix ME, Furnee EJ, Mistrangelo M, Arezzo A, Morino M. Conversion of laparoscopic colorectal resection for cancer: what is the impact on short-term outcomes and survival? World J Gastroenterol. 2016;22: 8304-8313. [PubMed: 27729737]

62. Kim CW, Kim CH, Baik SH. Outcomes of robotic-assisted colorectal surgery compared with laparoscopic and open surgery: a systematic review. J Gastrointest Surg. 2014;18:816-830. [PubMed: 24496745]

63. Feroci F, Lenzi E, Garzi A, Vannucchi A, Cantafio S, Scatizzi M. Intracorporeal versus extracorporeal anastomosis after laparoscopic right hemicolectomy for cancer: a systematic review and meta-analysis. Int J Colorectal Dis. 2013;28:1177-1186. [PubMed: 23371336]

64. Cirocchi R, Trastulli S, Farinella E, et al. Intracorporeal versus extracorporeal anastomosis during laparoscopic right hemicolectomy - systematic review and meta-analysis. Surg Oncol. 2013;22:113. [PubMed: 23116767]

65. Milone M, Elmore U, Di Salvo E, et al. Intracorporeal versus extracorporeal anastomosis. Results from a multicentre comparative study on 512 right-sided colorectal cancers. Surg Endosc. 2015;29:2314-2320. [PubMed: 25414066]

66. Samia H, Lawrence J, Nobel T, Stein S, Champagne BJ, Delaney CP. Extraction site location and incisional hernias after laparoscopic colorectal surgery: should we be avoiding the midline? Am J Surg. 2013;205:264-267 discussion 8. [PubMed: 23375702]

67. Lee L, Mappin-Kasirer B, Sender Liberman A, et al. High incidence of symptomatic incisional hernia after midline extraction in laparoscopic colon resection. Surg Endosc. 2012;26:3180-3185. [PubMed: 22580878]

68. Widmar M, Keskin M, Beltran P, et al. Incisional hernias after laparoscopic and robotic right colectomy. Hernia. 2016;20:723-728. [PubMed: 27469592]

69. van Oostendorp S, Elfrink A, et al. Intracorporeal versus extracorporeal anastomosis in right hemicolectomy: a systematic review and meta-analysis. Surg Endosc. 2017;31:64-77. [PubMed: 27287905]

70. Lujan HJ, Plasencia G, Rivera BX, et al. Advantages of robotic right colectomy with intracorporeal anastomosis. Surg Laparosc Endosc Percutan Tech. 2017;28:36-41.

71. Luca F, Cenciarelli S, Valvo M, et al. Full robotic left colon and rectal cancer resection: technique and early outcome. Ann Surg Oncol. 2009;16:1274-1278. [PubMed: 19242762]

72. Sammour T, Malakorn S, Bednarski BK, et al. Oncological outcomes after robotic proctectomy for rectal cancer: analysis of a prospective database. Ann Surg. 2018;267:521-526. [PubMed: 27997470]

73. Delaney C, Ponsky J, Russ A. Laparoscopic technique for right colectomy In: Kim J, GarciaAguilar J, eds. Surgery for Cancers of the Gastrointestinal Tract: A Step-by-Step Approach. New York: Springer; 2015:175-185.

74. Smith JJ, Garcia-Aguilar J. Advances and challenges in treatment of locally advanced rectal cancer. J Clin Oncol, 2015;33:1797-1808. [PubMed: 25918296]

75. Heald RJ. Total mesorectal excision. Acta Chir Iugosl. 1998;45:37-38. [PubMed: 10951785]

76. Heald RJ, Moran BJ, Ryall RD, Sexton R, MacFarlane JK. Rectal cancer: the Basingstoke experience of total mesorectal excision, 1978-1997. Arch Surg. 1998;133:894-899. [PubMed: 9711965]

77. Champagne BJ, Makhija R. Minimally invasive surgery for rectal cancer: are we there yet? World J Gastroenterol, 2011;17:862-866. [PubMed: 21412496]

78. Kayano H, Okuda J, Tanaka K, Kondo K, Tanigawa N. Evaluation of the learning curve in laparoscopic low anterior resection for rectal cancer. Surg Endosc. 2011;25:2972-2979. [PubMed: 21512883] 
79. Green BL, Marshall HC, Collinson F, et al. Long-term follow-up of the Medical Research Council CLASICC trial of conventional versus laparoscopically assisted resection in colorectal cancer. $\mathrm{Br} \mathrm{J}$ Surg. 2013;100:75-82. [PubMed: 23132548]

80. Bonjer HJ, Deijen CL, Abis GA, et al. A randomized trial of laparoscopic versus open surgery for rectal cancer. N Engl J Med. 2015;372:1324-1332. [PubMed: 25830422]

81. Jeong SY, Park JW, Nam BH, et al. Open versus laparoscopic surgery for mid-rectal or low-rectal cancer after neoadjuvant chemoradiotherapy (COREAN trial): survival outcomes of an open-label, non-inferiority, randomised controlled trial. Lancet Oncol. 2014;15:767-774. [PubMed: 24837215]

82. Stevenson AR, Solomon MJ, et al. Effect of laparoscopic-assisted resection vs open resection on pathological outcomes in rectal cancer: the ALaCaRT randomized clinical trial. JAMA. 2015;314:1356-1363. [PubMed: 26441180]

83. Fleshman J, Branda M, Sargent DJ, et al. Effect of laparoscopic-assisted resection vs open resection of stage II or III rectal cancer on pathologic outcomes: the ACOSOG Z6051 randomized clinical trial. JAMA, 2015;314: 1346-1355. [PubMed: 26441179]

84. Ng SS, Lee JF, Yiu RY, et al. Long-term oncologic outcomes of laparoscopic versus open surgery for rectal cancer: a pooled analysis of 3 randomized controlled trials. Ann Surg. 2014;259:139_ 147. [PubMed: 23598381]

85. Milsom JW, de Oliveira O, Jr, Trencheva KI, Pandey S, Lee SW, Sonoda T. Long-term outcomes of patients undergoing curative laparoscopic surgery for mid and low rectal cancer. Dis Colon Rectum. 2009;52:1215-1222. [PubMed: 19571696]

86. Baek SK, Carmichael JC, Pigazzi A. Robotic surgery: colon and rectum. Cancer J. 2013;19:140146. [PubMed: 23528722]

87. Wexner SD, Bergamaschi R, Lacy A, et al. The current status of robotic pelvic surgery: results of a multinational interdisciplinary consensus conference. Surg Endosc. 2009;23:438-443. [PubMed: 19037694]

88. Trakarnsanga A, Weiser MR. Minimally invasive surgery of rectal cancer: current evidence and options. Am Soc Clin Oncol Educ Book. 2012;2012:214-218.

89. Park JS, Choi GS, Lim KH, Jang YS, Jun SH. S052: a comparison of robot-assisted, laparoscopic, and open surgery in the treatment of rectal cancer. Surg Endosc. 2011;25:240-248. [PubMed: 20552367]

90. D’Annibale A, Pernazza G, Monsellato I, et al. Total mesorectal excision: a comparison of oncological and functional outcomes between robotic and laparoscopic surgery for rectal cancer. Surg Endosc. 2013;27:1887-1895. [PubMed: 23292566]

91. Ielpo B, Caruso R, Quijano Y, et al. Robotic versus laparoscopic rectal resection: is there any real difference? A comparative single center study. Int J Med Robot. 2014;10:300-305. [PubMed: 24692203]

92. Cho MS, Baek SJ, Hur H, et al. Short and long-term outcomes of robotic versus laparoscopic total mesorectal excision for rectal cancer: a case-matched retrospective study. Medicine. 2015;94:e522. [PubMed: 25789947]

93. Park JS, Kim NK, Kim SH, et al. Multicentre study of robotic intersphincteric resection for low rectal cancer. Br J Surg. 2015;102:1567-1573. [PubMed: 26312601]

94. Yamaguchi T, Kinugasa Y, Shiomi A, Tomioka H, Kagawa H, Yamakawa Y. Robotic-assisted vs. conventional laparoscopic surgery for rectal cancer: short-term outcomes at a single center. Surg Today. 2016;46:957-962. [PubMed: 26482845]

95. Trastulli S, Farinella E, Cirocchi R, et al. Robotic resection compared with laparoscopic rectal resection for cancer: systematic review and meta-analysis of short-term outcome. Colorectal Dis. 2012; 14:e134-e156. [PubMed: 22151033]

96. Yang Y, Wang F, Zhang P, et al. Robot-assisted versus conventional laparoscopic surgery for colorectal disease, focusing on rectal cancer: a meta-analysis. Ann Surg Oncol. 2012;19:37273736. [PubMed: 22752371]

97. Memon S, Heriot AG, Murphy DG, Bressel M, Lynch AC. Robotic versus laparoscopic proctectomy for rectal cancer: a meta-analysis. Ann Surg Oncol. 2012;19:2095-2101. [PubMed: 22350601] 
98. Lin S, Jiang HG, Chen ZH, Zhou SY, Liu XS, Yu JR. Meta-analysis of robotic and laparoscopic surgery for treatment of rectal cancer. World J Gastroenterol. 2011;17:5214-5220. [PubMed: 22215947]

99. Lorenzon L, Bini F, Balducci G, Ferri M, Salvi PF, Marinozzi F. Laparoscopic versus roboticassisted colectomy and rectal resection: a systematic review and meta-analysis. Int J Colorectal Dis. 2016;31:161-173. [PubMed: 26410261]

100. Xiong B, Ma L, Huang W, Zhao Q, Cheng Y, Liu J. Robotic versus laparoscopic total mesorectal excision for rectal cancer: a meta-analysis of eight studies. J Gastrointest Surg. 2015;19:516-526. [PubMed: 25394387]

101. Lee SH, Lim S, Kim JH, Lee KY. Robotic versus conventional laparoscopic surgery for rectal cancer: systematic review and meta-analysis. Ann Surg Treat Res. 2015;89:190-201. [PubMed: 26448918]

102. Sun Z, Kim J, Adam MA, et al. Minimally invasive versus open low anterior resection: equivalent survival in a national analysis of 14,033 patients with rectal cancer. Ann Surg. 2016;263:11521158. [PubMed: 26501702]

103. Speicher PJ, Englum BR, Ganapathi AM, Nussbaum DP, Mantyh CR, Migaly J. Robotic low anterior resection for rectal cancer: a national perspective on short-term oncologic outcomes. Ann Surg. 2015;262:1040-1045. [PubMed: 25405559]

104. Roxburgh C, Weiser MR. Difficult Decisions in Colorectal Surgery: An Evidence-Based Approach Laparoscopic versus robotic versus open surgery for rectal cancer. Switzerland: Springer International; 2016.

105. Baik SH, Kwon HY, Kim JS, et al. Robotic versus laparoscopic low anterior resection of rectal cancer: short-term outcome of a prospective comparative study. Ann Surg Oncol. 2009;16:1480 1487. [PubMed: 19290486]

106. Kim J, Baek SJ, Kang DW, et al. Robotic resection is a good prognostic factor in rectal cancer compared with laparoscopic resection: long-term survival analysis using propensity score matching. Dis Colon Rectum. 2017;60:266-273. [PubMed: 28177988]

107. Ghezzi TL, Luca F, Valvo M, et al. Robotic versus open total mesorectal excision for rectal cancer: comparative study of short and long-term outcomes. Eur J Surg Oncol. 2014;40:10721079. [PubMed: 24646748]

108. Kim JY, Kim NK, Lee KY, Hur H, Min BS, Kim JH. A comparative study of voiding and sexual function after total mesorectal excision with autonomic nerve preservation for rectal cancer: laparoscopic versus robotic surgery. Ann Surg Oncol. 2012;19:2485-2493. [PubMed: 22434245]

109. Luca F, Valvo M, Ghezzi TL, et al. Impact of robotic surgery on sexual and urinary functions after fully robotic nerve-sparing total mesorectal excision for rectal cancer. Ann Surg. 2013;257:672678. [PubMed: 23001075]

110. Sartori CA, Sartori A, Vigna S, Occhipinti R, Baiocchi GL. Urinary and sexual disorders after laparoscopic TME for rectal cancer in males. J Gastrointest Surg. 2011;15:637-643. [PubMed: 21327977]

111. Bosio R, Pigazzi A. Hybrid laparoscopic-robotic low anterior resection. In: Kim J, Garcia-Aguilar J, eds. Surgery for Cancers of the Gastrointestinal Tract: A Step-by-Step Approach. New York: Springer; 2015:247-262.

112. Hui VW, Guillem JG. Minimal access surgery for rectal cancer: an update. Nat Rev Gastroenterol Hepatol. 2014;11:158-165. [PubMed: 24192606]

113. Baik SH, Ko YT, Kang CM, et al. Robotic tumor-specific mesorectal excision of rectal cancer: short-term outcome of a pilot randomized trial. Surg Endosc. 2008;22:1601-1608. [PubMed: 18270772]

114. Baek JH, McKenzie S, Garcia-Aguilar J, Pigazzi A. Oncologic outcomes of robotic-assisted total mesorectal excision for the treatment of rectal cancer. Ann Surg. 2010;251:882-886. [PubMed: 20395863]

115. Pigazzi A, Luca F, Patriti A, et al. Multicentric study on robotic tumor-specific mesorectal excision for the treatment of rectal cancer. Ann Surg Oncol. 2010;17:1614-1620. [PubMed: 20087780] 
116. Bianchi PP, Ceriani C, Locatelli A, et al. Robotic versus laparoscopic total mesorectal excision for rectal cancer: a comparative analysis of oncological safety and short-term outcomes. Surg Endosc. 2010;24:2888-2894. [PubMed: 20526623]

117. Kwak JM, Kim SH, Kim J, Son DN, Baek SJ, Cho JS. Robotic vs laparoscopic resection of rectal cancer: short-term outcomes of a case-control study. Dis Colon Rectum. 2011;54:151-156. [PubMed: 21228661]

118. Patel CB, Ragupathi M, Ramos-Valadez DI, Haas EM. A three-arm (laparoscopic, hand-assisted, and robotic) matched-case analysis of intraoperative and postoperative outcomes in minimally invasive colorectal surgery. Dis Colon Rectum. 2011;54:144-150. [PubMed: 21228660]

119. Morelli L, Di Franco G, Guadagni S, et al. Robot-assisted total mesorectal excision for rectal cancer: case-matched comparison of short-term surgical and functional outcomes between the da Vinci Xi and Si. Surg Endosc. 2018;32:589-600. [PubMed: 28733738]

120. Teeple E, Bleday R. Open Technique for Low Anterior Resection In: Kim J, Garcia-Aguilar J, eds. Surgery for Cancers of the Gastrointestinal Tract: A Step-by-Step Approach. New York: Springer; 2015:215-226.

121. Rutkowski A, Bujko K, Nowacki MP, et al. Distal bowel surgical margin shorter than $1 \mathrm{~cm}$ after preoperative radiation for rectal cancer: is it safe? Ann Surg Oncol. 2008;15:3124-3131. [PubMed: 18766404]

122. Williams NS, Dixon MF, Johnston D. Reappraisal of the 5 centimetre rule of distal excision for carcinoma of the rectum: a study of distal intramural spread and of patients' survival. Br J Surg, 1983;70:150-154. [PubMed: 6831156]

123. Zureikat AH, Hogg ME, Zeh HJ, 3rd. The utility of the robot in pancreatic resections. Advances Surg, 2014;48:77-95. [PubMed: 25293609]

124. Zureikat AH, Borrebach J, Pitt HA, et al. Minimally invasive hepatopancreatobiliary surgery in North America: an ACS-NSQIP analysis of predictors of conversion for laparoscopic and robotic pancreatectomy and hepatectomy. HPB, 2017;19:595-602. [PubMed: 28400087]

125. Knab LM, Zureikat AH, Zeh HJ, 3rd, Hogg ME. Towards standardized robotic surgery in gastrointestinal oncology. Langenbeck's Arch Surgery, 2017;402:1003-1014.

126. Smith R, Patel V, Satava R. Fundamentals of robotic surgery: a course of basic robotic surgery skills based upon a 14-society consensus template of outcomes measures and curriculum development. Int J Med Robot. 2014;10:379-384. [PubMed: 24277315]

127. Hogg ME, Tam V, Zenati M, et al. Mastery-Based Virtual Reality Robotic Simulation Curriculum: The First Step Toward Operative Robotic Proficiency. J Surg Educ, 2017;74:477485. [PubMed: 27884677]

128. Tam V, Zenati M, Novak S, et al. Robotic pancreatoduodenectomy biotissue curriculum has validity and improves technical performance for surgical oncology fellows. J Surg Educ. 2017;74:1057-1065. [PubMed: 28578981]

129. Boone BA, Zenati M, Hogg ME, et al. Assessment of quality outcomes for robotic pancreaticoduodenectomy: identification of the learning curve. JAMA Surg. 2015;150:416-422. [PubMed: 25761143]

130. Shakir M, Boone BA, Polanco PM, et al. The learning curve for robotic distal pancreatectomy: an analysis of outcomes of the first 100 consecutive cases at a high-volume pancreatic centre. HPB. 2015;17:580-586. [PubMed: 25906690]

131. King JC, Zeh HJ, 3rd, Zureikat AH, et al. Safety in numbers: progressive implementation of a robotics program in an academic surgical oncology practice. Surg Innov. 2016;23:407-414 [PubMed: 27130645]

132. Napoli N, Kauffmann EF, Palmeri M, et al. The learning curve in robotic pancreaticoduodenectomy. Dig Surg. 2016;33:299-307. [PubMed: 27215422]

133. Napoli N, Kauffmann EF, Perrone VG, Miccoli M, Brozzetti S, Boggi U. The learning curve in robotic distal pancreatectomy. Updates Surgery. 2015;67:257-264.

134. Edil BH, Schulick RD. Challenges of minimally invasive pancreas surgery growth with such a high learning curve. JAMA Surg. 2015;150:423. [PubMed: 25760810] 
135. Hogg ME, Zenati M, Novak S, et al. Grading of surgeon technical performance predicts postoperative pancreatic fistula for pancreaticoduodenectomy independent of patient-related variables. Ann Surg. 2016;264:482-491. [PubMed: 27433897]

136. Schmidt CM, Turrini O, Parikh P, et al. Effect of hospital volume, surgeon experience, and surgeon volume on patient outcomes after pancreaticoduodenectomy: a single-institution experience. Arch Surg. 2010;145:634-640. [PubMed: 20644125]

137. Tseng JF, Pisters PW, Lee JE, et al. The learning curve in pancreatic surgery. Surgery. 2007;141:456-463. [PubMed: 17383522]

138. Memeo R, Sangiuolo F, de Blasi V, et al. Robotic pancreaticoduodenectomy and distal pancreatectomy: state of the art. Journal of visceral surgery, 2016;153:353-359. [PubMed: 27185566]

139. Zureikat AH, Postlewait LM, Liu Y, et al. A multi-institutional comparison of perioperative outcomes of robotic and open pancreaticoduodenectomy. Ann Surg. 2016;264:640-649. [PubMed: 27433907]

140. Chen S, Chen JZ, Zhan Q et al. Robot-assisted laparoscopic versus open pancreaticoduodenectomy: a prospective, matched, mid-term follow-up study. Surg Endosc. 2015;29:3698-3711. [PubMed: 25761559]

141. Lai EC, Yang GP, Tang CN. Robot-assisted laparoscopic pancreaticoduodenectomy versus open pancreaticoduodenectomy-a comparative study. Int J Surg. 2012;10:475-479. [PubMed: 22732431]

142. Buchs NC, Addeo P, Bianco FM, Ayloo S, Benedetti E, Giulianotti PC. Robotic versus open pancreaticoduodenectomy: a comparative study at a single institution. World J Surg. 2011;35:2739-2746. [PubMed: 21947494]

143. Chalikonda S, Aguilar-Saavedra JR, Walsh RM. Laparoscopic robotic-assisted pancreaticoduodenectomy: a case-matched comparison with open resection. Surg Endosc. 2012;26:2397-2402. [PubMed: 22437947]

144. McMillan MT, Zureikat AH, Hogg ME, et al. A propensity score-matched analysis of robotic vs open pancreatoduodenectomy on incidence of pancreatic fistula. JAMA Surg. 2017;152:327-335. [PubMed: 28030724]

145. Girgis MD, Zenati MS, Steve J, et al. Robotic approach mitigates perioperative morbidity in obese patients following pancreaticoduodenectomy. HPB. 2017;19:93-98. [PubMed: 28038966]

146. Magge D, Zureikat A, Hogg M, Zeh HJ, 3rd. Minimally invasive approaches to pancreatic surgery. Surg Oncol Clinics N Am. 2016;25:273-286.

147. Nguyen TK, Zenati MS, Boone BA, et al. Robotic pancreaticoduodenectomy in the presence of aberrant or anomalous hepatic arterial anatomy: safety and oncologic outcomes. HPB. 2015;17:594-599. [PubMed: 25913696]

148. Katz MH, Marsh R, Herman JM, et al. Borderline resectable pancreatic cancer: need for standardization and methods for optimal clinical trial design. Ann Surg Oncol. 2013;20:27872795. [PubMed: 23435609]

149. Boone BA, Bahary N, Zureikat AH, et al. Safety and biologic response of pre-operative autophagy inhibition in combination with gemcitabine in patients with pancreatic adenocarcinoma. Ann Surg Oncol. 2015;22:4402-4410. [PubMed: 25905586]

150. Boone BA, Steve J, Krasinskas AM, et al. Outcomes with FOLFIRINOX for borderline resectable and locally unresectable pancreatic cancer. J Surg Oncol. 2013;108:236-241. [PubMed: 23955427]

151. Boone BA, Steve J, Zenati MS, et al. Serum CA 19-9 response to neoadjuvant therapy is associated with outcome in pancreatic adenocarcinoma. Ann Surg Oncol. 2014;21:4351-4358. [PubMed: 25092157]

152. Faris JE, Blaszkowsky LS, McDermott S, et al. FOLFIRINOX in locally advanced pancreatic cancer: the Massachusetts General Hospital Cancer Center experience. The oncologist. 2013;18:543-548. [PubMed: 23657686]

153. Strobel O, Berens V, Hinz U, et al. Resection after neoadjuvant therapy for locally advanced, "unresectable" pancreatic cancer. Surgery. 2012;152:S33-S42. [PubMed: 22770956] 
154. Cunningham KE, Zenati MS, Petrie JR, et al. A policy of omitting an intensive care unit stay after robotic pancreaticoduodenectomy is safe and cost-effective. J Surg Res. 2016;204:8-14. [PubMed: 27451861]

155. Mack MJ. Minimally invasive and robotic surgery. Jama, 2001;285:568-572 [PubMed: 11176860]

156. Giulianotti PC, Sbrana F, Bianco FM, et al. Robot-assisted laparoscopic pancreatic surgery: single-surgeon experience. Surg Endosc, 2010;24:1646-1657 [PubMed: 20063016]

157. Butturini G, Damoli I, Crepaz L, et al. A prospective non-randomised single-center study comparing laparoscopic and robotic distal pancreatectomy. Surg Endosc, 2015;29:3163-3170. [PubMed: 25552231]

158. Lee SY, Allen PJ, Sadot E, et al. Distal pancreatectomy: a single institution's experience in open, laparoscopic, and robotic approaches. J Am Coll Surg, 2015;220:18-27 [PubMed: 25456783]

159. Lai EC, Tang CN. Robotic distal pancreatectomy versus conventional laparoscopic distal pancreatectomy: a comparative study for short-term outcomes. Front Med, 2015;9:356-360. [PubMed: 26271291]

160. Chen S, Zhan Q Chen JZ, et al. Robotic approach improves spleen-preserving rate and shortens postoperative hospital stay of laparoscopic distal pancreatectomy: a matched cohort study. Surg Endosc. 2015;29:3507-3518. [PubMed: 25791063]

161. Zureikat AH, Nguyen KT, Bartlett DL, Zeh HJ, Moser AJ. Robotic-assisted major pancreatic resection and reconstruction. Arch Surg, 2011;146:256-261 [PubMed: 21079111]

162. Daouadi M, Zureikat AH, Zenati MS, et al. Robot-assisted minimally invasive distal pancreatectomy is superior to the laparoscopic technique. Ann Surg. 2013;257:128-132 [PubMed: 22868357]

163. Wu X, Tao R, Lei R, et al. Distal pancreatectomy combined with celiac axis resection in treatment of carcinoma of the body/tail of the pancreas: a single-center experience. Ann Surg Oncol, 2010;17:1359-1366 [PubMed: 20198445]

164. Baumgartner JM, Krasinskas A, Daouadi M, et al. Distal pancreatectomy with en bloc celiac axis resection for locally advanced pancreatic adenocarcinoma following neoadjuvant therapy. $\mathrm{J}$ Gastrointest Surg. 2012;16:1152-1159. [PubMed: 22399269]

165. Hirano S, Kondo S, Hara T, et al. Distal pancreatectomy with en bloc celiac axis resection for locally advanced pancreatic body cancer: long-term results. Ann Surg. 2007;246:46-51. [PubMed: 17592290]

166. Ocuin LM, Miller-Ocuin JL, Novak SM, et al. Robotic and open distal pancreatectomy with celiac axis resection for locally advanced pancreatic body tumors: a single institutional assessment of perioperative outcomes and survival. HPB. 2016;18:835-842. [PubMed: 27506992]

167. Iacono C, Ruzzenente A, Bortolasi L, Guglielmi A. Central pancreatectomy: the Dagradi Serio Iacono operation. Evolution of a surgical technique from the pioneers to the robotic approach. World J Gastroenterol. 2014;20:15674-15681. [PubMed: 25400451]

168. Goudard Y, Gaujoux S, Dokmak S, et al. Reappraisal of central pancreatectomy a 12-year singlecenter experience. JAMA Surg. 2014;149:356-363. [PubMed: 24740703]

169. Kang CM, Kim DH, Lee WJ, Chi HS. Initial experiences using robot-assisted central pancreatectomy with pancreaticogastrostomy: a potential way to advanced laparoscopic pancreatectomy. Surg Endosc. 2011;25:1101-1106. [PubMed: 20835724]

170. Zureikat AH, Moser AJ, Boone BA, Bartlett DL, Zenati M, Zeh HJ, 3rd. 250 robotic pancreatic resections: safety and feasibility. Ann Surg. 2013;258:554-559 discussion 9-62. [PubMed: 24002300]

171. Chen S, Zhan Q, Jin JB, et al. Robot-assisted laparoscopic versus open middle pancreatectomy: short-term results of a randomized controlled trial. Surg Endosc, 2017;31:962-971. [PubMed: 27402095]

172. Hamad A, Novak S, Hogg ME. Robotic central pancreatectomy. J Vis Surgery. 2017;3:94,

173. Abood GJ, Can MF, Daouadi M, et al. Robotic-assisted minimally invasive central pancreatectomy: technique and outcomes. J Gastrointest Surg. 2013;17:1002-1008. [PubMed: 23325340] 
174. Heidt DG, Burant C, Simeone DM. Total pancreatectomy: indications, operative technique, and postoperative sequelae. J Gastrointest Surg. 2007;11:209-216. [PubMed: 17390175]

175. Galvani CA, Rodriguez Rilo H, Samame J, Porubsky M, Rana A, Gruessner RW. Fully roboticassisted technique for total pancreatectomy with an autologous islet transplant in chronic pancreatitis patients: results of a first series. J Am Coll Surg. 2014;218:e73-e78. [PubMed: 24559970]

176. Zureikat AH, Nguyen T, Boone BA, et al. Robotic total pancreatectomy with or without autologous islet cell transplantation: replication of an open technique through a minimal access approach. Surg Endosc. 2015;29:176-183. [PubMed: 25005012]

177. Baron TH, Harewood GC, Morgan DE, Yates MR. Outcome differences after endoscopic drainage of pancreatic necrosis, acute pancreatic pseudocysts, and chronic pancreatic pseudocysts. Gastrointest Endosc, 2002;56:7-17. [PubMed: 12085029]

178. Stafford AT, Walsh RM. Robotic surgery of the pancreas: the current state of the art. J Surg Oncol. 2015;112:289-294. [PubMed: 26220683]

179. Nassour I, Ramzan Z, Kukreja S. Robotic cystogastrostomy and debridement of walled-off pancreatic necrosis. J Robot Surg. 2016;10:279-282. [PubMed: 27039191]

180. Khreiss M, Zenati M, Clifford A, et al. Cyst gastrostomy and necrosectomy for the management of sterile walled-off pancreatic necrosis: a comparison of minimally invasive surgical and endoscopic outcomes at a high-volume pancreatic center. J Gastrointest Surg. 2015;19:14411448. [PubMed: 26033038]

181. Siegel R, Ma J, Zou Z, Jemal A. Cancer statistics, 2014. CA Cancer J Clin, 2014;64:9-29. [PubMed: 24399786]

182. Blot WJ, Devesa SS, Kneller RW, Fraumeni JF, Jr. Rising incidence of adenocarcinoma of the esophagus and gastric cardia. JAMA. 1991;265:1287-1289. [PubMed: 1995976]

183. Hansson LE, Sparen P, Nyren O. Increasing incidence of carcinoma of the gastric cardia in Sweden from 1970 to 1985. Br J Surg. 1993;80:374-377. [PubMed: 8472157]

184. Anderson WF, Camargo MC, Fraumeni JF, Jr, Correa P, Rosenberg PS, Rabkin CS. Age-specific trends in incidence of noncardia gastric cancer in US adults. JAMA. 2010;303:1723-1728. [PubMed: 20442388]

185. Herrera-Almario G, Strong VE. Minimally invasive gastric surgery. Ann Surg Oncol. 2016;23:3792-3797 [PubMed: 27489058]

186. Theuer CP. Asian gastric cancer patients at a southern California comprehensive cancer center are diagnosed with less advanced disease and have superior stage-stratified survival. Am Surg. 2000;66:821-826. [PubMed: 10993608]

187. Theuer CP, Kurosaki T, Ziogas A, Butler J, Anton-Culver H. Asian patients with gastric carcinoma in the United States exhibit unique clinical features and superior overall and cancer specific survival rates. Cancer. 2000;89:1883-1892. [PubMed: 11064344]

188. Strong VE, Song KY, Park CH, et al. Comparison of gastric cancer survival following R0 resection in the United States and Korea using an internationally validated nomogram. Ann Surg. 2010;251:640-646. [PubMed: 20224369]

189. Schwarz RE, Zagala-Nevarez K. Ethnic survival differences after gastrectomy for gastric cancer are better explained by factors specific for disease location and individual patient comorbidity. Eur J Surg Oncol. 2002;28:214-219. [PubMed: 11944952]

190. Yao JC, Schnirer II, Reddy S, et al. Effects of sex and racial/ethnic group on the pattern of gastric cancer localization. Gastric Cancer. 2002;5:208-212. [PubMed: 12491078]

191. Verdecchia A, Mariotto A, Gatta G, Bustamante-Teixeira MT, Ajiki W. Comparison of stomach cancer incidence and survival in four continents. Eur J Cancer. 2003;39:1603-1609. [PubMed: 12855268]

192. Vinuela EF, Gonen M, Brennan MF, Coit DG, Strong VE. Laparoscopic versus open distal gastrectomy for gastric cancer: a meta-analysis of randomized controlled trials and high-quality nonrandomized studies. Ann Surg. 2012;255:446-456 [PubMed: 22330034]

193. Kelly KJ, Selby L, Chou JF, et al. Laparoscopic versus open gastrectomy for gastric adenocarcinoma in the west: a case-control study. Ann Surg Oncol. 2015;22:3590-3596 [PubMed: 25631063] 
194. Hashizume M, Sugimachi K. Robot-assisted gastric surgery. Surg Clinics N Am. 2003;83:14291444

195. Giulianotti PC, Coratti A, Angelini M, et al. Robotics in general surgery: personal experience in a large community hospital. Arch Surg. 2003;138:777-784 [PubMed: 12860761]

196. Anderson C, Ellenhorn J, Hellan M, Pigazzi A. Pilot series of robot-assisted laparoscopic subtotal gastrectomy with extended lymphadenectomy for gastric cancer. Surg Endosc. 2007;21:16621666 [PubMed: 17345142]

197. Hyun MH, Lee CH, Kwon YJ, et al. Robot versus laparoscopic gastrectomy for cancer by an experienced surgeon: comparisons of surgery, complications, and surgical stress. Ann Surg Oncol. 2013;20:1258-1265. [PubMed: 23080320]

198. Kang BH, Xuan Y, Hur H, Ahn CW, Cho YK, Han SU. Comparison of surgical outcomes between robotic and laparoscopic gastrectomy for gastric cancer: the learning curve of robotic surgery. J Gastric Cancer. 2012;12:156-163 [PubMed: 23094227]

199. Woo Y, Hyung WJ, Pak KH, et al. Robotic gastrectomy as an oncologically sound alternative to laparoscopic resections for the treatment of early-stage gastric cancers. Arch Surg. 2011;146:1086-1092. [PubMed: 21576595]

200. Song J, Oh SJ, Kang WH, Hyung WJ, Choi SH, Noh SH. Robot-assisted gastrectomy with lymph node dissection for gastric cancer: lessons learned from an initial 100 consecutive procedures. Ann Surg. 2009;249:927-932. [PubMed: 19474671]

201. Kim HI, Park MS, Song KJ, Woo Y, Hyung WJ. Rapid and safe learning of robotic gastrectomy for gastric cancer: multidimensional analysis in a comparison with laparoscopic gastrectomy. Eur J Surg Oncol. 2014;40:1346-1354 [PubMed: 24080199]

202. Kim KM, An JY, Kim HI, Cheong JH, Hyung WJ, Noh SH. Major early complications following open, laparoscopic and robotic gastrectomy. Br J Surg. 2012;99:1681-1687. [PubMed: 23034831]

203. Kim MC, Heo GU, Jung GJ. Robotic gastrectomy for gastric cancer: surgical techniques and clinical merits. Surg Endosc. 2010;24:610-615. [PubMed: 19688399]

204. Strong VE, Gholami S, Shah MA, et al. Total gastrectomy for hereditary diffuse gastric cancer at a single center: postsurgical outcomes in 41 patients. Ann Surg. 2017;266:1006-1012 [PubMed: 27759617]

205. Park JY, Jo MJ, Nam BH, et al. Surgical stress after robot-assisted distal gastrectomy and its economic implications. Br J Surg. 2012;99:1554-1561. [PubMed: 23027072]

206. Song J, Kang WH, Oh SJ, Hyung WJ, Choi SH, Noh SH. Role of robotic gastrectomy using da Vinci system compared with laparoscopic gastrectomy: initial experience of 20 consecutive cases. Surg Endosc. 2009;23:1204-1211. [PubMed: 19263147]

207. Park SS, Kim MC, Park MS, Hyung WJ. Rapid adaptation of robotic gastrectomy for gastric cancer by experienced laparoscopic surgeons. Surg Endosc. 2012;26:60-67. [PubMed: 21789643]

208. Coratti A, Annecchiarico M, Di Marino M, Gentile E, Coratti F, Giulianotti PC. Robot-assisted gastrectomy for gastric cancer: current status and technical considerations. World J Surg. 2013;37:2771-2781. [PubMed: 23674257]

209. LaFemina J, Vinuela EF, Schattner MA, Gerdes H, Strong VE. Esophagojejunal reconstruction after total gastrectomy for gastric cancer using a transorally inserted anvil delivery system. Ann Surg Oncol. 2013;20:2975-2983. [PubMed: 23584558]

210. Kelly KJ, Allen PJ, Brennan MF, Gollub MJ, Coit DG, Strong VE. Internal hernia after gastrectomy for cancer with Roux-Y reconstruction. Surgery. 2013;154:305-311. [PubMed: 23889956]

211. Patriti A, Ceccarelli G, Bellochi R, et al. Robot-assisted laparoscopic total and partial gastric resection with D2 lymph node dissection for adenocarcinoma. Surg Endosc. 2008;22:2753-2760. [PubMed: 18813994]

212. Isogaki J, Haruta S, Man IM, et al. Robot-assisted surgery for gastric cancer: experience at our institute. Pathobiology. 2011;78:328-333. [PubMed: 22104204] 
213. D’ Annibale A, Pende V, Pernazza G, et al. Full robotic gastrectomy with extended (D2) lymphadenectomy for gastric cancer: surgical technique and preliminary results. J Surg Res. 2011;166:e113-e120. [PubMed: 21227455]

214. Lee HH, Hur H, Jung H, Jeon HM, Park CH, Song KY. Robot-assisted distal gastrectomy for gastric cancer: initial experience. Am J Surg. 2011;201:841-845. [PubMed: 21741513]

215. Park JY, Kim YW, Ryu KW, Eom BW, Yoon HM, Reim D. Emerging role of robot-assisted gastrectomy: analysis of consecutive 200 cases. J Gastric Cancer. 2013;13:255-262. [PubMed: 24511422]

216. Tokunaga M, Sugisawa N, Kondo J, et al. Early phase II study of robot-assisted distal gastrectomy with nodal dissection for clinical stage IA gastric cancer. Gastric Cancer. 2014;17:542-547. [PubMed: 24005955]

217. Marano A, Choi YY, Hyung WJ, Kim YM, Kim J, Noh SH. Robotic versus laparoscopic versus open gastrectomy: a meta-analysis. J Gastric Cancer. 2013;13:136-148. [PubMed: 24156033]

218. Liao GX, Xie GZ, Li R, et al. Meta-analysis of outcomes compared between robotic and laparoscopic gastrectomy for gastric cancer. Asian Pac J Cancer Prev. 2013;14:4871-4875. [PubMed: 24083761]

219. Xiong B, Ma L, Zhang C. Robotic versus laparoscopic gastrectomy for gastric cancer: a metaanalysis of short outcomes. Surg Oncol. 2012;21:274-280. [PubMed: 22789391]

220. Xiong J, Nunes QM, Tan C, et al. Comparison of short-term clinical outcomes between robotic and laparoscopic gastrectomy for gastric cancer: a meta-analysis of 2495 patients. J Laparoendosc Adv Surg Tech A. 2013;23:965-976. [PubMed: 24093968]

221. Nakauchi M, Suda K, Susumu S, et al. Comparison of the long-term outcomes of robotic radical gastrectomy for gastric cancer and conventional laparoscopic approach: a single institutional retrospective cohort study. Surg Endosc. 2016;30:5444-5452. [PubMed: 27129542] 


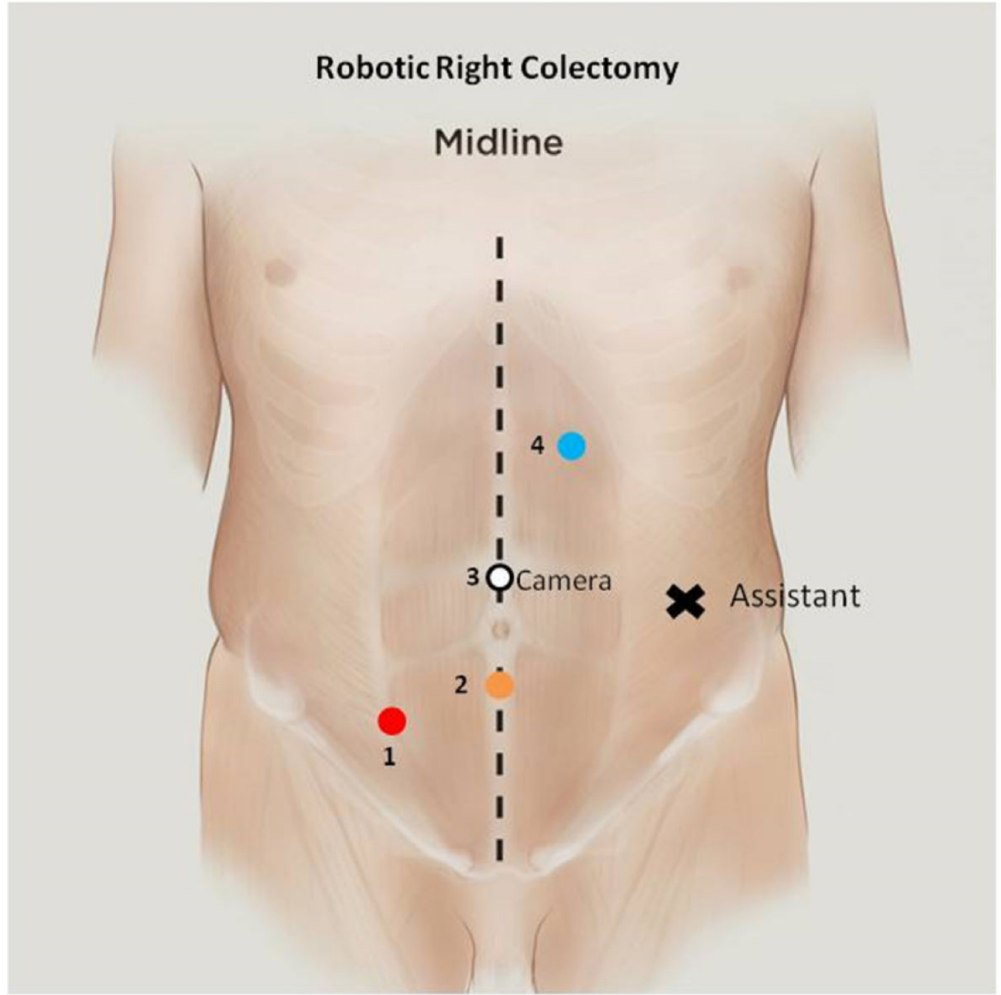

Fig. 1.

Robotic right colectomy port positions. 


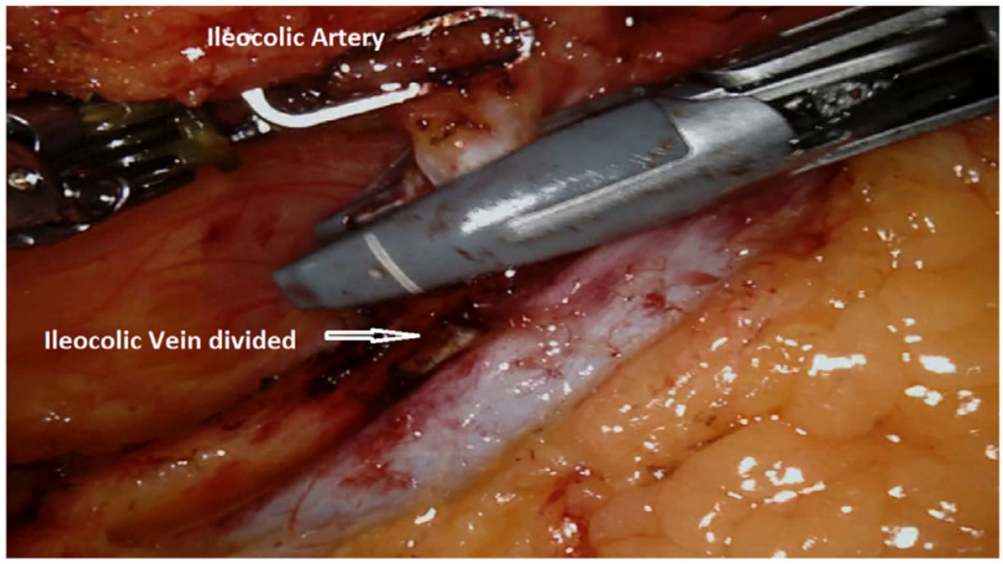

Fig. 2.

Ileocolic artery division. The ileocolic vein has been previously divided using the vessel sealer. 


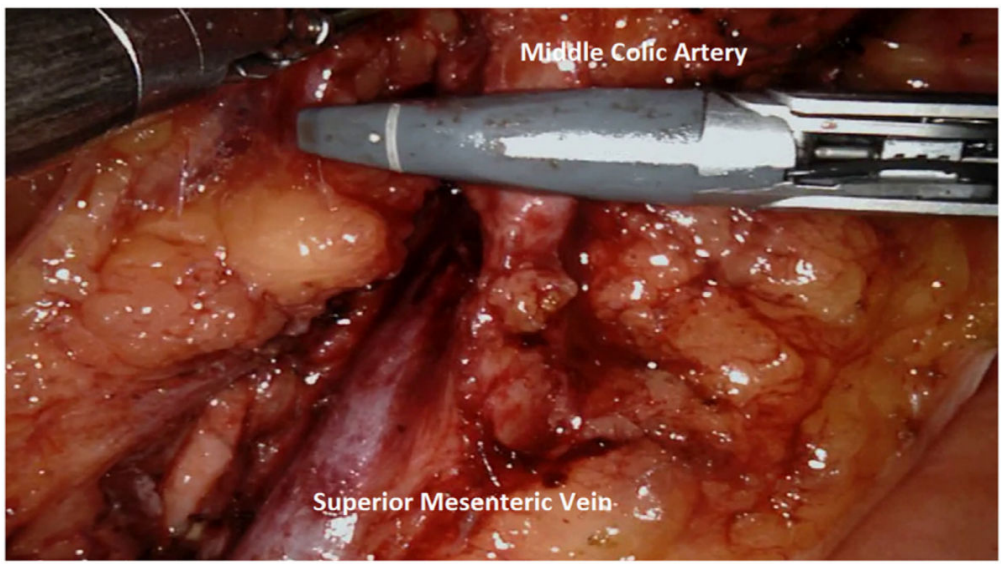

Fig. 3.

Division of the middle colic artery using a vessel sealer. 


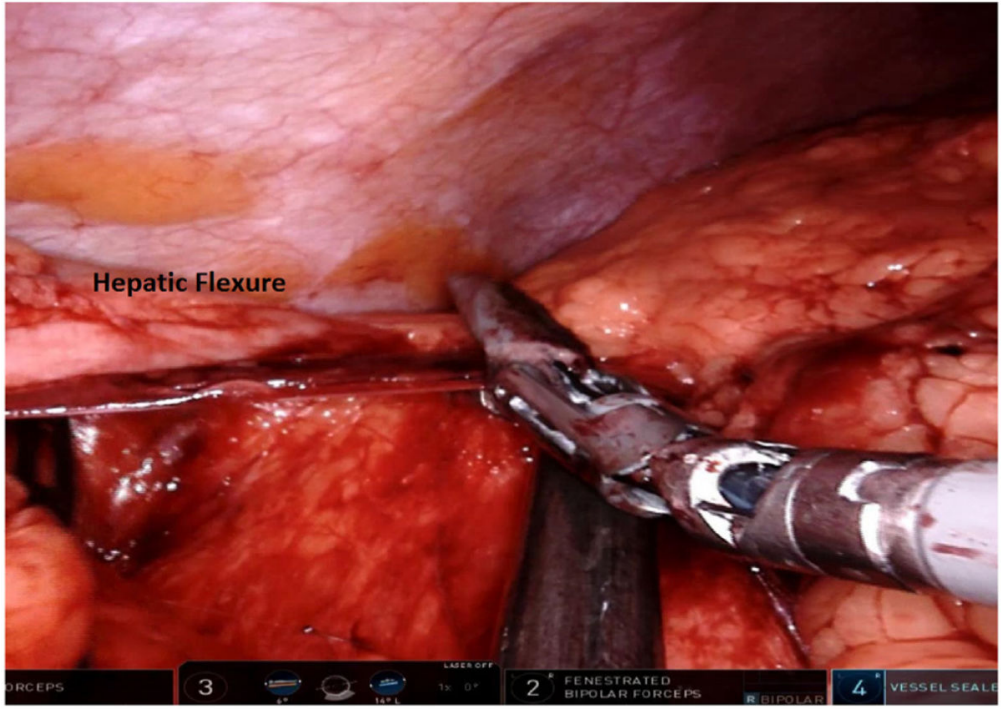

Fig. 4.

Dissection of the hepatic flexure. 


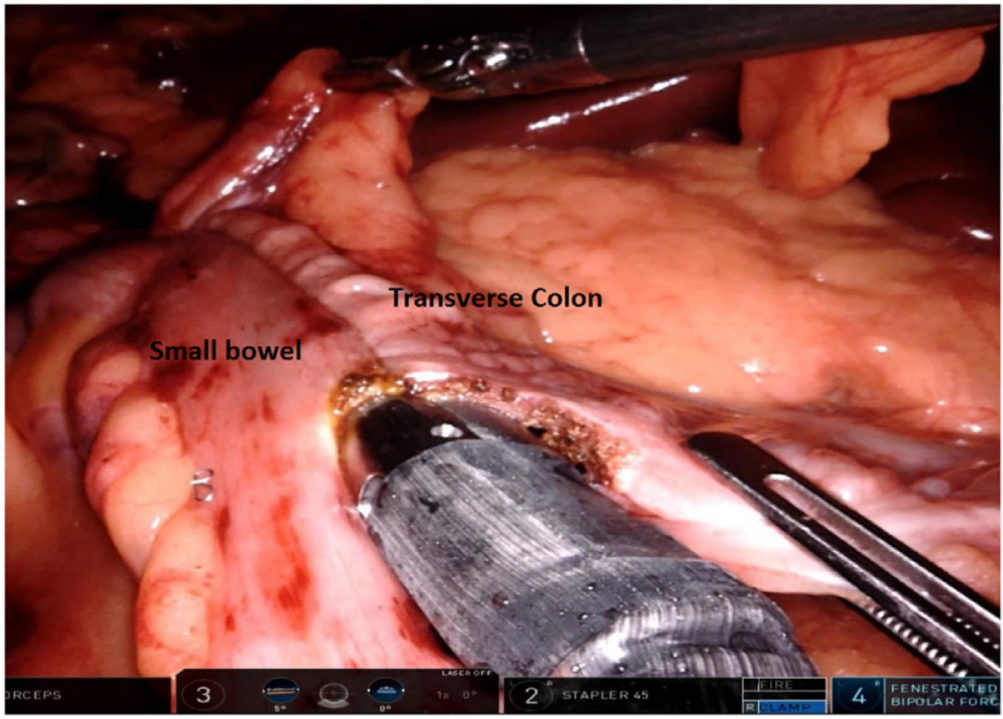

Fig. 5.

Intracorporeal isoperistaltic anastomosis between the terminal ileum and transverse colon. 


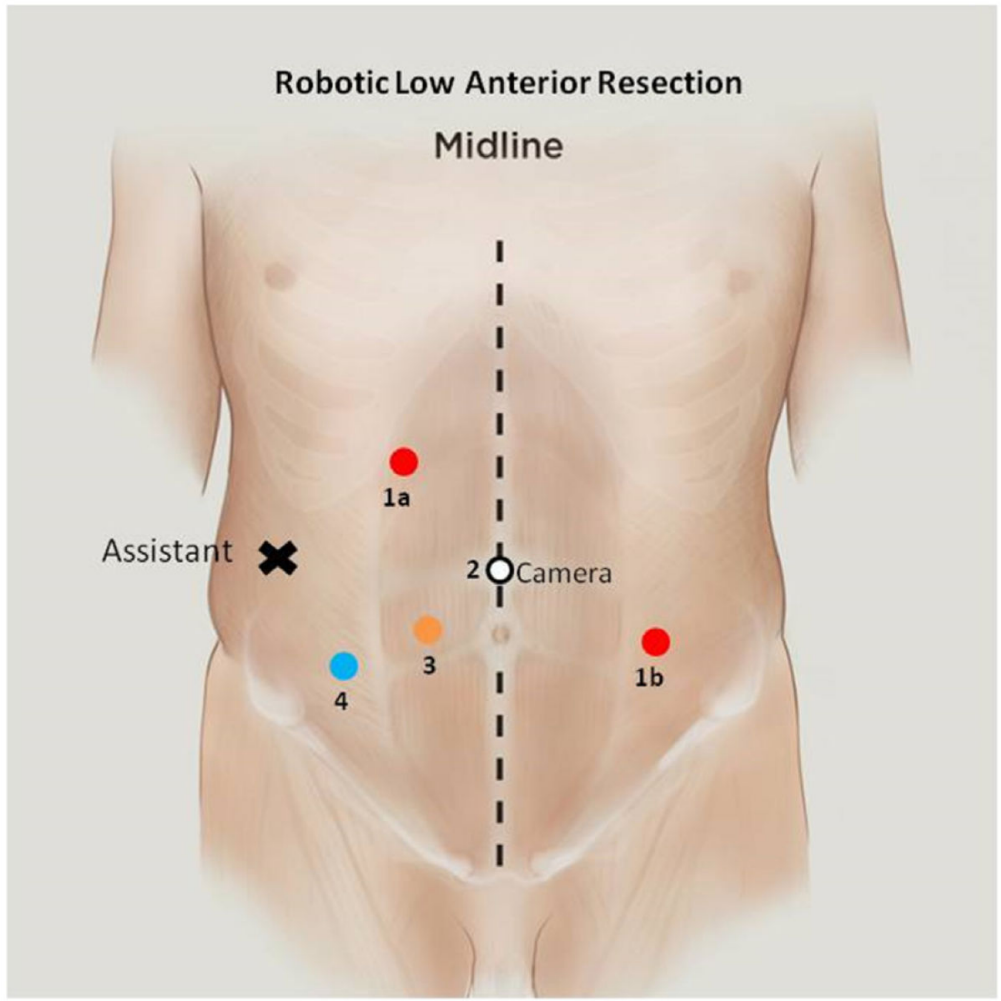

Fig. 6.

Port placement for robotic low anterior resection. For pedicle ligation and splenic flexure, mobilization ports 1a, 2, and 3 are used. For pelvic dissection, ports 1b, 2, and 3 are used. 


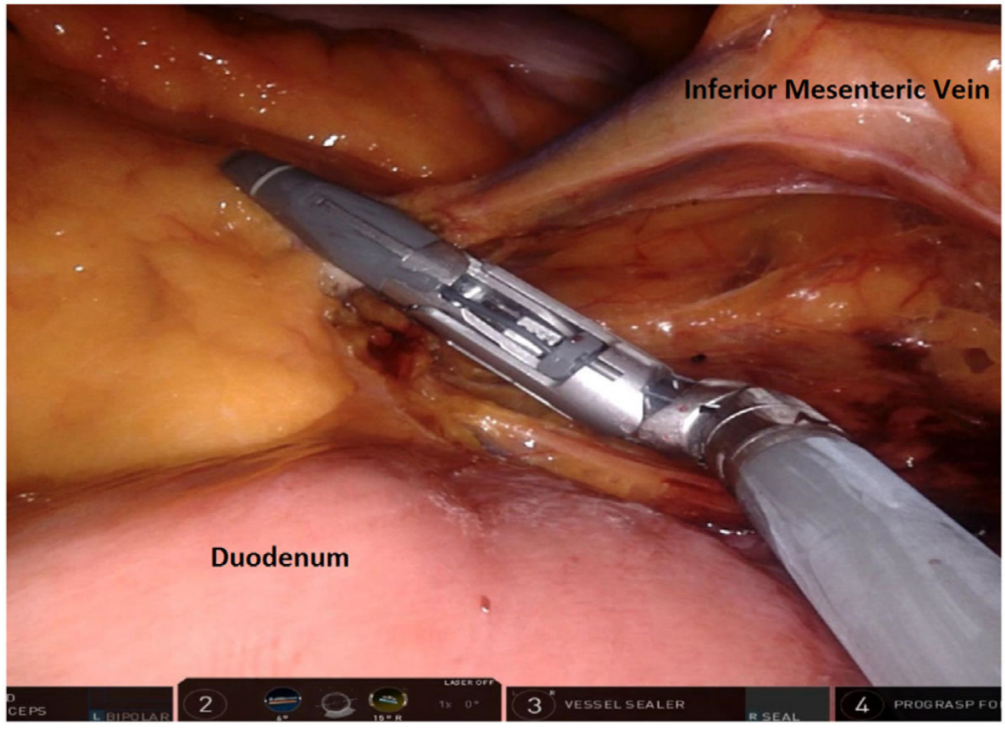

Fig. 7.

Inferior mesenteric vein dissected adjacent to the ligament of Treitz and inferior border of the pancreas before division. 


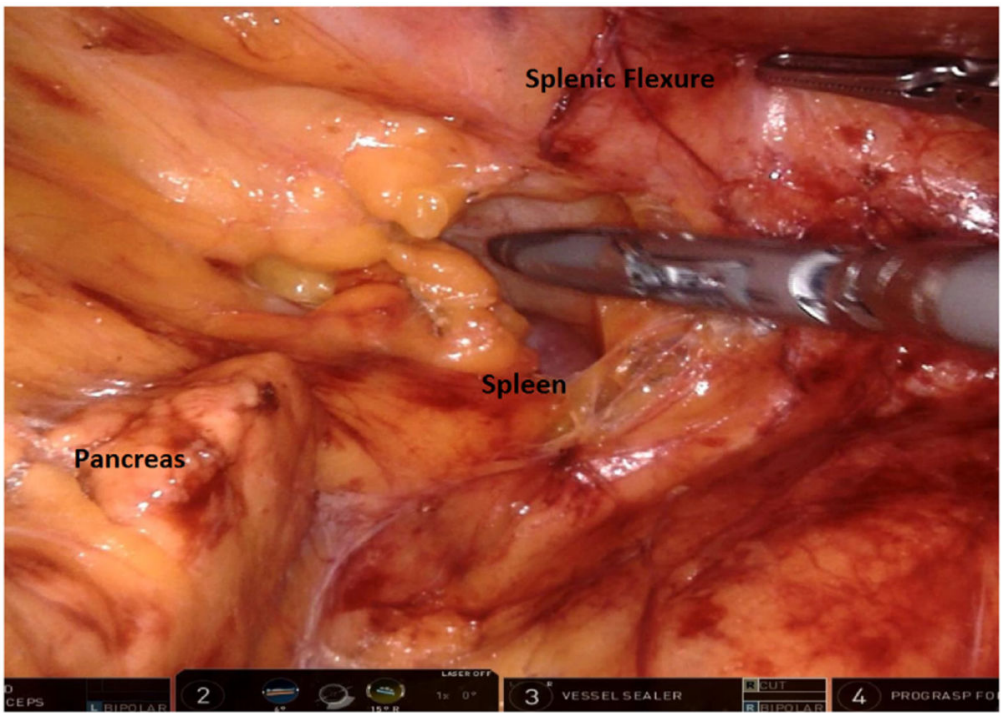

Fig. 8.

Medial-to-lateral dissection revealing the pancreas, prior to visualization of the spleen. The splenic flexure of the colon is gently retracted using the grasper. 


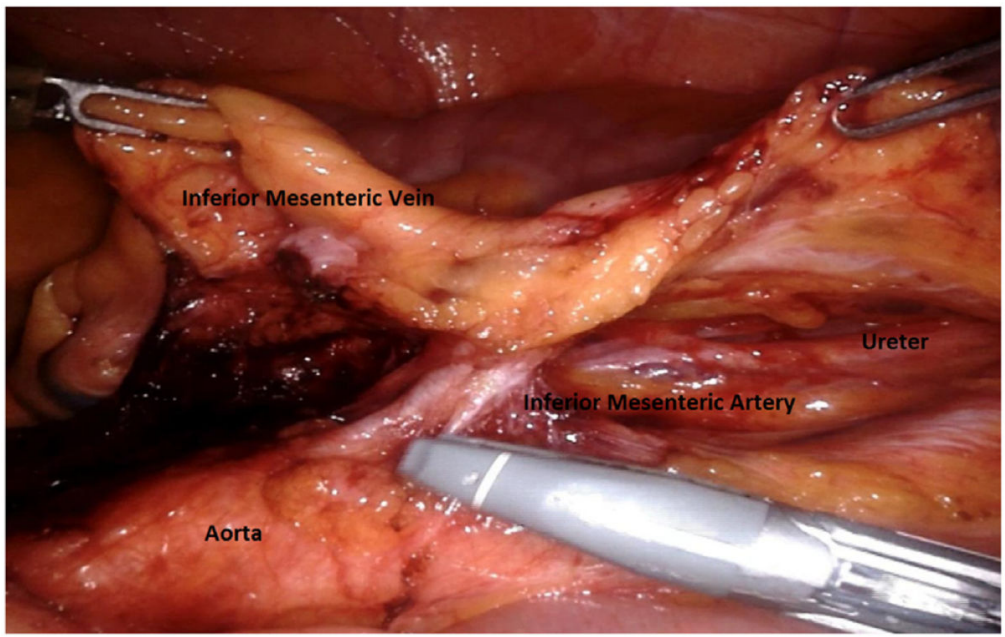

Fig. 9.

Inferior mesenteric artery (note characteristic T-shaped structure) after dividing the inferior mesenteric vein. The ureter and gonadal vessels are visible prior to artery division. 


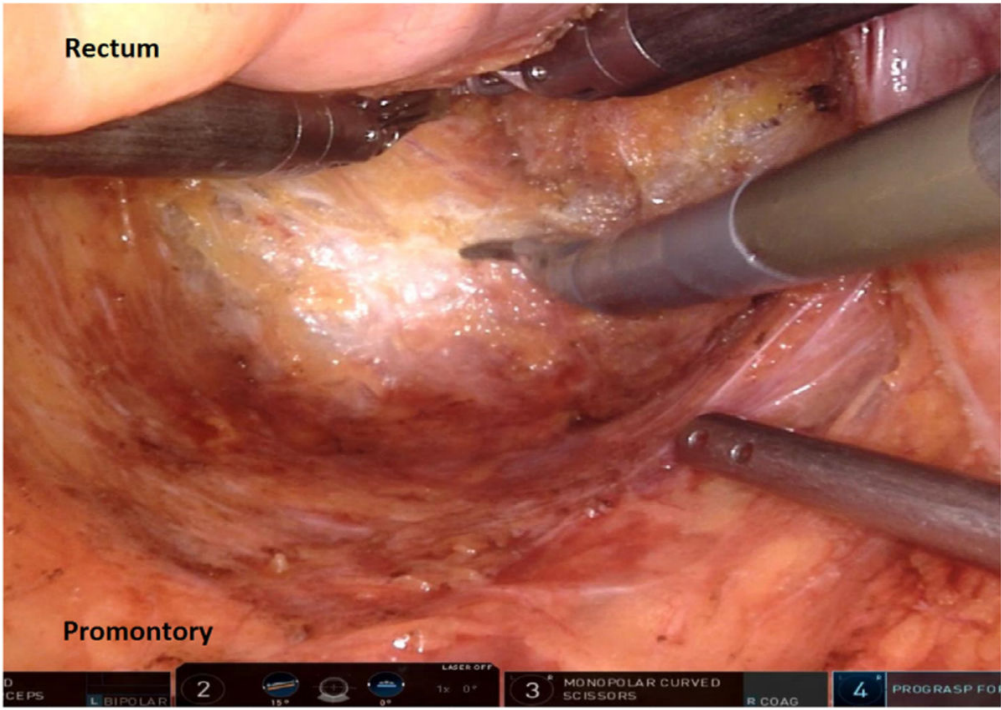

Fig. 10.

Posterior mesorectal excision plane. The hypogastric nerves are swept lateral during dissection. 


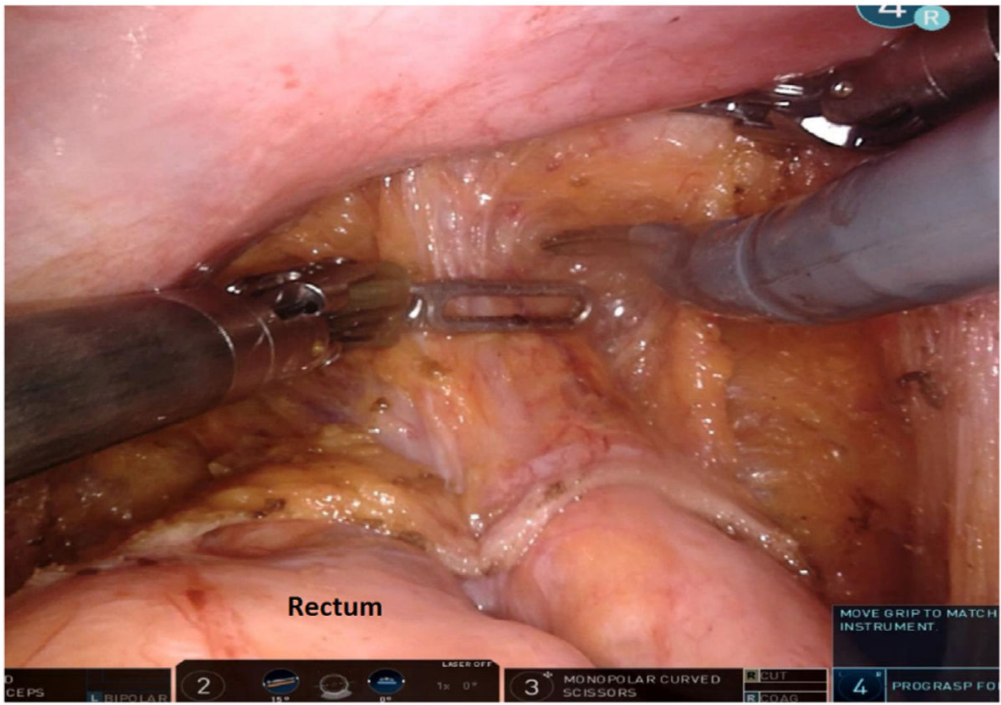

Fig. 11.

After the peritoneal plane is incised, dissection should continue along the rectovaginal septum in women or rectovesical fascia in men. 


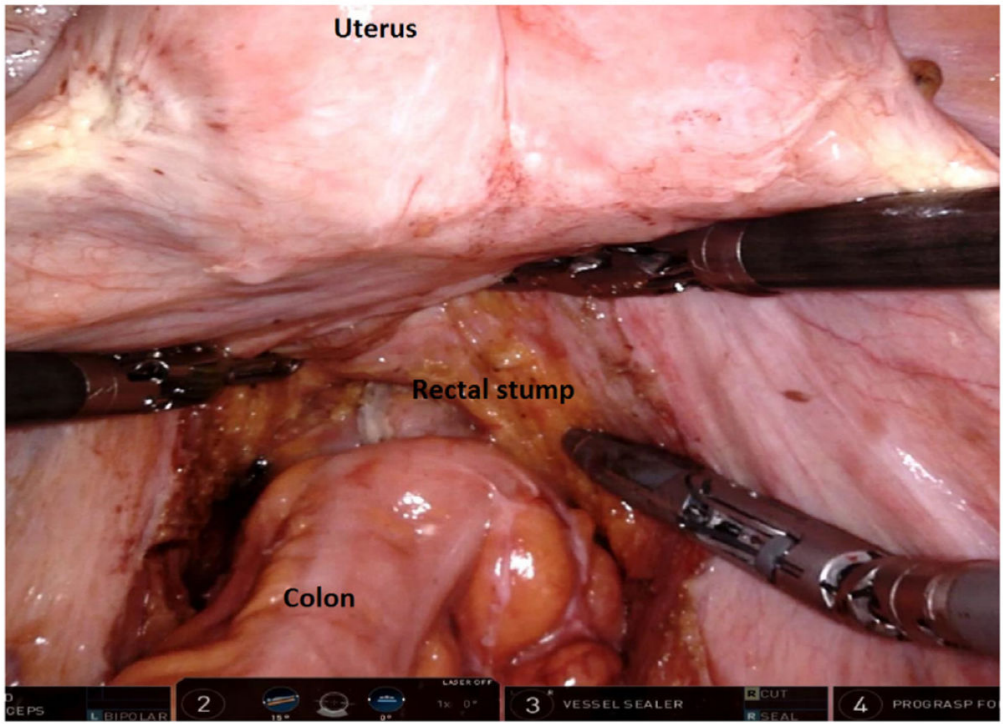

Fig. 12.

End-to-end intracorporeal stapled anastomosis between the descending colon and the rectal stump. The pelvic space is completely exposed, and the uterus can be retracted using a stitch. 
(a)

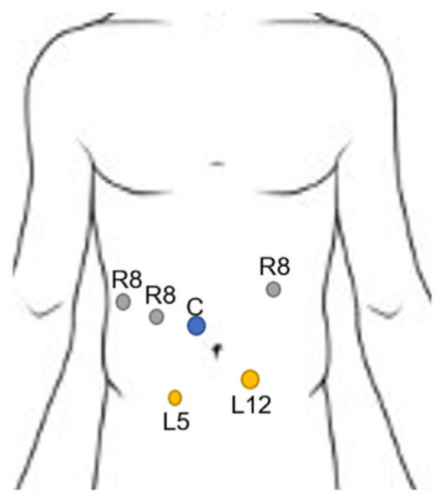

(b)

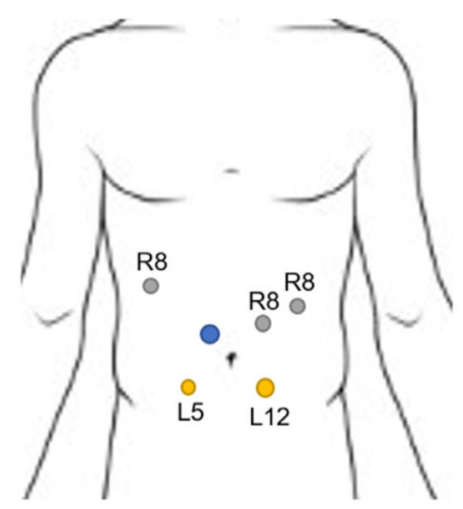

Fig. 13.

Port placements for (a) robotic pancreaticoduodenectomy, and (b) robotic distal pancreatectomy. C: Camera port, $\mathrm{R} 8: 8 \mathrm{~mm}$ robotic trocar, L5: $5 \mathrm{~mm}$ laparoscopic trocar, L12: $12 \mathrm{~mm}$ laparoscopic trocar. 

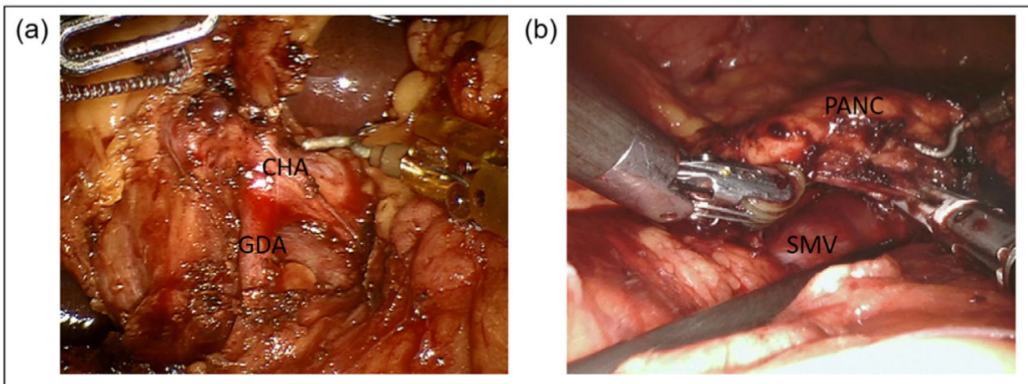

(c)

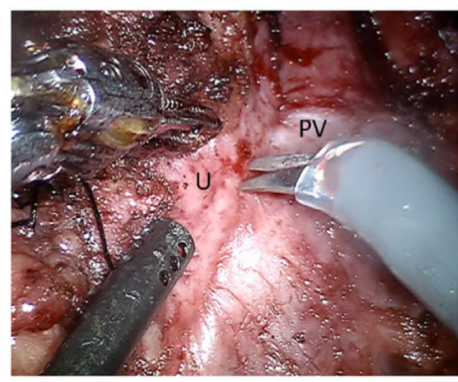

(d)

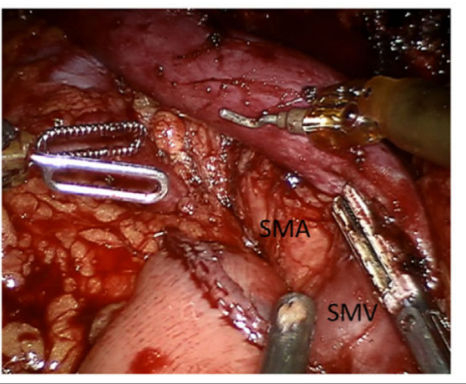

Fig. 14.

Robotic pancreaticoduodenectomy dissection and resection. (a) Porta hepatis dissection and identification of the gastroduodenal artery during robotic pancreaticoduodenectomy. (b) Creation of retropancreatic tunnel anterior to the superior mesenteric vein along the inferior border of the pancreatic neck. (c) Uncinate process dissection. (d) Completed dissection demonstrating exposed superior mesenteric vein, superior mesenteric artery and proximal jejunum. CHA, common hepatic artery; GDA, gastroduodenal artery; PANC, pancreas; PV, portal vein; SMA, superior mesenteric artery; SMV, superior mesenteric vein; U, uncinate. 


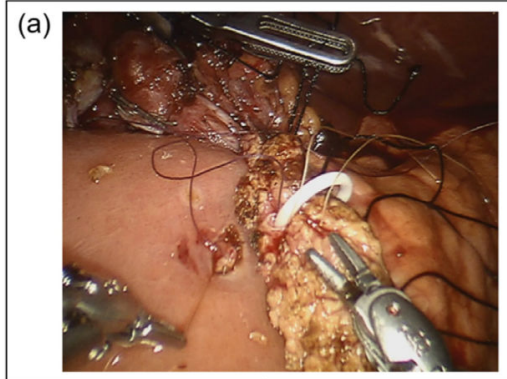

(b)

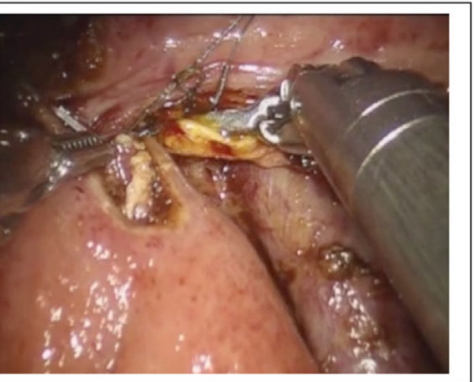

Fig. 15.

Robotic pancreaticoduodenectomy reconstruction. (a) Modified Blumgart pancreaticojejunostomy with pancre atic stent in place. (b) Continuous running hepaticojejunostomy. 
(a)

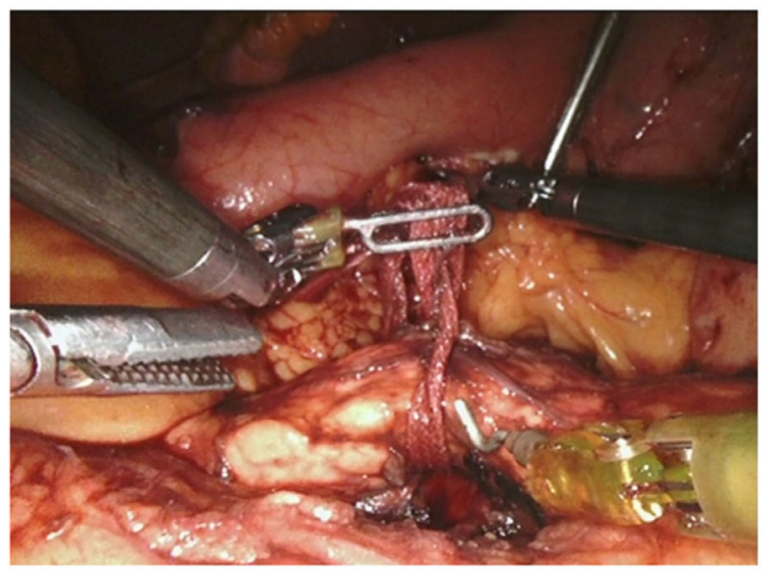

(b)

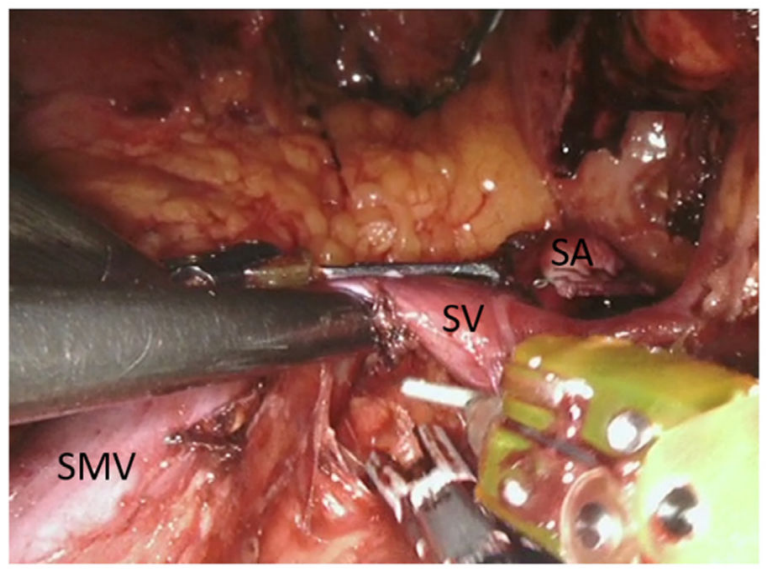

(c)

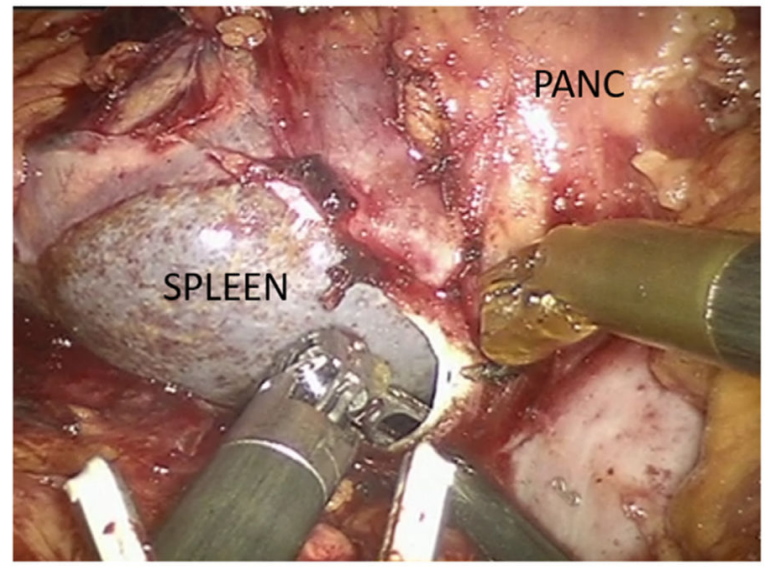

Fig. 16.

Robotic distal pancreatectomy dissection. (a) Creation of retropancreatic tunnel and passage of moist umbilical tape for upwards traction. (b) Division of the splenic artery at its takeoff and identification of the splenic vein for concurrent splenectomy. (c) Medial to lateral dissection of the pancreas and posterior pancreatic fascia. PANC, pancreas; SA, splenic artery; SMV, superior mesenteric vein; SV, splenic vein. 


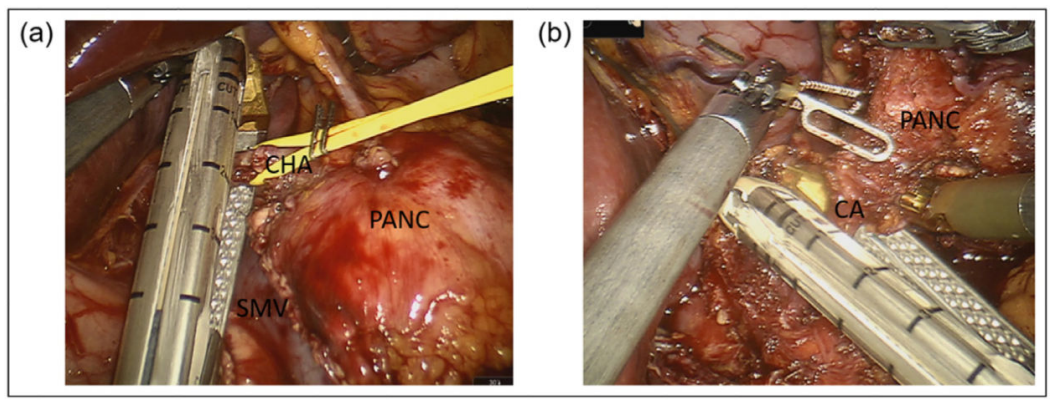

Fig. 17.

Robotic distal pancreatectomy with celiac axis resection. (a) Dissection and division of the common hepatic artery, ensuring protection of the gastroduodenal artery. (b) Transection of the celiac axis after circumferential dissection and lymphadenectomy. CA, celiac axis; CHA, common hepatic artery; PANC, pancreas; SMV, superior mesenteric vein. 


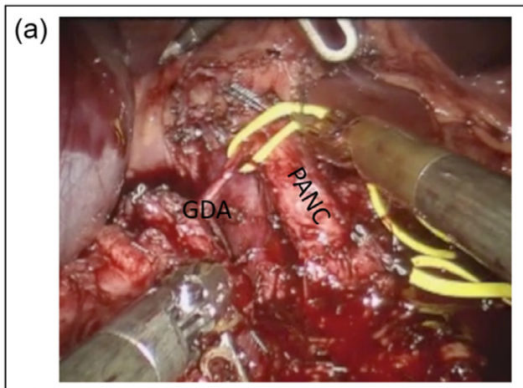

(b)

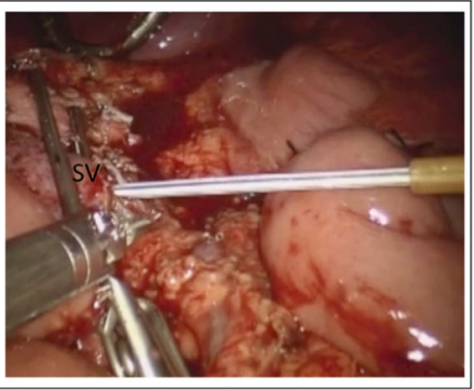

Fig. 18.

Robotic total pancreatectomy with islet autotransplantation. (a) Dissection of the whole pancreas without division at the neck. Gastroduodenal artery, inferior mesenteric vein, and splenic vessels preserved until dissection is complete. (b) Splenic vein cannulation for infusion of autoislets. GDA, gastroduodenal artery; PANC, pancreas. 


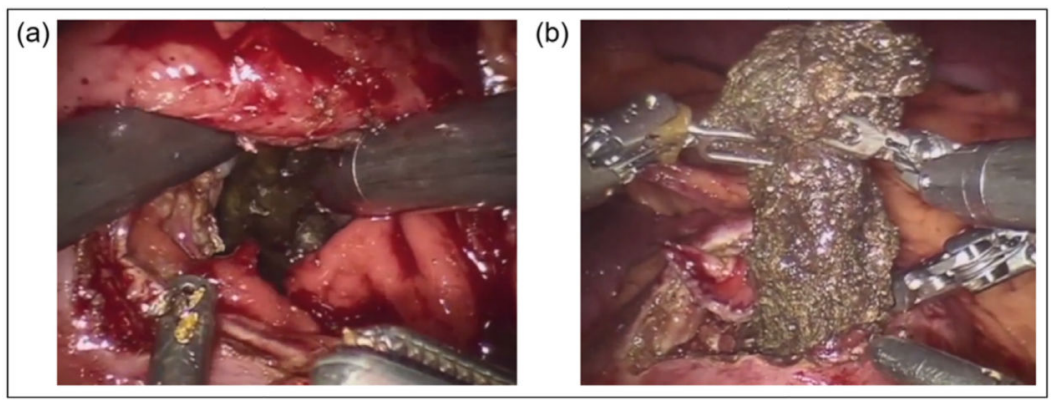

Fig. 19.

Robotic cyst gastrostomy. (a) Cyst gastrostomy performed with anterior and posterior gastrostomy into walled off necrosis of the pancreas. (b) Debridement of pancreatic necrosis. 


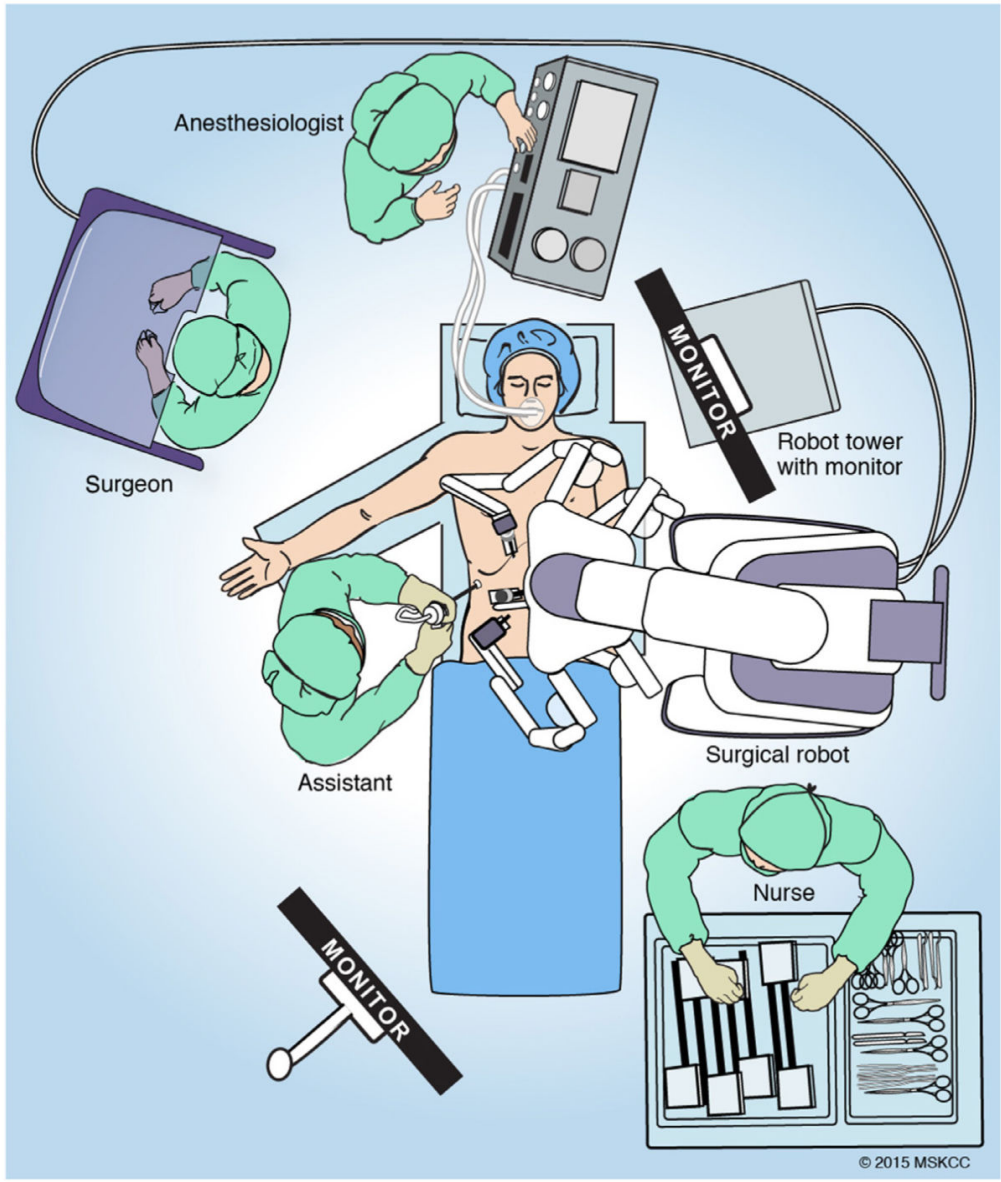

Fig. 20.

Position for a patient undergoing robotic total gastrectomy. Of note, the operation is generally performed in steep reverse Trendelenburg position and the patient must be positioned prior to docking the robot. 


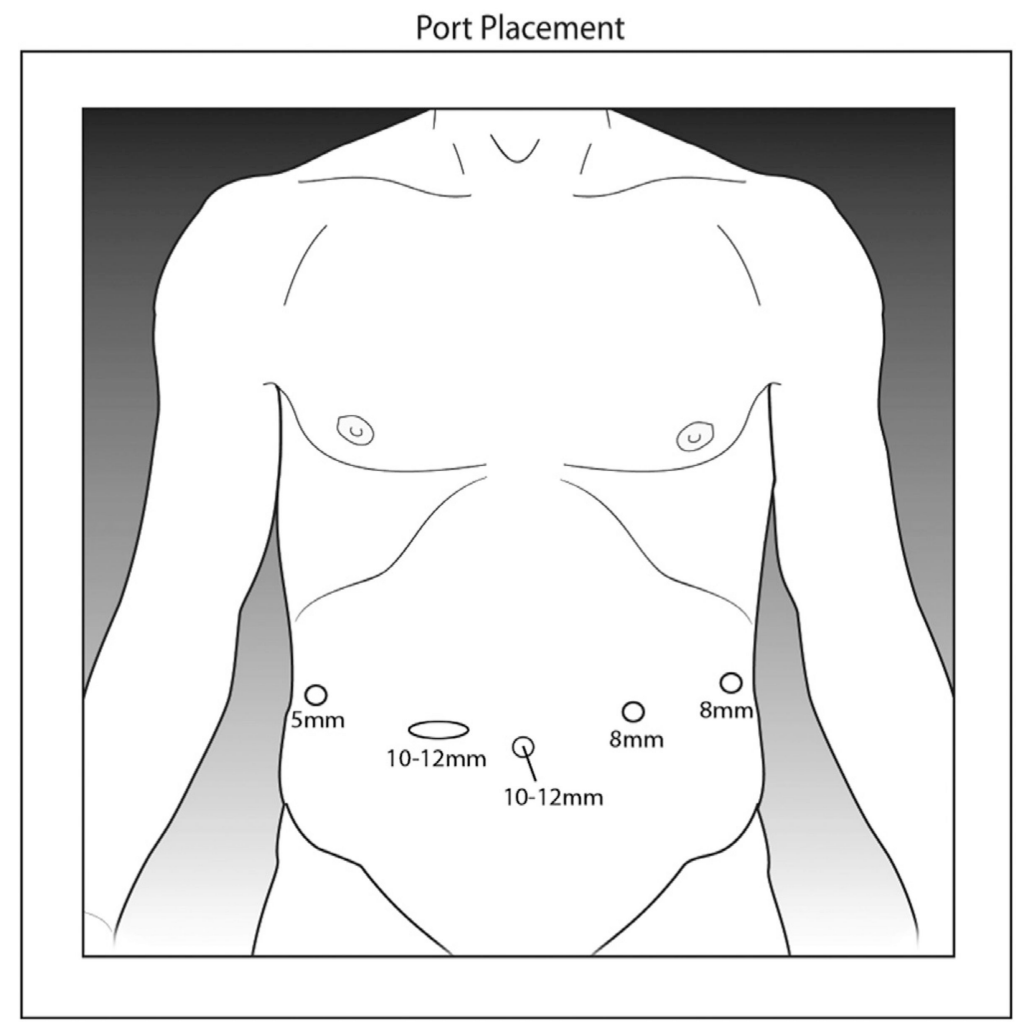

Fig. 21.

Recommended port placement for robotic gastrectomy. 


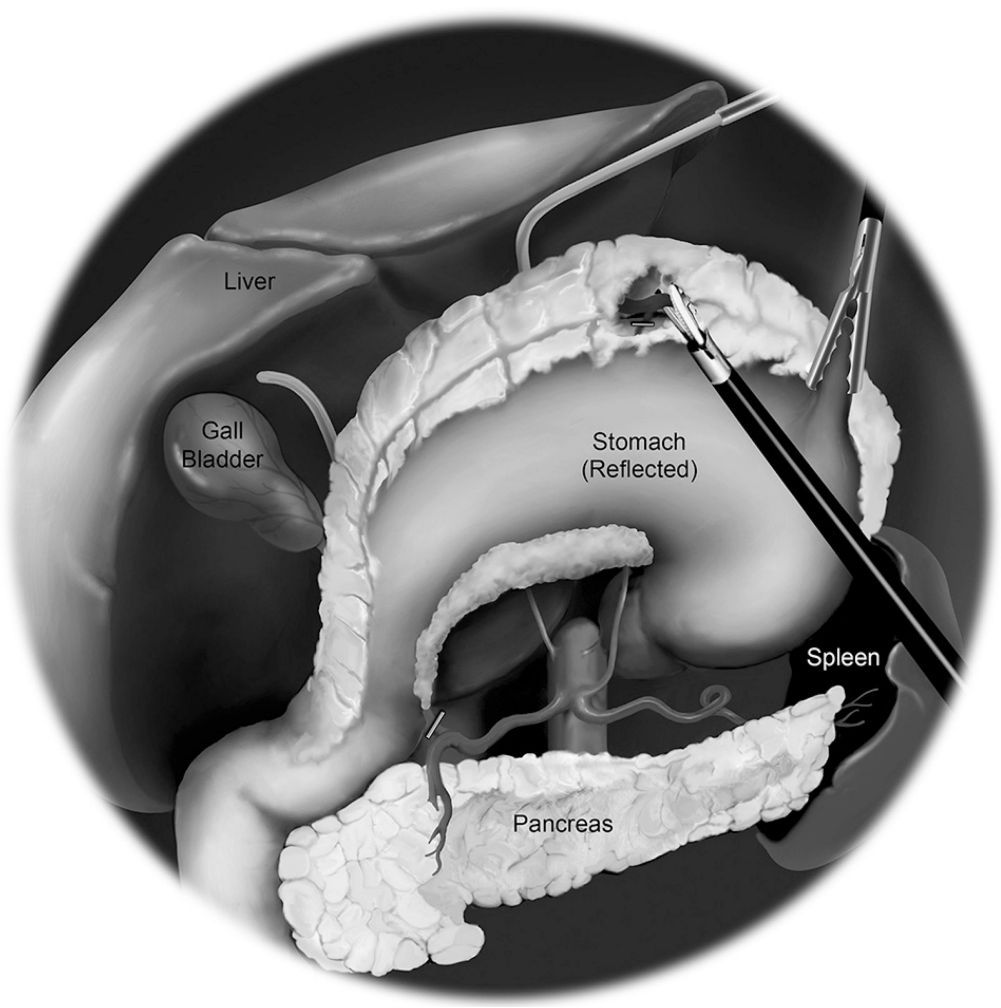

Fig. 22.

Entering the lesser sac and visualizing the posterior wall of the stomach allows the omentectomy to proceed safely to the splenic flexure. 


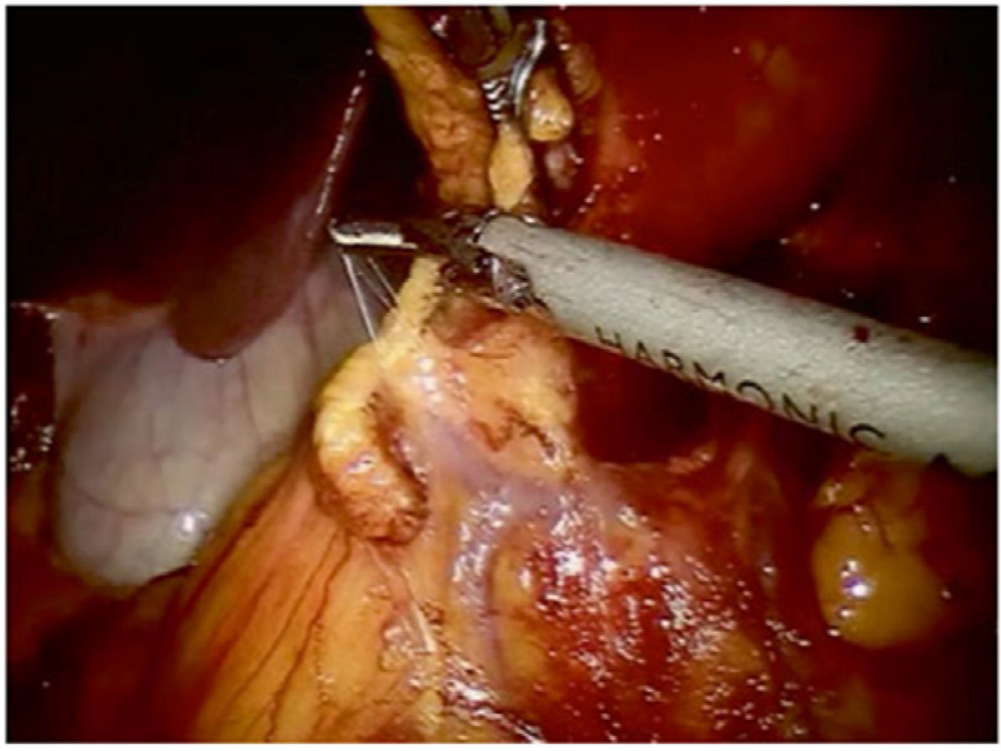

Fig. 23.

Confluence of right gastroepiploic and right colic veins at anterior border of pancreas. 


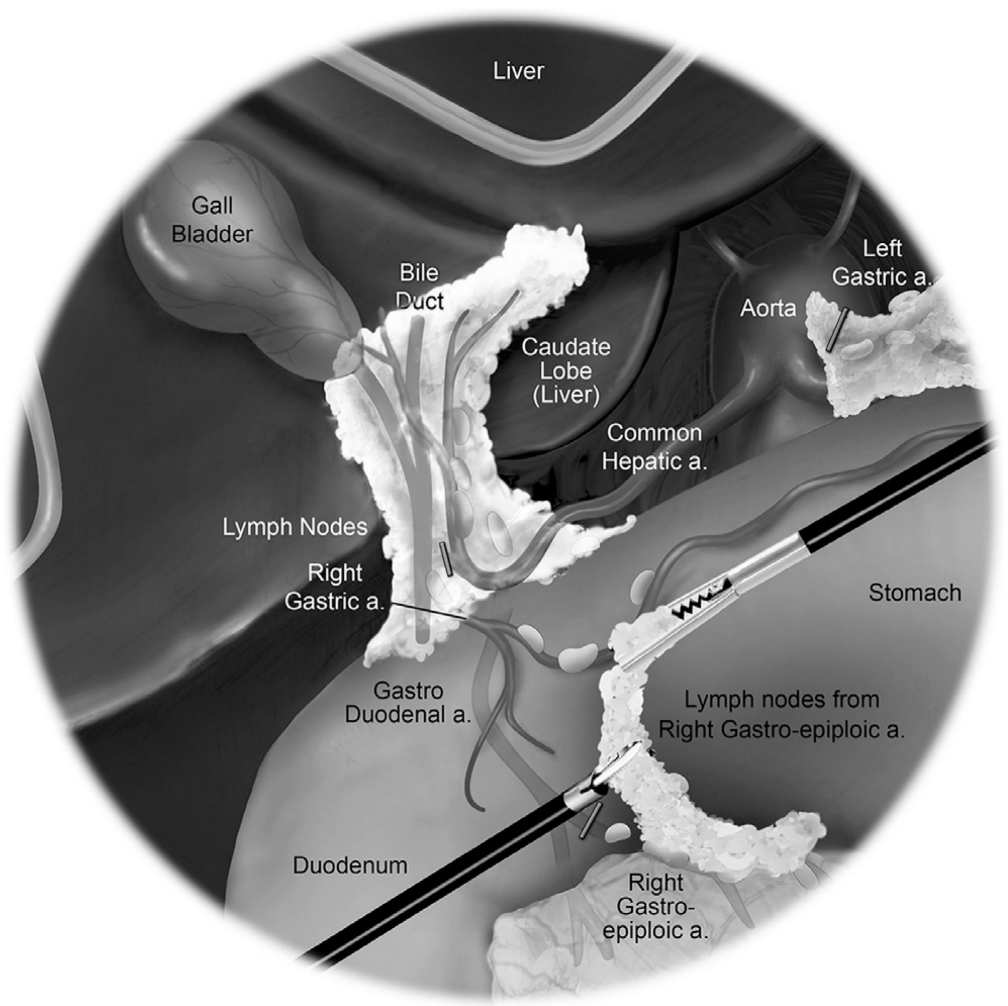

Fig. 24.

View of the proximal duodenum during dissection of the lymph nodes from the right gastroepiploic artery. 


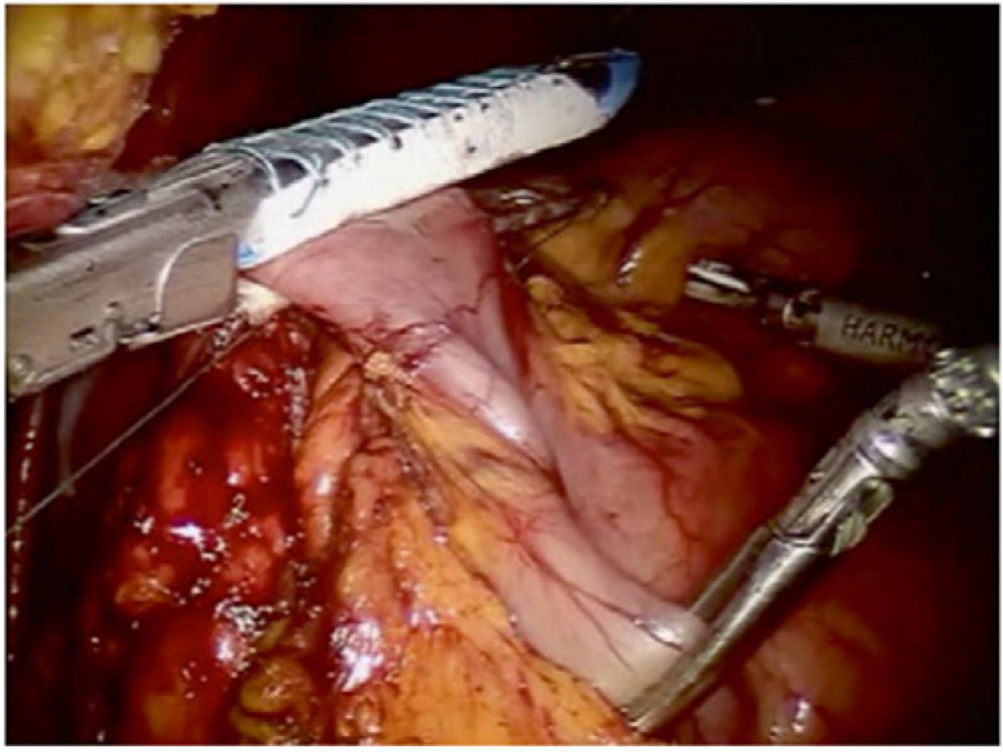

Fig. 25.

Division of proximal duodenum just distal to the pylorus. 

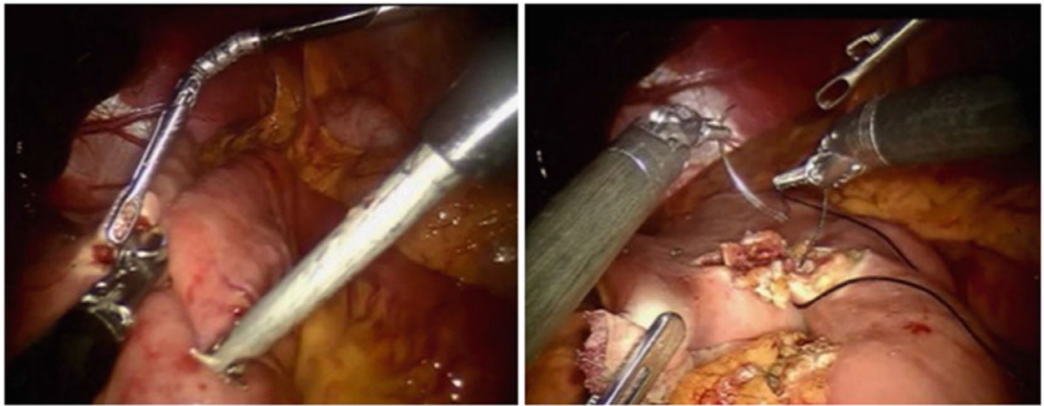

Fig. 26.

(a) Creation of stapled side-to-side gastrojejunostomy. (b) Closure of gastroenterotomy. 


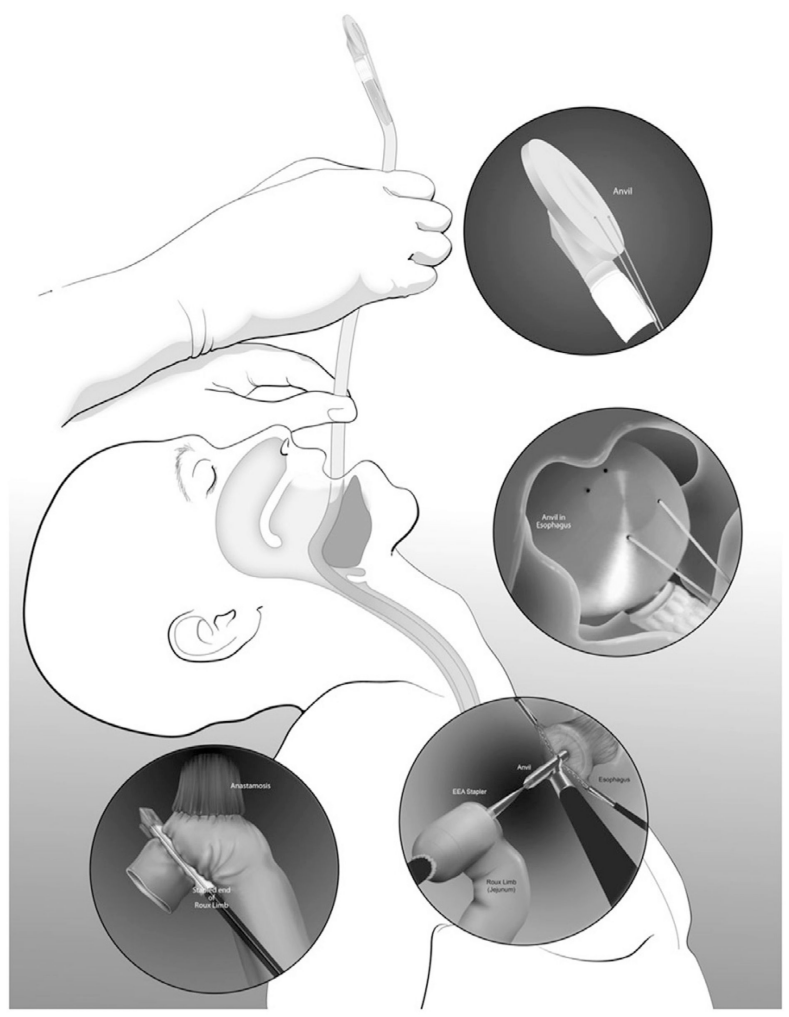

Fig. 27.

Use of a transoral anvil for construction of the esophagojejunostomy during total gastrectomy. 


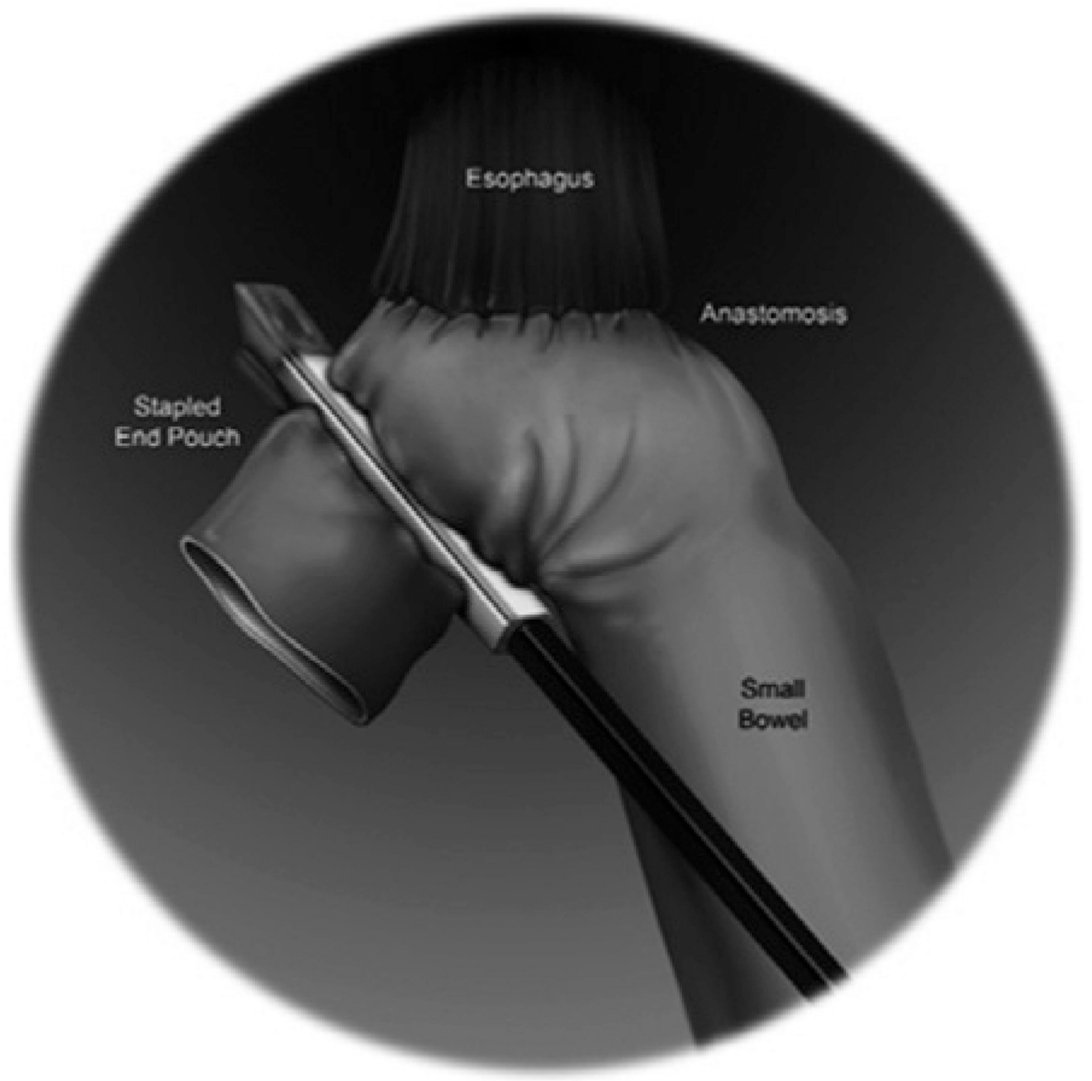

Fig. 28.

Schematic diagram of stapled esophagojejunostomy following total gastrectomy. 


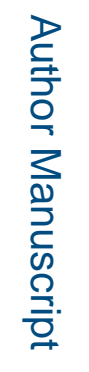

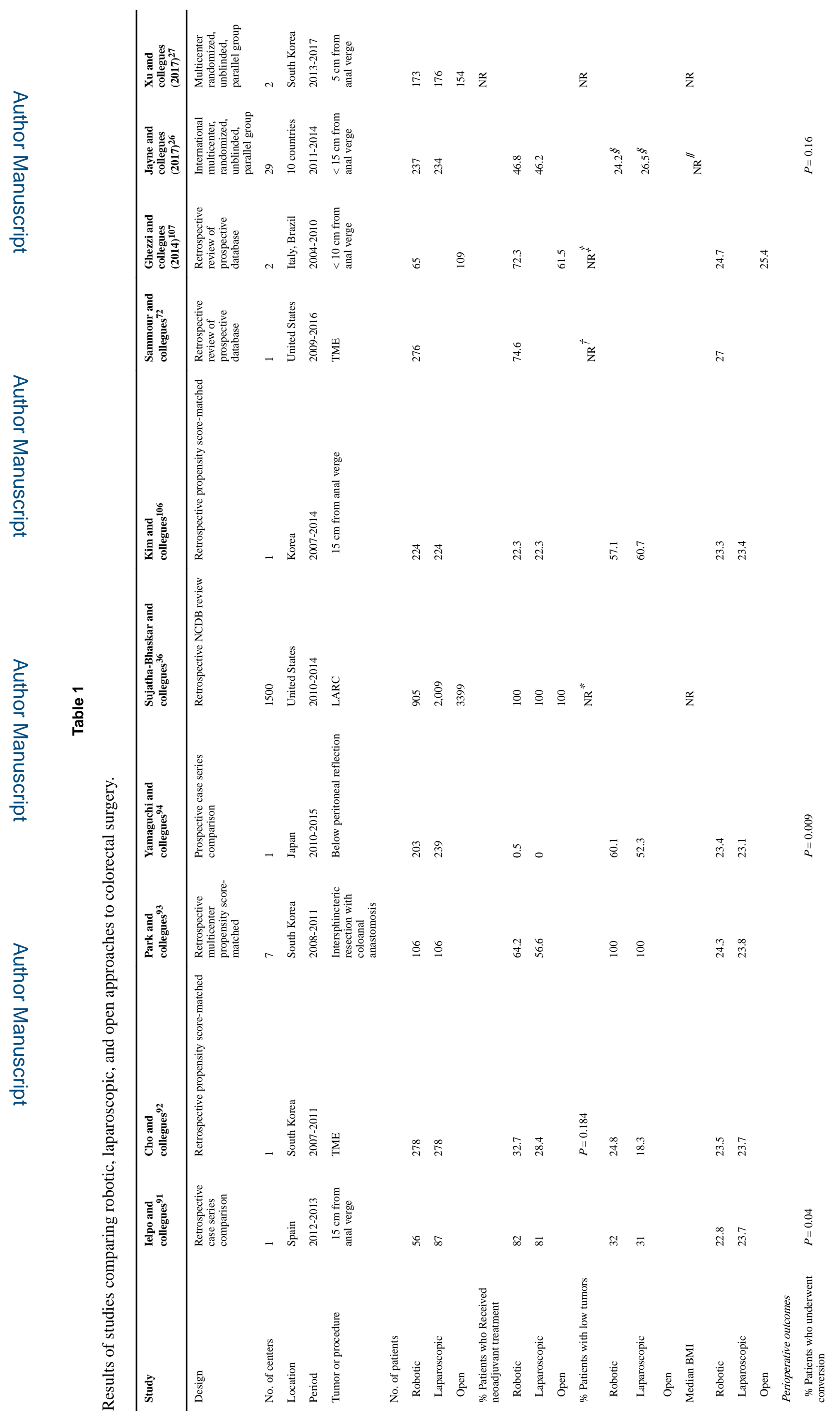




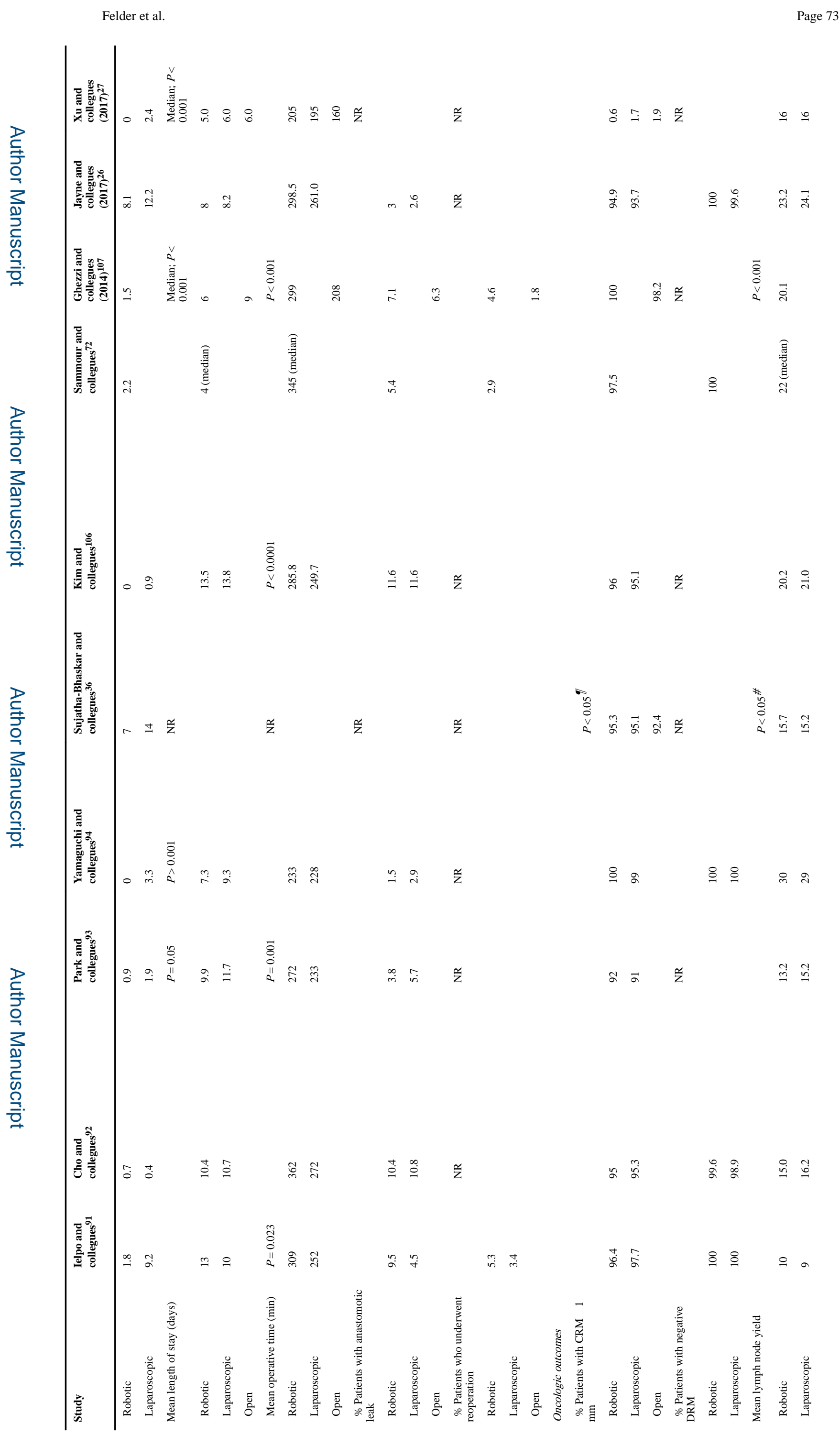

Curt Probl Surg. Author manuscript; available in PMC 2019 August 08. 


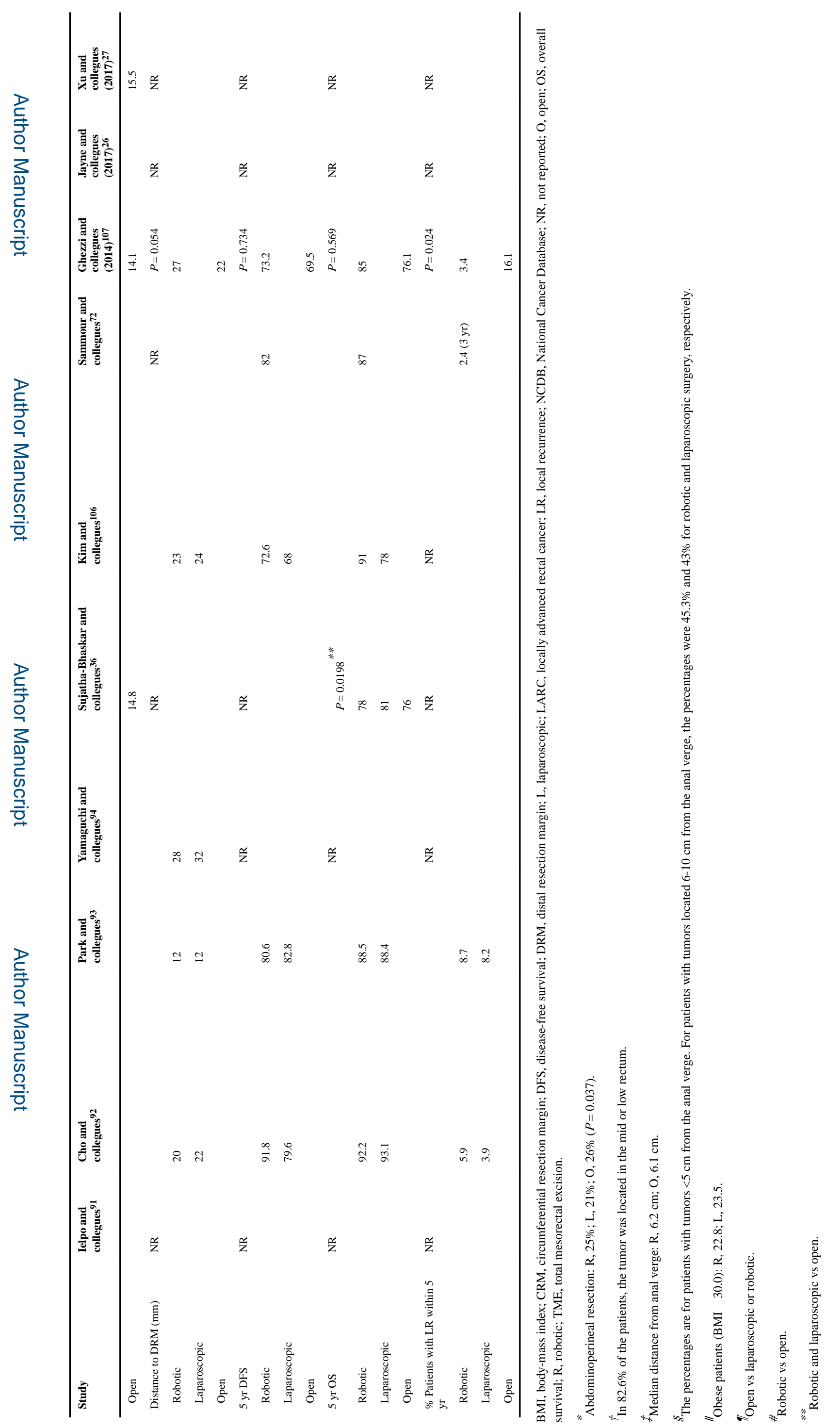




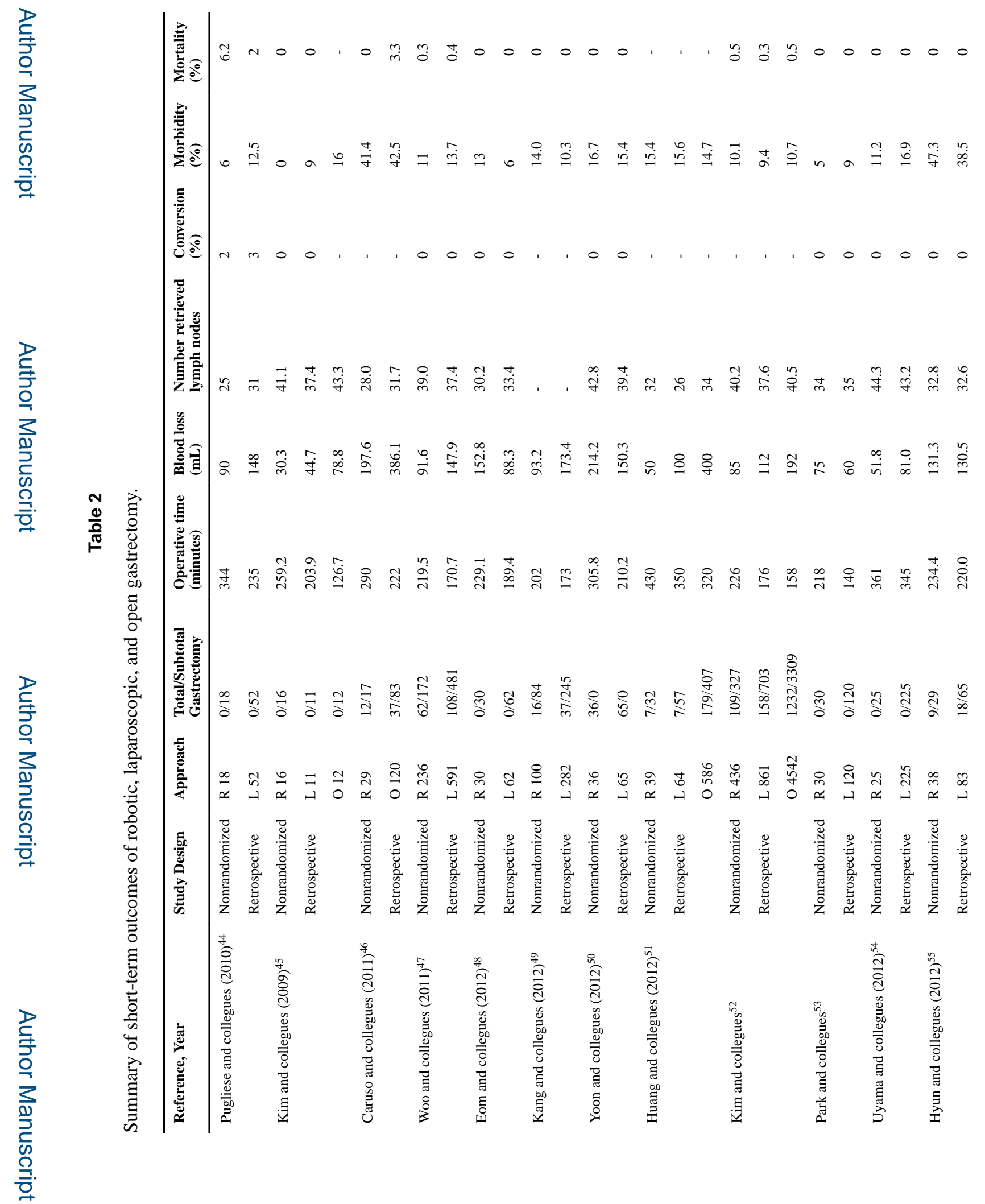

Curr Probl Surg. Author manuscript; available in PMC 2019 August 08. 
Felder et al.

Page 76

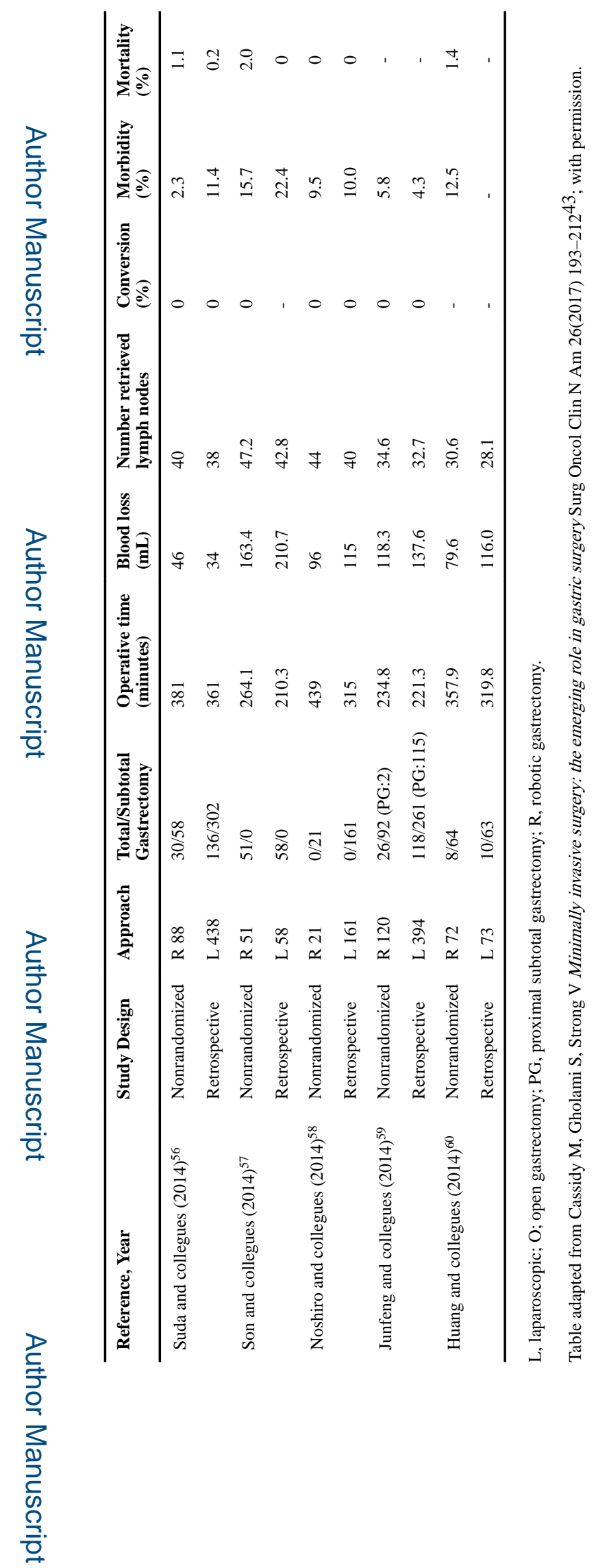

Curr Probl Surg. Author manuscript; available in PMC 2019 August 08. 